\title{
A STRATONOVICH-SKOROHOD INTEGRAL FORMULA FOR GAUSSIAN ROUGH PATHS
}

\author{
By ThOMAS CASS ${ }^{1}$ AND NENGLi LiM ${ }^{2}$ \\ Imperial College London and Singapore University of Technology and Design
}

Given a Gaussian process $X$, its canonical geometric rough path lift $\mathbf{X}$, and a solution $Y$ to the rough differential equation (RDE) $\mathrm{d} Y_{t}=V\left(Y_{t}\right) \circ \mathrm{d} \mathbf{X}_{t}$, we present a closed-form correction formula for $\int Y \circ \mathrm{d} \mathbf{X}-\int Y \mathrm{~d} X$, that is, the difference between the rough and Skorohod integrals of $Y$ with respect to $X$. When $X$ is standard Brownian motion, we recover the classical Stratonovichto-Itô conversion formula, which we generalize to Gaussian rough paths with finite $p$-variation, $p<3$, and satisfying an additional natural condition. This encompasses many familiar examples, including fractional Brownian motion with $H>\frac{1}{3}$. To prove the formula, we first show that the Riemann-sum approximants of the Skorohod integral converge in $L^{2}(\Omega)$ by using a novel characterization of the Cameron-Martin norm in terms of higher-dimensional Young-Stieltjes integrals. Next, we append the approximants of the Skorohod integral with a suitable compensation term without altering the limit, and the formula is finally obtained after a rebalancing of terms.

\section{CONTENTS}

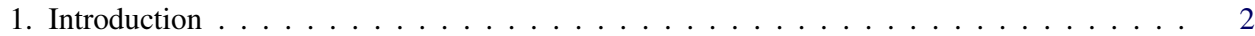

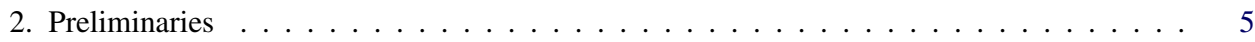

2.1. Rough paths, $p$-variation topology and controls $\ldots \ldots \ldots \ldots \ldots \ldots$

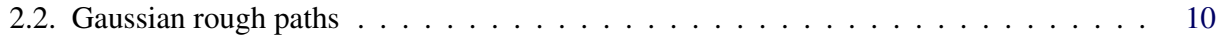

2.3. Malliavin calculus . . . . . . . . . . . . . . . . . . . . 13

2.4. Rough integration and controlled rough paths . . . . . . . . . . . . . . 16

2.5. Rough differential equations . . . . . . . . . . . . . . . . . . 17

3. High-order directional derivatives for solutions to RDEs _ . . . . . . . . . . . 20

3.1. Upper bounds on the high-order directional derivatives . . . . . . . . . . . . . 22

4. An isomorphism and dense subspace of the Cameron-Martin space . . . . . . . . . 28

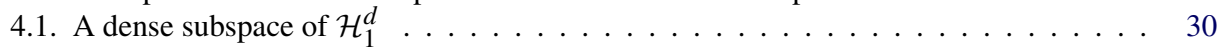

4.2. The Malliavin derivative and convergence in the tensor norm . . . . . . . . . . 31

4.3. The Itô-Skorohod isometry revisited . . . . . . . . . . . . . . . . . . . . 37

4.4. Riemann sum approximation to the Skorohod integral . . . . . . . . . . . . . . . 40

5. Appending the Riemann sum approximation to the Skorohod integral . . . . . . . . . . . 42

Received May 2016; revised January 2018.

${ }^{1}$ Supported by EPSRC Grant EP/M00516X/1.

${ }^{2}$ Part of the work was carried out while the second author was a PhD candidate at ICL, supported by the Roth scholarship and the Imperial College-NUS joint $\mathrm{PhD}$ scholarship.

MSC2010 subject classifications. Primary 60H05; secondary 60H07, 60H10.

Key words and phrases. Rough paths theory, Malliavin calculus, generalized Itô-Stratonovich correction formulas. 
6. Conversion formula . . . . . . . . . . . . . . . . . . . 50

6.1. Application of the correction formula to fractional Brownian motion . . . . . . . . 54

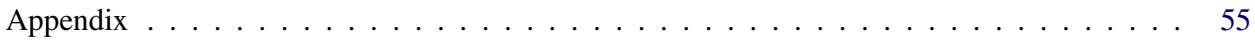

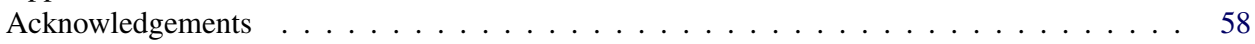

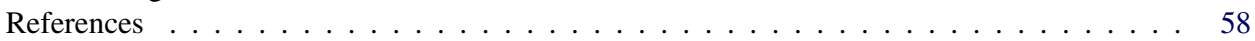

1. Introduction. Gaussian processes are used in modeling natural phenomena, from rough stochastic volatility models in high-frequency trading [2], to models of vortex filaments based on fractional Brownian motion [31]. To analyze stochastic processes with regularity lower than standard Brownian motion, one can employ the theory of rough paths [27]. In particular, given a Gaussian process $X$, one can lift it canonically to a geometric rough path $\mathbf{X}$ [17], and this allows one to study the properties of rough differential equations (RDEs)

$$
\mathrm{d} Y_{t}=V\left(Y_{t}\right) \circ \mathrm{d} \mathbf{X}_{t}, \quad Y_{0}=y_{0} \in \mathbb{R}^{e},
$$

and of rough integrals of the form

$$
\int_{0}^{T} Y_{t} \circ \mathrm{d} \mathbf{X}_{t}
$$

Furthermore, this geometric calculus generalizes Stratonovich's stochastic calculus, and as such, it finds natural applications, for example, in stochastic geometry where the change-of-variable formula allows one to give an intrinsic and coordinate-invariant definition of a rough path on a general smooth manifold; cf. $[5,9]$.

Itô integrals, by contrast, preserve the local martingale property, which is a useful feature when computing probabilistic quantities such as exit distributions and conditional expectations. One can often gain insight into a problem by transforming Stratonovich integrals to Itô integrals and vice versa, depending on the requirement at hand.

Now if $Y$ and $X$ are two continuous semimartingales, both $\mathbb{R}^{d}$-valued, it is well known that the difference between the two integrals is given in terms of the quadratic covariation through the formula (cf. [24, 33])

$$
\int_{0}^{T}\left\langle Y_{t}, \mathrm{od} X_{t}\right\rangle=\int_{0}^{T}\left\langle Y_{t}, \mathrm{~d} X_{t}\right\rangle+\frac{1}{2}[Y, X]_{T} .
$$

In the case where $Y_{t}$ solves RDE (1) and $X_{t}$ is taken to be standard Brownian motion $B_{t}$, this becomes

$$
\int_{0}^{T}\left\langle Y_{t}, \circ \mathrm{d} B_{t}\right\rangle=\int_{0}^{T}\left\langle Y_{t}, \mathrm{~d} B_{t}\right\rangle+\frac{1}{2} \int_{0}^{T} \operatorname{tr}\left[V\left(Y_{t}\right)\right] \mathrm{d} t,
$$

where in the second term on the right-hand side we have the usual trace of $V\left(Y_{t}\right) \in$ $\mathbb{R}^{d} \otimes \mathbb{R}^{d}$ considered as a $d$-by- $d$ matrix. 
On the other hand, if $Y_{t} \equiv \nabla f\left(X_{t}\right)$, where $f$ is sufficiently smooth, then we get Itô's formula,

$$
\begin{aligned}
f\left(X_{T}\right)-f\left(X_{0}\right) & =\int_{0}^{T}\left\langle\nabla f\left(X_{t}\right), \mathrm{od} \mathbf{X}_{t}\right\rangle \\
& =\int_{0}^{T}\left\langle\nabla f\left(X_{t}\right), \mathrm{d} X_{t}\right\rangle+\frac{1}{2} \int_{0}^{T} \Delta f\left(X_{t}\right) \mathrm{d} R(t),
\end{aligned}
$$

where the first term on the right-hand side is the Skorohod integral of $\nabla f(X)$ with respect to $X$, and $R(t)$ is the variance $\mathbb{E}\left[\left(X_{t}^{(1)}\right)^{2}\right]$. This has been well studied for general Gaussian processes, particularly fractional Brownian motion, over the past two decades; see [1, 4, 30, 32], and in particular [22], which uses rough path techniques to prove the formula.

Our main result is the following theorem, where the driving signal $\mathbf{X}$ is constructed from the limit of the piecewise-linear approximations of a Gaussian process with i.i.d. components.

TheOREM. For $2 \leq p<3$, let $Y \in \mathcal{C}^{p \text {-var }}\left([0, T] ; \mathbb{R}^{d}\right)$ denote the path-level solution to

$$
\mathrm{d} Y_{t}=V\left(Y_{t}\right) \circ \mathrm{d} \mathbf{X}_{t}, \quad Y_{0}=y_{0},
$$

where $V \in \mathcal{C}_{b}^{6}\left(\mathbb{R}^{d} ; \mathbb{R}^{d} \otimes \mathbb{R}^{d}\right)$ and $\mathbf{X} \in \mathcal{C}^{0, p-\operatorname{var}}\left([0, T] ; G{ }^{\lfloor p\rfloor}\left(\mathbb{R}^{d}\right)\right)$. We assume the covariance function $R$ of $\mathbf{X}$ is of finite $2 D \rho$-variation, $1 \leq \rho<\frac{3}{2}$ and satisfies

$$
\|R(t, \cdot)-R(s, \cdot)\|_{\rho-\operatorname{var} ;[0, T]} \leq C|t-s|^{\frac{1}{\rho}}
$$

for all $s, t \in[0, T]$. Then almost surely, we have

$$
\begin{aligned}
\int_{0}^{T}\left\langle Y_{t}, \circ \mathrm{d} \mathbf{X}_{t}\right\rangle= & \int_{0}^{T}\left\langle Y_{t}, \mathrm{~d} X_{t}\right\rangle+\frac{1}{2} \int_{0}^{T} \operatorname{tr}\left[V\left(Y_{t}\right)\right] \mathrm{d} R(t) \\
& +\int_{[0, T]^{2}} \mathbb{1}_{[0, t)}(s) \operatorname{tr}\left[J_{t}^{\mathbf{X}}\left(J_{s}^{\mathbf{X}}\right)^{-1} V\left(Y_{s}\right)-V\left(Y_{t}\right)\right] \mathrm{d} R(s, t) .
\end{aligned}
$$

Here, $J_{t}^{\mathbf{X}}$ denotes the Jacobian of the flow map $y_{0} \rightarrow Y_{t}$. The last term on the right-hand side of (7) is a proper 2D Young-Stieltjes integral with respect to the covariance function. When $X$ is standard Brownian motion, it vanishes since the integrand is zero on the diagonal and $\mathrm{d} R(s, t)=\delta_{\{s=t\}} \mathrm{d} s \mathrm{~d} t$. This, together with the fact that $R(t)=t$, allows us to recover the usual Itô-Stratonovich conversion formula (3).

Hence, an immediate contribution of the theorem is the generalization of formula (3) to the setting where the integrands are solutions to Gaussian RDEs. Here, we are able to give a formula for integrators other than standard Brownian motion without restriction on the regularity of the integrand; compare this to [12] 
for example, where essentially Young complementary regularity is required. Furthermore, the novel 2D Young-Stieltjes integral can be understood as measuring the failure of the commutativity of $V$ with respect to the covariance of the Gaussian process. For studying the dynamics of Gaussian processes in cases where the correction formula is indispensable, for example, Gaussian processes evolving on manifolds, this could lead to new insights.

We now provide the main idea behind the proof of the theorem. Denoting $\mathbf{X}=$ $\left(1, X, \mathbf{X}^{2}\right)$, the solution $Y$ to RDE (1) can be integrated against this rough path and

$$
\int_{0}^{T}\left\langle Y_{t}, \mathrm{od} \mathbf{X}_{t}\right\rangle=\lim _{\|\pi\| \rightarrow 0} \sum_{i}\left\langle Y_{t_{i}}, X_{t_{i}, t_{i+1}}\right\rangle+V\left(Y_{t_{i}}\right)\left(\mathbf{X}_{t_{i}, t_{i+1}}^{2}\right)
$$

almost surely. Continuing, we devote Section 4 to proving two claims. The first is that $Y$ lies in the domain of the Skorohod integral operator w.r.t. $X$, and the second is that, as a limit in $L^{2}(\Omega)$ we have

$$
\begin{aligned}
& \int_{0}^{T}\left\langle Y_{t}, \mathrm{~d} X_{t}\right\rangle \\
& \quad=\lim _{\|\pi\| \rightarrow 0} \sum_{i}\left[\left\langle Y_{t_{i}}, X_{t_{i}, t_{i+1}}\right\rangle-\int_{0}^{t_{i}} \operatorname{tr}\left[J_{t_{i}}^{\mathbf{X}}\left(J_{s}^{\mathbf{X}}\right)^{-1} V\left(Y_{s}\right)\right] R\left(\Delta_{i}, \mathrm{~d} s\right)\right] .
\end{aligned}
$$

Proving these facts is less obvious than in the case where $Y_{t}=\nabla f\left(X_{t}\right)$, and we need to use the tail estimates of [10] in a fundamental way. In Section 5, we use condition (6) to prove that

$$
\sum_{i} V\left(Y_{t_{i}}\right)\left(\left(\mathbf{X}_{t_{i}, t_{i+1}}^{2}\right)-\frac{1}{2} \mathbb{E}\left[\left(X_{t_{i}, t_{i+1}}^{(1)}\right)^{2}\right] \mathcal{I}_{d}\right)
$$

has a vanishing $L^{2}(\Omega)$ limit as $\|\pi\| \rightarrow 0$. The proof of this relies on estimates coming from a delicate interplay between the theory of Malliavin calculus and Gaussian rough paths; see Proposition 5.1. After augmenting (10) to (9) and extracting an almost sure subsequence, we can take the difference between this subsequence and (8). A careful rearrangement of the terms in this difference will then yield the correction term.

We now outline the structure of the paper, as well as highlight other contributions that are of independent interest. We begin in Section 2 with a summary of Gaussian rough path concepts and a primer on the Malliavin calculus as applied to RDE solutions. In Section 3.1, we provide a general closed-form expression and a novel bound for the higher-order Malliavin derivatives of RDE solutions relative to the driving rough path (cf. $[8,21,23])$. This will be then used in Section 5 to show that (10) has vanishing $L^{2}(\Omega)$ limit.

In Section 4, we give a new characterization of the Cameron-Martin norm in terms of multi-dimensional Young-Stieltjes integrals. We show that one can identify $\mathcal{C}^{p \text {-var }}([0, T])$ with a dense subspace of $\mathcal{H}_{1}$, the Hilbert space generated by the 
indicator functions which is isomorphic to the Cameron-Martin space. In particular, for $f \in \mathcal{C}^{p \text {-var }}([0, T])$, we have

$$
\|f\|_{\mathcal{H}_{1}}=\sqrt{\int_{[0, T]^{2}} f_{t} f_{s} \mathrm{~d} R(s, t) .}
$$

We also give a corresponding characterization with regards to the tensor product of the Cameron-Martin space in Section 4.2, and revisit the classical ItôSkorohod isometry in Section 4.3 by giving it a new formulation in terms of multidimensional Young-Stieltjes integrals. Finally, Section 4 is concluded with a proof of (9).

The main theorem and its proof is given in Section 6.

2. Preliminaries. The following is a summary of basic notation that will be used throughout the paper.

We will use $\left\{e_{j}\right\}, j=1, \ldots, d$, to denote the canonical basis for $\mathbb{R}^{d}$, and $|\cdot|$ will denote the standard Euclidean norm.

$\wedge$ and $\vee$ will be used to denote the min and max operators, respectively, and $C$, with or without subscript, will always denote a finite constant which may vary from line to line.

The notation $\mathcal{C}_{b}^{n}$ will be used when denoting a class of functions which have bounded derivatives up to $n$th order.

Given vector spaces $A$ and $B, \mathcal{L}(A ; B)$ will denote the space of linear maps from $A$ to $B$.

Frequently, we will canonically identify a tensors $\sum_{i=1}^{k} \sum_{j=1}^{l} a_{i j} e_{i} \otimes e_{j}$ (or cotensors $\left.\sum_{i=1}^{k} \sum_{j=1}^{l} a_{i j} \mathrm{~d} e_{i} \otimes \mathrm{d} e_{j}\right)$ in $\mathbb{R}^{k} \otimes \mathbb{R}^{l}$ with a $k$-by- $l$ matrix.

For simplification, we will denote both tensor spaces and co-tensor spaces with $\mathbb{R}^{k} \otimes \mathbb{R}^{l}$, and if $A \in \mathbb{R}^{k} \otimes \mathbb{R}^{k}, \operatorname{tr} A:=\sum_{i=1}^{k} a_{i i}$ will denote the usual trace operation.

$\mathcal{I}_{k}:=\sum_{j=1}^{k} e_{j} \otimes e_{j}$ will be used to denote the $k$-by- $k$ identity matrix.

2.1. Rough paths, p-variation topology and controls. We begin by reviewing the basic concepts and notation of rough paths theory. The standard references in this area include $[14,25,26]$ and [18].

Given $x \in \mathcal{C}\left([0, T] ; \mathbb{R}^{d}\right)$, that is, a continuous $\mathbb{R}^{d}$-valued path defined on the time interval $[0, T]$, where $T$ is some arbitrary but fixed terminal time, a rough path $\mathbf{x}$ includes the higher-order iterated integrals of $x$ in addition to the first-order increment $x_{s, t}:=x_{t}-x_{s}$. To account for these higher-order increments, the right space for $\mathbf{x}$ to take values in turns out to be the step- $n$ nilpotent group, which we will define below.

First, let $T^{n}\left(\mathbb{R}^{d}\right)$ denote the truncated tensor algebra up to degree $n$ :

$$
T^{n}\left(\mathbb{R}^{d}\right):=\mathbb{R} \oplus \mathbb{R}^{d} \oplus \cdots \oplus\left(\mathbb{R}^{d}\right)^{\otimes n}
$$


Addition and scalar multiplication are defined in the usual fashion, and given $a=$ $\left(a^{0}, a^{1}, \ldots, a^{n}\right), b=\left(b^{0}, b^{1}, \ldots, b^{n}\right) \in T^{n}\left(\mathbb{R}^{d}\right)$, multiplication is performed by

$$
a \otimes b:=\left(c^{0}, c^{1}, \ldots, c^{n}\right), \quad c^{k}=\sum_{i=0}^{k} a^{i} \otimes b^{k-i}, \forall 0 \leq k \leq n,
$$

where here we abuse the notation by reusing the same symbol for the tensor product in $\mathbb{R}^{d}$.

The tangent space of $T^{n}\left(\mathbb{R}^{d}\right)$ at the unit element $e=(1,0, \ldots, 0)$ is given by

$$
A_{T}^{n}\left(\mathbb{R}^{d}\right):=0 \oplus \mathbb{R}^{d} \oplus \cdots \oplus\left(\mathbb{R}^{d}\right)^{\otimes n} .
$$

We will define the exponential map exp : $A_{T}^{n}\left(\mathbb{R}^{d}\right) \rightarrow T^{n}\left(\mathbb{R}^{d}\right)$ by

$$
\exp (a):=\sum_{i=0}^{n} \frac{a^{\otimes i}}{i !}
$$

[for $a \in \mathbb{R}^{d}$ we will occasionally abuse the notation by denoting $\exp (a):=$ $\exp ((0, a, 0))]$, and the logarithm map $\log : T^{n}\left(\mathbb{R}^{d}\right) \rightarrow A_{T}^{n}\left(\mathbb{R}^{d}\right)$ by

$$
\log (a)=\sum_{i=1}^{n}(-1)^{i+1} \frac{(a-e)^{\otimes i}}{i} .
$$

DEFinition 2.1. The step- $n$ nilpotent group (with $d$ generators), denoted by $G^{n}\left(\mathbb{R}^{d}\right)$, is the subgroup of $T^{n}\left(\mathbb{R}^{d}\right)$ corresponding to the sub-Lie algebra of $A_{T}^{n}\left(\mathbb{R}^{d}\right)$ generated by the Lie bracket $[a, b]=a \otimes b-b \otimes a$.

One can check that the inverse of any element $a=e+\tilde{a} \in G^{n}\left(\mathbb{R}^{d}\right)$ is given by

$$
a^{-1}=\sum_{k=0}^{n}(-1)^{k} \tilde{a}^{\otimes k}
$$

see Lemma 7.16 in [18].

$G^{n}\left(\mathbb{R}^{d}\right)$ will be equipped with the topology induced from the symmetric, subadditive homogeneous norm

$$
\|g\|=\max _{i=1, \ldots, n}\left(i !\left|g^{i}\right|\right)^{\frac{1}{i}} .
$$

Consider now $\mathbf{x} \in \mathcal{C}\left([0, T] ; G^{n}\left(\mathbb{R}^{d}\right)\right)$, a continuous $G^{n}\left(\mathbb{R}^{d}\right)$ valued path on $[0, T]$. We define the increment by setting $\mathbf{x}_{s, t}:=\mathbf{x}_{s}^{-1} \otimes \mathbf{x}_{t}$. Such a path is called a multiplicative functional (cf. [27]) as it satisfies Chen's equality

$$
\mathbf{x}_{s, t}=\mathbf{x}_{s, u} \otimes \mathbf{x}_{u, t} \quad \forall s, u, t \in[0, T], s \leq u \leq t .
$$

We now define the $p$-variation distance as

$$
d_{p-\operatorname{var} ;[0, T]}(\mathbf{x}, \mathbf{y}):=\sup _{\pi}\left(\sum_{i} d\left(\mathbf{x}_{t_{i}, t_{i+1}}, \mathbf{y}_{t_{i}, t_{i+1}}\right)^{p}\right)^{\frac{1}{p}},
$$


where the supremum runs over all partitions $\pi=\left\{t_{i}\right\}$ of $[0, T]$. We also define

$$
\|\mathbf{x}\|_{p \text {-var; }[0, T]}:=d_{p \text {-var; }[0, T]}(\mathbf{x}, 0),
$$

where 0 denotes the constant path $\mathbf{y}_{t}=e$ for all $t \in[0, T]$.

DEFINITION 2.2. For $p \geq 1$, the weakly geometric $p$-rough paths, which we will denote by $\mathcal{C}^{p \text {-var }}\left([0, T] ; G^{\lfloor p\rfloor}\left(\mathbb{R}^{d}\right)\right)$, is the set of continuous functions $\mathbf{x}$ from $[0, T]$ onto $G^{\lfloor p\rfloor}\left(\mathbb{R}^{d}\right)$ such that $\|\mathbf{x}\|_{p \text {-var; }[0, T]}<\infty$.

The simplest example of a weakly geometric $p$-rough path is as follows, given a bounded-variation path $x$ in $\mathbb{R}^{d}$, we can compute the signature of $x$ in $G^{\lfloor p\rfloor}\left(\mathbb{R}^{d}\right)$ :

$$
S_{\lfloor p\rfloor}(x)_{s, t}=\left(1, \mathbf{x}_{s, t}^{1}, \mathbf{x}_{s, t}^{2}, \ldots, \mathbf{x}_{s, t}^{\lfloor p\rfloor}\right),
$$

where $\mathbf{x}_{s, t}^{k}$ is the conventional $k$ th iterated integral of the path $x$ over the interval $[s, t]$ :

$$
\mathbf{x}_{s, t}^{k}=\sum_{j_{1}, \ldots, j_{k}=1}^{d}\left(\int_{s<r_{1}<\cdots<r_{k}<t} \mathrm{~d} x_{r_{1}}^{\left(j_{1}\right)} \otimes \cdots \otimes \mathrm{d} x_{r_{k}}^{\left(j_{k}\right)}\right) e_{j_{1}} \otimes \cdots \otimes e_{j_{k}} .
$$

Let $\mathcal{C}^{\infty}\left([0, T] ; G^{\lfloor p\rfloor}\left(\mathbb{R}^{d}\right)\right)$ denote the subset of weakly-geometric rough paths which are also of bounded variation. Then the signature of $x$ is in $\mathcal{C}^{\infty}([0, T]$; $G^{\lfloor p\rfloor}\left(\mathbb{R}^{d}\right)$ ), and we also have the following definition.

DEFINITION 2.3. For $p \geq 1$, the space of geometric p-rough paths, which we will denote by $\mathcal{C}^{0, p \text {-var }}\left([0, T] ; G^{\lfloor p\rfloor}\left(\mathbb{R}^{d}\right)\right)$, is defined to be the closure of $\mathcal{C}^{\infty}\left([0, T] ; G^{\lfloor p\rfloor}\left(\mathbb{R}^{d}\right)\right)$ in $\mathcal{C}^{p \text {-var }}\left([0, T] ; G^{\lfloor p\rfloor}\left(\mathbb{R}^{d}\right)\right)$ with respect to the topology given by the $p$-variation distance (17).

REMARK 2.4. In finite dimensions, the difference between weakly-geometric rough paths and geometric rough paths is fairly minor, as we have the following relation:

$$
\begin{aligned}
\mathcal{C}^{0, p-\operatorname{var}}\left([0, T] ; G^{\lfloor p\rfloor}\left(\mathbb{R}^{d}\right)\right) & \subset \mathcal{C}^{p-\operatorname{var}}\left([0, T] ; G^{\lfloor p\rfloor}\left(\mathbb{R}^{d}\right)\right) \\
& \subset \mathcal{C}^{0, p+\varepsilon-\operatorname{var}}\left([0, T] ; G^{\lfloor p\rfloor}\left(\mathbb{R}^{d}\right)\right),
\end{aligned}
$$

where $\varepsilon>0$ can be chosen arbitrarily small; cf. [16].

We will now extend the notion of finite $p$-variation to general metric spaces. Given a metric space $(E, d)$, a path $f:[0, T] \rightarrow E$ is said to have finite $p$-variation if

$$
\|f\|_{p-\operatorname{var} ;[s, t]}:=\sup _{\pi}\left(\sum_{i} d\left(f_{t_{i}}, f_{t_{i+1}}\right)_{E}^{p}\right)^{\frac{1}{p}}<\infty .
$$


We will use $V^{p \text {-var }}([0, T] ; E)$ to denote the space of functions which satisfy the bound above, and $\mathcal{C}^{p-\operatorname{var}}([0, T] ; E)$ to denote the set of continuous functions which satisfy (18).

We will also define the notation $\mathcal{C}_{\mathrm{pw}}^{p \text {-var }}([0, T] ; E)$ for piecewise-continuous functions of bounded $p$-variation as follows: $f$ is in $\mathcal{C}_{\mathrm{pw}}^{p \text {-var }}([0, T] ; E)$ if there exists a partition $\left\{t_{i}\right\}$ of $[0, T]$ such that $f$ is in $\mathcal{C}^{p \text {-var }}\left(\left(t_{i}, t_{i+1}\right) ; E\right)$ for all $i$.

We have the following simple proposition; cf. Proposition 5.3 in [18].

Proposition 2.5. Let $f \in \mathcal{C}([0, T] ; E)$. Then if $1 \leq p \leq p^{\prime}<\infty$,

$$
\|f\|_{p^{\prime} \text {-var; }[0, T]} \leq\|f\|_{p \text {-var; }[0, T]} .
$$

In particular, $\mathcal{C}^{p-\operatorname{var}}([0, T] ; E) \subset \mathcal{C}^{p^{\prime}-\operatorname{var}}([0, T] ; E)$.

We will use the notation $\|f\|_{p \text {-var; }[s, t]}$ when the supremum is taken over partitions of $[s, t] \subset[0, T]$. Proposition 5.8 in [18] tells us that

$$
\omega(s, t):=\|f\|_{p \text {-var; }[s, t]}^{p}
$$

defines a control, that is, a continuous, nonnegative, real-valued function that is super-additive and vanishes on the diagonal, that is, $\omega(t, t)=0$ for all $t \in[0, T]$. We also note the following lemmas about controls.

LEMMA 2.6. Assume $\omega_{1}$ and $\omega_{2}$ are controls:

(i) If $\phi$ is a positive convex function, then $\phi\left(\omega_{1}\right)$ is a control.

(ii) Given $\alpha, \beta>0$ with $\alpha+\beta \geq 1, \omega_{1}^{\alpha} \omega_{2}^{\beta}$ is also a control.

PROOF. Exercises 1.8 and 1.9 in [18].

LEMMA 2.7 (Proposition 5.10 in [18]). Let $\omega$ be a control on $[0, T]$ and let $p \geq 1$. Then the pointwise estimate

$$
d\left(f_{s}, f_{t}\right)^{p} \leq C \omega(s, t) \quad \forall s<t \in[0, T]
$$

implies the p-variation estimate

$$
\|f\|_{p-\operatorname{var} ;[s, t]} \leq C^{\frac{1}{p}} \omega(s, t)^{\frac{1}{p}} \quad \forall s<t \in[0, T] .
$$

If $E$ also has a norm $\|\cdot\|_{E}$, we will also use the notation $\|f\|_{\mathcal{V}^{p} ;[0, T]}$ to denote the norm

$$
\begin{aligned}
\|f\|_{\mathcal{V}^{p} ;[0, T]} & :=\|f\|_{p-\operatorname{var} ;[0, T]}+\sup _{t \in[0, T]}\left\|f_{t}\right\|_{E} \\
& \leq\left\|f_{0}\right\|_{E}+2\|f\|_{p-\operatorname{var} ;[0, T]} .
\end{aligned}
$$


For a function defined on $[0, T]^{2}, f:[0, T]^{2} \rightarrow E$ is said to be of finite $2 \mathrm{D}$ $p$-variation if

$$
\|f\|_{p \text {-var; }[0, T]^{2}}:=\sup _{\pi}\left(\sum_{i, j}\left\|f\left(\begin{array}{c}
u_{i}, u_{i+1} \\
v_{j}, v_{j+1}
\end{array}\right)\right\|_{E}^{p}\right)^{\frac{1}{p}}<\infty,
$$

where $\pi=\left\{\left(u_{i}, v_{j}\right)\right\}$ is a partition of $[0, T]^{2}$, and the rectangular increment is given by

$$
f\left(\begin{array}{l}
u_{i}, u_{i+1} \\
v_{j}, v_{j+1}
\end{array}\right):=f\left(u_{i}, v_{j}\right)+f\left(u_{i+1}, v_{j+1}\right)-f\left(u_{i}, v_{j+1}\right)-f\left(u_{i+1}, v_{j}\right) .
$$

Similar to the $1 \mathrm{D}$ case, we will use $V^{p \text {-var }}\left([0, T]^{2} ; E\right)\left(\operatorname{resp} ., \mathcal{C}^{p \text {-var }}\left([0, T]^{2} ; E\right)\right)$ to denote the set of functions (resp., continuous functions) which satisfy (19).

On occasion, we will use the notation

$$
\begin{aligned}
& f\left(\Delta_{i}, v\right):=f\left(u_{i+1}, v\right)-f\left(u_{i}, v\right), \\
& f\left(u, \Delta_{j}\right):=f\left(u, v_{j+1}\right)-f\left(u, v_{j}\right) .
\end{aligned}
$$

Given a rectangle $R=[s, t] \times[u, v] \subset[0, T]^{2}$, we let $\Pi(R)$ denote the family of all partitions $\left\{\left[a_{i}, b_{i}\right] \times\left[c_{i}, d_{i}\right], i=1, \ldots, n\right\}$ of $R$, where in each partition, the subrectangles have disjoint interiors and satisfy $\bigcup_{i=1}^{n}\left[a_{i}, b_{i}\right] \times\left[c_{i}, d_{i}\right]=R$.

Definition 2.8. Let $\Delta_{T}:=\{(s, t) \mid 0 \leq s \leq t \leq T\}$. A map $\omega: \Delta_{T} \times \Delta_{T} \rightarrow$ $[0, \infty)$ is called a $2 \mathrm{D}$ control if it is continuous, zero on degenerate rectangles, and super-additive in the sense that for all rectangles $R \subset[0, T]^{2}$,

$$
\sum_{i=1}^{n} \omega\left(R_{i}\right) \leq \omega(R) \quad \text { whenever }\left\{R_{i}, 1 \leq i \leq n\right\} \in \Pi(R) .
$$

Now let $f \in \mathcal{C}^{p-\operatorname{var}}\left([0, T]^{2} ; E\right), \varepsilon>0$ and $R \subset[0, T]^{2}$. Then $\omega_{f, \rho+\varepsilon}(R)$ defined as

$$
\omega_{f, \rho+\varepsilon}(R):=\sup _{\Pi(R)} \sum_{i}\left|f\left(\begin{array}{ll}
a_{i} & b_{i} \\
c_{i} & d_{i}
\end{array}\right)\right|^{\rho+\varepsilon},
$$

is a $2 \mathrm{D}$ control [19].

REMARK 2.9. Note that $\Pi(R)$ includes partitions which are not grid-like, in contrast to (19). Furthermore, we use $\omega_{f, \rho+\varepsilon}([s, t] \times[u, v])$ instead of what seems to be the more natural choice $\|f\|_{\rho \text {-var; }[s, t] \times[u, v]}^{\rho}$ because the latter is actually not super-additive, and is thus not a control; see [19].

However, there exists a finite constant depending on $\varepsilon$ such that

$$
\omega_{f, \rho+\varepsilon}([s, t] \times[u, v]) \leq C_{\varepsilon}\|f\|_{\rho-\operatorname{var} ;[s, t] \times[u, v]}^{\rho+\varepsilon}<\infty,
$$

for all $[s, t] \times[u, v] \subset[0, T]^{2}$. The reason to use $\rho+\varepsilon$ regularity instead of $\rho$ is that otherwise (22) might be infinite; cf. Example 1 in [19]. 
DEFINITION 2.10. We say that the 2D Young-Stieltjes integral of $f$ with respect to $g$ exists if there exists a scalar $I(f, g) \in \mathbb{R}$ such that

$$
\lim _{\|\pi\| \rightarrow 0}\left|\sum_{i, j} f\left(u_{i}, v_{j}\right) g\left(\begin{array}{ll}
u_{i} & u_{i+1} \\
v_{j} & v_{j+1}
\end{array}\right)-I(f, g)\right| \rightarrow 0,
$$

that is, for each $\varepsilon>0$, there exists a $\delta>0$ such that for all partitions $\pi=\left\{\left(u_{i}, v_{j}\right)\right\}$ of $[0, T]^{2}$ with $\|\pi\|<\delta$, the quantity on the left of (23) is less than $\varepsilon$. In this case, we use $\int_{[0, T]^{2}} f \mathrm{~d} g$ to denote $I(f, g)$, or $\int_{[s, t] \times[u, v]} f \mathrm{~d} g$ whenever we restrict ourselves to any particular subset $[s, t] \times[u, v]$ of $[0, T]^{2}$.

DEFINITION 2.11. We say that $f \in V^{p \text {-var }}([s, t] \times[u, v])$ and $g \in$ $V^{q-\operatorname{var}}([s, t] \times[u, v])$ have complementary regularity if $\frac{1}{p}+\frac{1}{q}>1$.

The significance of this definition lies in the following theorem, which gives the existence of the Young-Stieltjes integral and Young's inequality in two dimensions; see $[14,18,27]$ for the one-dimensional version.

THEOREM 2.12. Let $f \in V^{p-\operatorname{var}}([s, t] \times[u, v])$ and $g \in V^{q-\operatorname{var}}([s, t] \times[u, v])$ have complementary regularity. We also assume that $f(s, \cdot)$ and $f(\cdot, u)$ have $f$ nite $p$-variation, and that $f$ and $g$ have no common discontinuities. Then the $2 D$ Young-Stieltjes integral exists and the following Young's inequality holds:

$$
\left|\int_{[s, t] \times[u, v]} f \mathrm{~d} g\right| \leq C_{p, q}\|\mid f\|\|g\|_{q-\operatorname{var},[s, t] \times[u, v]},
$$

where

$$
\|\| f\|=|f(s, u)|+\| f(s, \cdot)\left\|_{p \text {-var; }[u, v]}+\right\| f(\cdot, u)\left\|_{p \text {-var; }[s, t]}+\right\| f \|_{p \text {-var, }[s, t] \times[u, v]} .
$$

ProOF. See $[17,36]$.

2.2. Gaussian rough paths. We will work with a stochastic process

$$
X_{t}=\left(X_{t}^{(1)}, \ldots, X_{t}^{(d)}\right), \quad t \in[0, T],
$$

which denotes a centered (i.e., zero-mean), continuous Gaussian process in $\mathbb{R}^{d}$ with i.i.d. components and starting at the origin.

This process is defined on the canonical probability space $(\Omega, \mathcal{F}, \mathbb{P})$, where $\Omega=\mathcal{C}\left([0, T] ; \mathbb{R}^{d}\right)$, the space of continuous $\mathbb{R}^{d}$-valued paths equipped with the supremum topology, $\mathcal{F}$ is the Borel $\sigma$-algebra and $\mathbb{P}$ is the unique Borel measure under which $X(\omega)=\left(\omega_{t}\right)_{t \in[0, T]}$ has the specified Gaussian distribution. We will use

$$
R(s, t):=\mathbb{E}\left[X_{s}^{(1)} X_{t}^{(1)}\right]
$$


to denote the covariance function common to the components. The variance $R(t, t)$ will be denoted simply by $R(t)$, and we will also use the notation

$$
\sigma^{2}(s, t):=R\left(\begin{array}{ll}
s & t \\
s & t
\end{array}\right)=\mathbb{E}\left[\left(X_{s, t}^{(1)}\right)^{2}\right]
$$

recall the definition of the rectangular increment in (20).

The triple $\left(\Omega, \mathcal{H}^{d}, \mathbb{P}\right)$ denotes the abstract Wiener space associated to $X$, where $\mathcal{H}^{d}=\bigoplus_{i=1}^{d} \mathcal{H}$ is the Cameron-Martin space (or reproducing kernel Hilbert space). The Cameron-Martin space, which is densely and continuously embedded in $\Omega$, is the completion of the linear span of the functions

$$
\left\{R(t, \cdot)^{(u)}:=R(t, \cdot) e_{u} \mid t \in[0, T], u=1, \ldots, d\right\}
$$

under the inner-product

$$
\left\langle R(t, \cdot)^{(u)}, R(s, \cdot)^{(v)}\right\rangle_{\mathcal{H}^{d}}=\delta_{u v} R(t, s), \quad u, v=1, \ldots, d .
$$

By definition, $\mathcal{H}^{d}$ satisfies the following reproducing property; for any $f=$ $\left(f^{(1)}, \ldots, f^{(d)}\right) \in \mathcal{H}^{d}$,

$$
\left\langle f ., R(t, \cdot)^{(u)}\right\rangle_{\mathcal{H}^{d}}=f_{t}^{(u)}, \quad t \in[0, T] .
$$

We assume that there exists $\rho<2$ such that $R$ has finite 2D $\rho$-variation. The following theorem in [17] (see also [11] in the case of fractional Brownian motion) then shows that one can canonically lift $X$ via its piecewise linear approximants $X^{\pi}$ to a geometric $p$-rough path for $p>2 \rho$.

THEOREM 2.13. Assume $X$ is a centered continuous $\mathbb{R}^{d}$-valued Gaussian process with i.i.d. components. Let $\rho \in[1,2)$ and assume that the covariance function has finite $2 D \rho$-variation:

(i) (Existence) There exists a random variable $\mathbf{X}=\left(1, \mathbf{X}^{1}, \mathbf{X}^{2}, \mathbf{X}^{3}\right)$ on $(\Omega, \mathcal{F}$, $\mathbb{P})$ which takes values almost surely in $\mathcal{C}^{0, p-\operatorname{var}}\left([0, T] ; G^{3}\left(\mathbb{R}^{d}\right)\right)$ for $p>2 \rho$, that $i s$, the set of geometric $p$-rough paths for $p \in(2 \rho, 4)$, and which lifts the Gaussian process $X$ in the sense that $\mathbf{X}_{s, t}^{1}=X_{t}-X_{s}$ almost surely for all $s, t \in[0, T]$.

(ii) (Uniqueness and consistency) The lift $\mathbf{X}$ is unique in the sense that it is the $d_{p-v a r}$-limit in $L^{q}(\Omega), q \in[1, \infty)$, of any sequence $S_{\lfloor p\rfloor}\left(X^{\pi}\right)$ with $\|\pi\| \rightarrow 0$. Furthermore, if $X$ has a.s. sample paths of finite $[1,2)$-variation, $\mathbf{X}$ coincides with the signature of $X$.

Moreover, Proposition 17 in [17] shows that for all $h \in \mathcal{H}^{d}$,

$$
\|h\|_{\rho \text {-var; }[0, T]} \leq\|h\|_{\mathcal{H}^{d}} \sqrt{\|R\|_{\rho-\operatorname{var} ;[0, T]^{2}}},
$$

which implies that $\mathcal{H}^{d} \hookrightarrow \mathcal{C}^{\rho \text {-var }}\left([0, T] ; \mathbb{R}^{d}\right)$ whenever $R$ has finite $2 \mathrm{D} \rho$ variation. Thus if $\rho \in\left[1, \frac{3}{2}\right)$, corresponding to $2 \leq p<3$, we have complementary regularity between $X$ and any path in the Cameron-Martin space, that is, $\frac{1}{p}+\frac{1}{\rho}>1$. 
We will need to impose further conditions on the covariance function. For all $s, t \in[0, T]$, we assume there exists $C<\infty$ such that

$$
\|R(t, \cdot)-R(s, \cdot)\|_{\rho-\operatorname{var} ;[0, T]} \leq C|t-s|^{\frac{1}{\rho}} .
$$

This bound will be later used to control the $L^{2}(\Omega)$ norm of the iterated integrals. An immediate consequence of the bound is illustrated in the following lemma.

LEMMA 2.14. Let $X$ be a continuous, centered Gaussian process in $\mathbb{R}$ and assume its covariance function satisfies

$$
\|R(t, \cdot)-R(s, \cdot)\|_{q \text {-var; }[0, T]} \leq C|t-s|^{\frac{1}{\rho}} \quad \forall s<t \in[0, T],
$$

for some $q, \rho \geq 1$. Then:

(i) $R(t):=R(t, t)$ is of bounded $\rho$-variation.

(ii) For $p>2 \rho, X$ has a $\frac{1}{p}$-Hölder continuous modification.

ProOF. Let $f_{s, t}(\cdot)$ denote $R(t, \cdot)-R(s, \cdot)$. Then for any partition $\left\{t_{i}\right\}$ of $[0, T]$, we have

$$
\begin{aligned}
\sum_{i} \mid & R\left(t_{i+1}\right)-\left.R\left(t_{i}\right)\right|^{\rho} \\
& \leq \sum_{i}\left|R\left(t_{i+1}, t_{i+1}\right)-R\left(t_{i}, t_{i+1}\right)+R\left(t_{i}, t_{i+1}\right)-R\left(t_{i}, t_{i}\right)\right|^{\rho} \\
& \leq 2^{\rho-1} \sum_{i}\left|f_{t_{i}, t_{i+1}}\left(t_{i+1}\right)-f_{t_{i}, t_{i+1}}(0)\right|^{\rho}+\left|f_{t_{i}, t_{i+1}}\left(t_{i}\right)-f_{t_{i}, t_{i+1}}(0)\right|^{\rho} \\
& \leq 2^{\rho} \sum_{i}\left\|f_{t_{i}, t_{i+1}}(\cdot)\right\|_{q-\mathrm{var} ;[0, T]}^{\rho} \\
& \leq C \sum_{i}\left|t_{i+1}-t_{i}\right| \leq C T
\end{aligned}
$$

For the second part, for all $n \in \mathbb{N}$ we have

$$
\begin{aligned}
\mathbb{E}\left[X_{s, t}^{2 n}\right] \leq C_{n} \mathbb{E}\left[X_{s, t}^{2}\right]^{n} & \leq C_{n}\left|f_{s, t}(t)-f_{s, t}(s)\right|^{n} \\
& \leq C_{n}\|R(t, \cdot)-R(s, \cdot)\|_{q \text {-var; }[0, T]}^{n} \\
& \leq C_{n}|t-s|^{\frac{n}{\rho}}, \quad s<t \in[0, T] .
\end{aligned}
$$

By Kolmogorov's continuity theorem, there exists a $\gamma$-Hölder continuous modification of $X$ for all $\gamma<\frac{1}{2 \rho}$. 
2.3. Malliavin calculus. We will primarily work with the following Hilbert space which is isomorphic to $\mathcal{H}^{d}$.

DEFINITION 2.15. Let $\mathcal{H}_{1}^{d}$ denote the completion of the linear span of

$$
\left\{\mathbb{1}_{[0, t)}^{(u)}(\cdot):=\mathbb{1}_{[0, t)}(\cdot) e_{u} \mid t \in[0, T], u=1, \ldots, d\right\}
$$

(cf. [1, 29]) with respect to the inner-product given by

$$
\left\langle\mathbb{1}_{[0, t)}^{(u)}(\cdot), \mathbb{1}_{[0, s)}^{(v)}(\cdot)\right\rangle_{\mathcal{H}_{1}^{d}}=\delta_{u v} R(t, s) .
$$

Furthermore, let $\Phi: \mathcal{H}_{1}^{d} \rightarrow \mathcal{H}^{d}$ denote the Hilbert space isomorphism obtained from extending the map $\mathbb{1}_{[0, t)}^{(u)}(\cdot) \mapsto R(t, \cdot)^{(u)}, t \in[0, T], u=1, \ldots, d$.

We record some basic properties about the Malliavin calculus. For simplicity, we assume here that $d=1$. First, we recall that the map $\mathbb{1}_{[0, t)} \mapsto X_{t}$ extends to a unique linear isometry $I$ from $\mathcal{H}_{1}$ to $L^{2}(\Omega)$. It follows that $I(h)$ is a meanzero Gaussian random variable with variance $\|h\|_{\mathcal{H}_{1}}^{2}$. The set $\mathcal{S}$ of smooth cylinder functionals is the subset of random variables taking the form

$$
F=f\left(I\left(h_{1}\right), \ldots, I\left(h_{n}\right)\right),
$$

where $h_{1}, \ldots, h_{n} \in \mathcal{H}_{1}$ and $f: \mathbb{R}^{n} \rightarrow \mathbb{R}$ is infinitely differentiable with bounded derivatives of all orders. The Malliavin derivative $\mathcal{D} F$ is the $\mathcal{H}_{1}$-valued random variable which is defined for smooth cylinder functionals as follows:

$$
\mathcal{D} F:=\sum_{i=1}^{n} \frac{\partial f}{\partial x_{i}}\left(I\left(h_{1}\right), \ldots, I\left(h_{n}\right)\right) h_{i} .
$$

It can be shown that $\mathcal{D}$ is a closable operator; see, for example, Proposition 1.2.1 in [29]. For $p \geq 1$, we let $\mathbb{D}^{1, p}$ denote the closure of $\mathcal{S}$ with respect to the norm

$$
\|F\|_{1, p}^{p}:=\|F\|_{L^{p}(\Omega)}^{p}+\|\mathcal{D} F\|_{L^{p}\left(\Omega ; \mathcal{H}_{1}\right)}^{p} .
$$

If $K$ is a separable Hilbert space, the higher-order derivatives $\mathcal{D}^{n}$ and the corresponding Sobolev spaces $\mathbb{D}^{n, p}(K)$ can be defined iteratively.

Moving to the case $p=2$, for any $F$ in $\mathbb{D}^{1,2}$, we let $\mathcal{D}_{h} F:=\langle\mathcal{D} F, h\rangle_{\mathcal{H}_{1}}$. The divergence operator $\delta^{X}$ is defined to be the adjoint of $\mathcal{D}$. The domain of this operator consists of all $h \in L^{2}\left(\Omega ; \mathcal{H}_{1}\right)$ such that

$$
\left|\mathbb{E}\left[\mathcal{D}_{h} F\right]\right| \leq C\|F\|_{L^{2}(\Omega)}
$$

for all $F \in \mathcal{S}$, whereupon $\delta^{X}(h)$ is characterized as the unique random variable in $L^{2}(\Omega)$ for which

$$
\mathbb{E}\left[\langle\mathcal{D} F, h\rangle_{\mathcal{H}_{1}}\right]=\mathbb{E}\left[F \delta^{X}(h)\right]
$$


We will use the notation $\delta^{X}(h)$ and $\int_{0}^{T} h_{s} \mathrm{~d} X_{S}$ interchangeably. It is well known that the domain of $\delta^{X}$ contains $\mathbb{D}^{1,2}\left(\mathcal{H}_{1}\right)$; see, for example, Proposition 1.3.1 in [29].

Fixing a multi-index $a=\left(a_{1}, \ldots, a_{M}\right)$ where $|a|:=\sum_{i=1}^{M} a_{i}=n$, we define $I_{n}: \mathcal{H}_{1}^{\otimes n} \rightarrow \mathbb{R}$ as follows:

$$
I_{n}\left(h_{1}^{\otimes a_{1}} \otimes \cdots \otimes h_{M}^{\otimes a_{M}}\right)=a ! \prod_{i=1}^{M} H_{a_{i}}\left(\delta^{X}\left(h_{i}\right)\right),
$$

where $a !:=\prod_{i=1}^{M} a_{i} !$ and $H_{m}(x)$ denotes the $m$ th Hermite polynomial. The following duality formula is then classical

$$
\mathbb{E}\left[F I_{n}(h)\right]=\mathbb{E}\left[\left\langle D^{n} F, h\right\rangle_{\mathcal{H}_{1}^{\otimes n}}\right] .
$$

For $f \in \mathcal{H}_{1}^{\otimes n}, g \in \mathcal{H}_{1}^{\otimes m}$, both $f$ and $g$ symmetric, we also have the following product formula (cf. Proposition 1.1.3 in [29]):

$$
I_{n}(f) I_{m}(g)=\sum_{r=0}^{n \wedge m} r !\left(\begin{array}{l}
n \\
r
\end{array}\right)\left(\begin{array}{l}
m \\
r
\end{array}\right) I_{n+m-2 r}\left(f \tilde{\otimes}_{r} g\right) .
$$

Here, $f \tilde{\otimes}_{r} g$ denotes the symmetrization of the tensor $f \otimes_{r} g$, which in turn denotes the contraction of $f$ and $g$ of order $r$; that is, given an orthonormal basis $\left\{h_{m}\right\}$ of $\mathcal{H}_{1}$,

$$
f \otimes_{r} g:=\sum_{k_{1}, \ldots, k_{r}=1}^{\infty}\left\langle f, h_{k_{1}} \otimes \cdots \otimes h_{k_{r}}\right\rangle_{\mathcal{H}_{1}^{\otimes r}} \otimes\left\langle g, h_{k_{1}} \otimes \cdots \otimes h_{k_{r}}\right\rangle_{\mathcal{H}_{1}^{\otimes r}} \in \mathcal{H}_{1}^{\otimes(n+m-2 r)}
$$

cf. [28].

REMARK 2.16. One can also define operators equivalent to $\mathcal{D}$ and $\delta^{X}$ directly on the abstract Wiener space $(\Omega, \mathcal{H}, \mathbb{P})$. To make the presentation clear we summarize the correspondence here. First, for every $l$ in the topological dual $\Omega^{*}=\mathcal{C}([0, T], \mathbb{R})^{*}$, there exists a unique $h_{l}$ in $\mathcal{H}$ such that $l(h)=\left\langle h_{l}, h\right\rangle$. Under this identification, the random variable $\mathcal{I}\left(h_{l}\right): \omega \mapsto l(\omega)$ is a centered normal random variable with variance $\left\|h_{l}\right\|_{\mathcal{H}}^{2}$. Second, it can be shown that the set $\left\{h_{l}: l \in \Omega^{*}\right\}$ is dense in $\mathcal{H}$, whereupon $\mathcal{I}$ extends uniquely to an isometry between $\mathcal{H}$ and $L^{2}(\Omega)$, and is called the Paley-Wiener map. It is simple to see that $I$ and $\mathcal{I}$ are related by $I(h)=\mathcal{I}(\Phi(h))$ for all $h \in \mathcal{H}_{1}$ and, therefore, any smooth cylinder functional $F$ can be represented as $F=f\left(\mathcal{I}\left(\Phi\left(h_{1}\right)\right), \ldots, \mathcal{I}\left(\Phi\left(h_{n}\right)\right)\right)$, and a derivative operator $\mathbf{D}$ can be defined analogously to $\mathcal{D}$ by setting

$$
\mathbf{D} F:=\sum_{i=1}^{n} \frac{\partial f}{\partial x_{i}}\left(\mathcal{I}\left(\Phi\left(h_{1}\right)\right), \ldots, \mathcal{I}\left(\Phi\left(h_{n}\right)\right)\right) \Phi\left(h_{i}\right)=\Phi(\mathcal{D} F) .
$$

This implies that

$$
\mathbf{D}_{\Phi(h)} F=\langle\mathbf{D} F, \Phi(h)\rangle_{\mathcal{H}}=\langle\mathcal{D} F, h\rangle_{\mathcal{H}_{1}}=\mathcal{D}_{h} F \quad \forall h \in \mathcal{H}_{1} .
$$


The exposition above presents Shigekawa's definition of the Sobolev-type space $\mathbb{D}^{n, p}(K)$ for $K$-valued Wiener functionals, where $K$ is a separable Hilbert space. Although this is the one most often used in the literature, there are equivalent characterizations of these spaces. One of these, which is attributed to Kusuoka and Stroock (cf. [35]), is especially convenient to study stochastic differential equations for which bounds on the directional derivatives can be computed explicitly. The definition relies on two properties. First, a measurable function $F: \Omega \rightarrow K$ is called ray absolutely continuous (RAC) if for every $k \in \mathcal{H}$, there exists a measurable map $\tilde{F}_{k}: \Omega \rightarrow K$ such that

$$
F(\cdot)=\tilde{F}_{k}(\cdot), \quad \mathbb{P} \text {-a.s. }
$$

and for any $\omega \in \Omega$ the function $s \mapsto \tilde{F}_{k}(\omega+s k)$ is locally ${ }^{3}$ absolutely continuous in $s \in \mathbb{R}$. Second, $F$ has the property of being stochastically Gâteaux differentiable (SGD) if there exists a measurable $G: \Omega \rightarrow \mathcal{L}_{\mathrm{HS}}(\mathcal{H}, K)$, such that for any $k \in \mathcal{H}$

$$
\frac{1}{\varepsilon}[F(\cdot+\varepsilon k)-F(\cdot)] \stackrel{\mathbb{P}}{\rightarrow} G(\omega)(k) \quad \text { as } t \rightarrow 0,
$$

where $\mathcal{L}_{\mathrm{HS}}(\mathcal{H}, K)$ denotes the space of linear Hilbert-Schmidt operators from $\mathcal{H}$ to $K$. In this case, the derivative $G$ is unique $\mathbb{P}$-a.s. and we denote it by $\mathcal{D}_{\mathrm{KS}} F$. Higher-order derivatives are defined inductively in the obvious way: if $\mathcal{D}_{\mathrm{KS}}^{n-1} F$ is SGD then $\mathcal{D}_{\mathrm{KS}}^{n} F:=\mathcal{D}_{\mathrm{KS}}\left(\mathcal{D}_{\mathrm{KS}}^{n-1} F\right)$.

Next, we define the spaces $\mathbb{D}_{\mathrm{KS}}^{n, p}(K)$ for $1<p<\infty$ inductively, first for $n=1$ by setting

$$
\mathbb{D}_{\mathrm{KS}}^{1, p}(K):=\left\{F \in L^{p}(K): F \text { is } \mathrm{RAC} \text { and } \mathrm{SGD}, \mathcal{D}_{\mathrm{KS}} F \in L^{p}\left(\mathcal{L}_{\mathrm{HS}}(\mathcal{H}, K)\right)\right\},
$$

and then analogously for $n=2,3, \ldots$ by

$$
\mathbb{D}_{\mathrm{KS}}^{n, p}(K):=\left\{F \in \mathbb{D}_{\mathrm{KS}}^{n-1, p}(K): \mathcal{D}_{\mathrm{KS}} F \in \mathbb{D}_{\mathrm{KS}}^{n-1, p}\left(\mathcal{L}_{\mathrm{HS}}(\mathcal{H}, K)\right)\right\} .
$$

We have the following theorem.

THEOREM 2.17 (Theorem 3.1 in [35]). For $1<p<\infty$ and $n \in \mathbb{N}$, we have $\mathbb{D}_{\mathrm{KS}}^{n, p}(K)=\mathbb{D}^{n, p}(K)$, and for any element $F$ in this space, $\mathcal{D}_{\mathrm{KS}} F=\mathcal{D} F$ holds $\mathbb{P}$-a.s.

REMARK 2.18. By applying the same result iteratively it follows that $\mathcal{D}_{\mathrm{KS}}^{k} F=\mathcal{D}^{k} F$ holds $\mathbb{P}$-a.s. for $k=2, \ldots, n$.

\footnotetext{
${ }^{3}$ Local absolute continuity is important here and is a point often missed in the literature where RAC is sometimes stated by demanding that $s \mapsto \tilde{F}_{k}(\omega+s k)$ is absolutely continuous in $s \in \mathbb{R}$. See however Definition 8.2.3 and Theorem 8.5.1 in [3] for a proof that local absolute continuity is enough.
} 
2.4. Rough integration and controlled rough paths. In this subsection, we will review rough integration via the theory of controlled rough paths. We will develop the concepts in $p$-variation topology rather than the usual Hölder topology (cf. [20] and [14]), and henceforth, $\mathcal{U}, \mathcal{V}$ will denote finite-dimensional vector spaces.

We begin with the following definition.

Definition 2.19. Let $\mathbf{x}=\left(1, x, \mathbf{x}^{2}\right) \in \mathcal{C}^{p \text {-var }}\left([0, T] ; G^{2}\left(\mathbb{R}^{d}\right)\right)$. A pair of paths $\left(\phi, \phi^{\prime}\right)$, where $\phi \in \mathcal{C}^{p-\operatorname{var}}([0, T] ; \mathcal{U})$ and $\phi^{\prime} \in \mathcal{C}^{p \text {-var }}\left([0, T] ; \mathcal{L}\left(\mathbb{R}^{d} ; \mathcal{U}\right)\right)$, is said to be controlled by $\mathbf{x}$ if for all $s, t \in[0, T]$,

$$
\phi_{s, t}=\phi_{s}^{\prime} x_{s, t}+R_{s, t}^{\phi},
$$

where the remainder term satisfies

$$
R^{\phi} \in \mathcal{C}^{\frac{p}{2}-\operatorname{var}}([0, T] ; \mathcal{U}) .
$$

If we define the controlled variation norm as

$$
\|\phi\|_{p \text {-cvar }}:=\|\phi\|_{\mathcal{V}^{p} ;[0, T]}+\left\|\phi^{\prime}\right\|_{\mathcal{V}^{p} ;[0, T]}+\left\|R^{\phi}\right\|_{\frac{p}{2} \text {-var; }[0, T]},
$$

then the preceding definition says that $\left(\phi, \phi^{\prime}\right)$ is controlled by $\mathbf{x}$ if $\|\phi\|_{p \text {-cvar }}<\infty$.

THEOREM 2.20. Let $\mathbf{x}=\left(1, x, \mathbf{x}^{2}\right) \in \mathcal{C}^{p-v a r}\left([0, T] ; G^{2}\left(\mathbb{R}^{d}\right)\right)$, where $2 \leq$ $p<3$.

Let $\phi \in \mathcal{C}^{p-\operatorname{var}}\left([0, T] ; \mathcal{L}\left(\mathbb{R}^{d} ; \mathbb{R}^{e}\right)\right)$ and $\phi^{\prime} \in \mathcal{C}^{p-\operatorname{var}}\left([0, T] ; \mathcal{L}\left(\mathbb{R}^{d} ; \mathcal{L}\left(\mathbb{R}^{d} ; \mathbb{R}^{e}\right)\right)\right)$. If $\left(\phi, \phi^{\prime}\right)$ is controlled by $\mathbf{x}$, we can define the rough integral

$$
\int_{0}^{t} \phi_{r} \circ \mathrm{d} \mathbf{x}_{r}:=\lim _{\|\pi\| \rightarrow 0, \pi=\left\{0=r_{0}<\cdots<r_{n}=t\right\}} \sum_{i=0}^{n-1}\left(\phi_{r_{i}} x_{r_{i}, r_{i+1}}+\phi_{r_{i}}^{\prime} \mathbf{x}_{r_{i}, r_{i+1}}^{2}\right),
$$

where we have made use of the canonical identification $\mathcal{L}\left(\mathbb{R}^{d} ; \mathcal{L}\left(\mathbb{R}^{d} ; \mathbb{R}^{e}\right)\right) \simeq$ $\mathcal{L}\left(\mathbb{R}^{d} \otimes \mathbb{R}^{d} ; \mathbb{R}^{e}\right)$. Furthermore, denoting

$$
z_{t}:=\int_{0}^{t} \phi_{r} \circ \mathrm{d} \mathbf{x}_{r}, \quad z_{t}^{\prime}:=\phi_{t},
$$

$\left(z, z^{\prime}\right)$ is again controlled by $\mathbf{x}$, and we have the bound

$$
\|z\|_{p \text {-cvar }} \leq C_{p}\|\phi\|_{p \text {-cvar }}\left(1+\|x\|_{p \text {-var; }[0, T]}+\left\|\mathbf{x}^{2}\right\|_{\frac{p}{2} \text {-var; }[0, T]}\right) .
$$

The following propositions will provide us with various ways to construct controlled rough paths from existing ones.

Proposition 2.21. For $p \geq 2$, let

$$
\begin{aligned}
y & \in \mathcal{C}^{p-\operatorname{var}}([0, T] ; \mathcal{U}), \\
y^{\prime} & \in \mathcal{C}^{p-\operatorname{var}}\left([0, T] ; \mathcal{L}\left(\mathbb{R}^{d} ; \mathcal{U}\right)\right),
\end{aligned}
$$

and let $\phi$ be a $\mathcal{C}_{b}^{2}$ map from $\mathcal{U}$ to $\mathcal{V}$. 
Then $\phi(y) \in \mathcal{C}^{p \text {-var }}([0, T] ; \mathcal{V})$ and $\nabla \phi(y) y^{\prime} \in \mathcal{C}^{p-\operatorname{var}}\left([0, T] ; \mathcal{L}\left(\mathbb{R}^{d} ; \mathcal{V}\right)\right)$. Furthermore, if $\left(y, y^{\prime}\right)$ is controlled by $\mathbf{x} \in \mathcal{C}^{p-\operatorname{var}}\left([0, T] ; G^{2}\left(\mathbb{R}^{d}\right)\right)$, then $(\phi(y)$, $\left.\nabla \phi(y) y^{\prime}\right)$ is also controlled by $\mathbf{x}$ and we have

$$
\begin{aligned}
& \|\phi(y)\|_{p \text {-var; }[0, T]},\left\|\nabla \phi(y) y^{\prime}\right\|_{p \text {-var; }[0, T]} \\
& \quad \leq\|\phi\|_{\mathcal{C}_{b}^{2}}\|y\|_{\mathcal{V}^{p} ;[0, T]}\left(1+\left\|y^{\prime}\right\|_{\mathcal{V}^{p} ;[0, T]}\right),
\end{aligned}
$$

and

$$
\left\|R^{\phi(y)}\right\|_{\frac{p}{2}-\operatorname{var} ;[0, T]} \leq\|\phi\|_{\mathcal{C}_{b}^{2}}\left(\|y\|_{p-\operatorname{var} ;[0, T]}^{2}+\left\|R^{y}\right\|_{\frac{p}{2}-\operatorname{var} ;[0, T]}\right) .
$$

PROPOSITION 2.22 (Leibniz rule). For $p \geq 2$, let

$$
\begin{aligned}
\phi & \in \mathcal{C}^{p-\operatorname{var}}([0, T] ; \mathcal{L}(\mathcal{U} ; \mathcal{V})), \\
\phi^{\prime} & \in \mathcal{C}^{p-\operatorname{var}}\left([0, T] ; \mathcal{L}\left(\mathbb{R}^{d} ; \mathcal{L}(\mathcal{U} ; \mathcal{V})\right)\right),
\end{aligned}
$$

and we assume that $\left(\phi, \phi^{\prime}\right)$ is controlled by $\mathbf{x} \in \mathcal{C}^{p-\operatorname{var}}\left([0, T] ; G^{2}\left(\mathbb{R}^{d}\right)\right)$.

(i) Let $\psi \in \mathcal{C}^{p-\operatorname{var}}([0, T] ; \mathcal{U}), \psi^{\prime} \in \mathcal{C}^{p \text {-var }}\left([0, T] ; \mathcal{L}\left(\mathbb{R}^{d} ; \mathcal{U}\right)\right)$, and suppose that $\left(\psi, \psi^{\prime}\right)$ is controlled by $\mathbf{x}$. Then the path $\phi \psi \in \mathcal{C}^{p-v a r}([0, T] ; \mathcal{V})$ given by the composition of $\phi$ and $\psi$ is also controlled by $\mathbf{x}$, with derivative process $(\phi \psi)^{\prime}=$ $\phi^{\prime} \psi+\phi \psi^{\prime}$. In addition, we have the bound

$$
\|\phi \psi\|_{p \text {-cvar }} \leq 2\|\phi\|_{p \text {-cvar }}\|\psi\|_{p \text {-cvar }}
$$

(ii) Suppose that $\psi \in \mathcal{C}^{\frac{p}{2}-\operatorname{var}}([0, T] ; \mathcal{U})$. Then $\phi \psi \in \mathcal{C}^{p-\operatorname{var}}([0, T] ; \mathcal{V})$ is also controlled by $\mathbf{x}$, with derivative process $(\phi \psi)^{\prime}=\phi^{\prime} \psi$. Moreover, we have the bound

$$
\|\phi \psi\|_{p \text {-cvar }} \leq\|\phi\|_{p \text {-cvar }}\|\psi\|_{\mathcal{V}^{\frac{p}{2} ;[0, T]}} .
$$

REMARK 2.23. The second part of the proposition clearly holds true if the roles of $\phi$ and $\psi$ are reversed. Furthermore, it asserts that one can trade increased regularity in place of a controlled rough path structure in $\psi$ (or $\phi$ ) for the composition to remain a controlled rough path.

The proofs of the preceding theorem and propositions are routine and hence deferred to the Appendix.

2.5. Rough differential equations. Now consider the following equation:

$$
\mathrm{d} \mathbf{y}(t)=V(t, y(t)) \mathrm{d} \mathbf{x}(t), \quad y(0)=y_{0},
$$

where $V \in \mathcal{C}_{b}^{\lfloor p\rfloor}\left(\mathbb{R} \times \mathbb{R}^{e} ; \mathcal{L}\left(\mathbb{R}^{d} ; \mathbb{R}^{e}\right)\right)$ is a differentiable function with bounded derivatives up to degree $\lfloor p\rfloor$. Given $\mathbf{x} \in \mathcal{C}^{\infty}\left([0, T] ; G^{\lfloor p\rfloor}\left(\mathbb{R}^{d}\right)\right)$, the unique solution $\mathbf{y}=S_{\lfloor p\rfloor}(y)$ can be obtained simply by solving (38) as a regular ODE. Furthermore, we have the following theorem (see [25]). 
THEOREM 2.24 (Universal limit theorem). The Itô map $\mathcal{I}: \mathbf{x} \mapsto \mathbf{y}$ is continuous from $\mathcal{C}^{\infty}\left([0, T], G^{\lfloor p\rfloor}\left(\mathbb{R}^{d}\right)\right)$ to itself with respect to the $p$-variation topology and thus admits a unique extension to the space of all p-geometric rough paths $\mathcal{C}^{0, p-\operatorname{var}}\left([0, T], G^{\lfloor p\rfloor}\left(\mathbb{R}^{d}\right)\right)$.

The universal limit theorem allows one to transfer geometric results in the smooth case to geometric rough paths, that is, rough paths that satisfy the changeof-variable rule. This effectively allows a generalization of the Stratonovich integral to processes with higher $p$-variation.

We will mainly be considering RDEs with time-homogeneous vector fields driven by Gaussian geometric rough paths. Furthermore, although the RDE

$$
\mathrm{d} Y_{t}=V\left(Y_{t}\right) \circ \mathrm{d} \mathbf{X}_{t}, \quad Y_{0}=y_{0},
$$

outputs a full rough path $\mathbf{Y}_{t}$, we will be concerned only with the first level/pathlevel solution, which satisfies

$$
Y_{t}=y_{0}+\int_{0}^{t} V\left(Y_{s}\right) \circ \mathrm{d} \mathbf{X}_{s},
$$

and ignore the higher iterated integral terms. We will use the following notation; writing $V(Y)$ as the co-tensor

$$
\sum_{i=1}^{e} \sum_{j=1}^{d} V_{j}^{(i)}(Y) \mathrm{d} e_{i} \otimes \mathrm{d} e_{j} \in \mathbb{R}^{e} \otimes \mathbb{R}^{d},
$$

we will denote

$$
V^{2}(Y):=\sum_{i, m=1}^{e} \sum_{j, k=1}^{d} \frac{\partial V_{k}^{(i)}}{\partial e_{m}}(Y) V_{j}^{(m)}(Y) \mathrm{d} e_{i} \otimes \mathrm{d} e_{j} \otimes \mathrm{d} e_{k} \in \mathbb{R}^{e} \otimes \mathbb{R}^{d} \otimes \mathbb{R}^{d} .
$$

THEOREM 2.25. For all $s<t \in[0, T],\|Y\|_{p \text {-var; }[s, t]}$ is in $L^{q}(\Omega)$ for all $q>0$.

ProOF. From equation (10.15) in [18], we have

$$
\|Y\|_{p \text {-var; }[s, t]} \leq C_{p}\left(\|V\|_{C_{b}^{\lfloor p\rfloor}}\|\mathbf{X}\|_{p \text {-var; }[s, t]} \vee\|V\|_{C_{b}^{\lfloor p\rfloor}}^{p}\|\mathbf{X}\|_{p \text {-var; }[s, t]}^{p}\right),
$$

and $\|\mathbf{X}\|_{p \text {-var; [s,t] }}$ has moments of all orders; see Corollary 66 in [17].

We will now show that

$$
Y_{t} \in \mathbb{D}^{\infty}\left(\mathbb{R}^{e}\right):=\bigcap_{p>1} \bigcap_{k=1}^{\infty} \mathbb{D}^{k, p}\left(\mathbb{R}^{e}\right) \quad \text { for } t \geq 0,
$$


where $Y$ solves RDE (1) with smooth vector fields. To do so, we make use of the fact (cf. [7]) that there exists a measurable subset $\tilde{\Omega} \subset \Omega$ with $\mathbb{P}(\tilde{\Omega})=1$ such that for all $\omega \in \tilde{\Omega}$ we have the identity

$$
\mathbf{X}(\omega+\Phi(h))=T_{\Phi(h)} \mathbf{X}(\omega) \quad \forall h \in \mathcal{H}_{1}^{d},
$$

where $T_{\Phi(h)} \mathbf{X}$ denotes the rough path translation of $\mathbf{X}$ by $\Phi(h)$ (see [6]), which is well defined via Young-Stieltjes integration due to complementary regularity.

We then obtain

$$
Y_{t}(\omega+s \Phi(h)):=Y_{t}^{\mathbf{X}(\omega+s \Phi(h))}=Y_{t}^{T_{s} \Phi(h)} \mathbf{X}(\omega),
$$

which is smooth in $s$ (see Theorem 11.6 in [18]), and hence locally absolutely continuous. It follows that $Y_{t}$ is RAC; indeed, in this case we can even take the version $\tilde{F}_{\Phi(h)}$ in (30) to be independent of $\Phi(h)$. Using Theorem 2.17 , it is immediate from the definition of $\mathcal{D}_{\mathrm{KS}} Y_{t}$ and the directional derivatives that

$$
\mathcal{D}_{\mathrm{KS}} Y_{t}(\omega)(\Phi(h))=\mathcal{D}_{h} Y_{t}(\omega), \quad \mathbb{P} \text {-a.s. }
$$

for all $h \in \mathcal{H}_{1}^{d}$, and henceforth we will use the latter notation exclusively.

Moving on to the higher-order derivatives, given $h_{1}, \ldots, h_{n} \in \mathcal{H}_{1}^{d}$, we can take the directional derivatives of $Y_{t}$ in the directions $\Phi\left(h_{1}\right), \ldots, \Phi\left(h_{n}\right)$ in $\mathcal{H}^{d}$ by setting

$$
\mathcal{D}_{h_{1}, \ldots, h_{n}}^{n} Y_{t}:=\left.\frac{\partial^{n}}{\partial \varepsilon_{1} \cdots \partial \varepsilon_{n}} Y_{t}^{\varepsilon_{1}, \ldots, \varepsilon_{n}}\right|_{\varepsilon_{1}=\cdots=\varepsilon_{n}=0},
$$

where $Y_{t}^{\varepsilon_{1}, \ldots, \varepsilon_{n}}$ solves

$$
\mathrm{d} Y_{t}^{\varepsilon_{1}, \ldots, \varepsilon_{n}}=V\left(Y_{t}^{\varepsilon_{1}, \ldots, \varepsilon_{n}}\right) \circ \mathrm{d}\left(T_{\varepsilon_{1}} \Phi\left(h_{1}\right)+\cdots+\varepsilon_{n} \Phi\left(h_{n}\right) \mathbf{X}\right)_{t}, \quad Y_{0}^{\varepsilon_{1}, \ldots, \varepsilon_{n}}=y_{0} .
$$

The path (40) again has finite $p$-variation and in Section 3.1, we will give it an explicit expression in terms of a sum of rough integrals and/or Young-Stieltjes integrals when $n \geq 2$. It only remains to show that these derivatives are HilbertSchmidt operators with norms having moments of all orders, and this has been proved in [23].

When $n=1$ the first-order derivative is given by (cf. $[6,18]$ )

$$
\mathcal{D}_{h} Y_{t}=\int_{0}^{t} J_{t}^{\mathbf{X}}\left(J_{s}^{\mathbf{X}}\right)^{-1} V\left(Y_{s}\right) \mathrm{d} \Phi(h)(s) .
$$

Here, $J_{t}^{\mathbf{X}}$ denotes the Jacobian of the flow map $y_{0} \rightarrow Y_{t}$ and satisfies

$$
\mathrm{d} J_{t}^{\mathbf{X}}=\nabla V\left(Y_{t}\right)\left(\operatorname{od} \mathbf{X}_{t}\right) J_{t}^{\mathbf{X}}, \quad J_{0}^{\mathbf{X}}=\mathcal{I}_{e} .
$$

On occasion, we will use the shorthand

$$
J_{t \leftarrow s}^{\mathbf{X}}:=J_{t}^{\mathbf{X}}\left(J_{s}^{\mathbf{X}}\right)^{-1}, \quad 0 \leq s<t \leq T,
$$


and for future reference, we also note that its inverse $\left(J^{\mathbf{X}}\right)^{-1}$ satisfies

$$
\mathrm{d}\left(J_{t}^{\mathbf{X}}\right)^{-1}=-\left(J_{t}^{\mathbf{X}}\right)^{-1} \nabla V\left(Y_{t}\right)\left(\operatorname{od} \mathbf{X}_{t}\right), \quad\left(J_{0}^{\mathbf{X}}\right)^{-1}=\mathcal{I}_{e} .
$$

To bound the Jacobian, we will need the following definitions. Following [10] we define, for a given interval $[s, t] \subset[0, T]$ and $\beta>0$, the so-called greedy sequence $\left\{\tau_{i}(\beta)\right\}$, a finite increasing sequence given by

$$
\begin{aligned}
\tau_{0}(\beta) & =s, \\
\tau_{i+1}(\beta) & =\inf \left\{u \in\left(\tau_{i}, t\right] \mid\|\mathbf{X}\|_{p \text {-var; }\left[\tau_{i}, u\right]}^{p} \geq \beta\right\} \wedge t .
\end{aligned}
$$

We then denote

$$
N_{\beta ;[s, t]}^{\mathbf{X}}:=\sup \left\{n \in \mathbb{N} \cup\{0\} \mid \tau_{n}(\beta)<t\right\},
$$

and note the following theorem.

THEOREM 2.26. Let $X$ be an $\mathbb{R}^{d}$-valued centered Gaussian process with i.i.d. components. For $1 \leq p<4$, assume that $X$ has a natural lift to $\mathbf{X} \in$ $\mathcal{C}^{0, p-\operatorname{var}}\left([0, T] ; G^{\lfloor p\rfloor}\left(\mathbb{R}^{d}\right)\right)$, and that $\mathcal{H}^{d} \hookrightarrow \mathcal{C}^{q-\operatorname{var}}\left([0, T] ; \mathbb{R}^{d}\right)$, where $\frac{1}{p}+\frac{1}{q}>1$. Then we have

$$
\mathbb{P}\left[N_{\beta ;[0, T]}^{\mathbf{X}}>n\right] \leq C_{1} \exp \left(-C_{2} \beta^{2} n^{\frac{2}{q}}\right)
$$

PROOF. See the proof of Theorem 6.3 in [10].

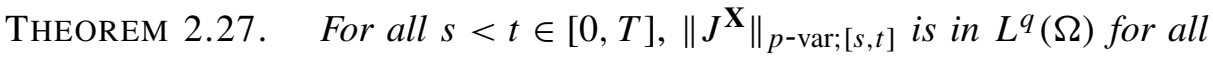
$q>0$.

Proof. Using the fact that $N_{1 ;[s, t]}^{\mathbf{X}}$ has Gaussian tails from the previous theorem, we see that $\mathbb{E}\left[\exp \left(C_{2} q N_{1 ;[s, t]}^{\mathbf{X}}\right)\right]<\infty$ for all $q>0, s<t \in[0, T]$. Now from equation (4.10) in [10], we have the bound

$$
\left\|J^{\mathbf{X}}\right\|_{p \text {-var; }[s, t]} \leq C_{1}\|\mathbf{X}\|_{p \text {-var; }[s, t]} \exp \left(C_{2} N_{1 ;[s, t]}^{\mathbf{X}}\right) .
$$

The statement of the theorem then follows immediately using Cauchy-Schwarz since $\|\mathbf{X}\|_{p \text {-var; }[s, t]}$ also has moments of all orders.

3. High-order directional derivatives for solutions to RDEs. We first begin with the following theorem.

THEOREM 3.1. Consider the system of RDEs

$$
\begin{aligned}
\mathrm{d} y_{t} & =V\left(y_{t}\right) \circ \mathrm{d} \mathbf{x}_{t}, \quad y_{0}=a \in \mathbb{R}^{e}, \\
\mathrm{~d} J_{t}^{\mathbf{x}} & =\nabla V\left(y_{t}\right)\left(\operatorname{od} \mathbf{x}_{t}\right) J_{t}^{\mathbf{x}}, \quad J_{0}^{\mathbf{x}}=\mathcal{I}_{e},
\end{aligned}
$$


where $\mathbf{x}=\left(1, x, \mathbf{x}^{2}\right) \in \mathcal{C}^{p-\operatorname{var}}\left([0, T] ; G^{2}\left(\mathbb{R}^{d}\right)\right), 2 \leq p<3$, and $V$ is in $\mathcal{C}_{b}^{3}\left(\mathbb{R}^{e} ; \mathbb{R}^{e} \otimes\right.$ $\left.\mathbb{R}^{d}\right)$.

In this case, both $(y, V(y))$ and $\left(J^{\mathbf{x}},\left(J^{\mathbf{x}}\right)^{\prime}\right)$ are controlled by $\mathbf{x}$. Moreover, we have the bounds

$$
\|y\|_{p-\mathrm{cvar}} \leq C_{p}\left(1+\|V\|_{\mathcal{C}_{b}^{2}}\right)^{4}\left(1+\|\mathbf{x}\|_{p \text {-var; }[0, T]}\right)^{3},
$$

and

$$
\left\|J^{\mathbf{x}}\right\|_{p-\mathrm{cvar}} \leq C_{1}\left(1+\exp \left(C_{2} N_{1 ;[0, T]}^{\mathbf{x}}\right)\right)^{4}\left(1+\|\mathbf{x}\|_{p \text {-var; }[0, T]}\right)^{3},
$$

where $C_{1}, C_{2}$ depend on $p$ and $\|V\|_{\mathcal{C}_{b}^{3}}$.

PROOF. (i) From Corollary 10.15 in [18], for $\gamma>p$ and $s, t \in[0, T]$, we have

$$
\left|y_{s, t}-V\left(y_{s}\right) x_{s, t}-V^{2}\left(y_{s}\right) \mathbf{x}_{s, t}^{2}\right| \leq C_{p}\left(\|V\|_{\mathcal{C}_{b}^{2}}\|\mathbf{x}\|_{p \text {-var; }[s, t]}\right)^{\gamma} .
$$

This implies that

$$
\begin{aligned}
\left|R_{s, t}^{y}\right|^{\frac{p}{2}} & \leq C_{p}\left(\left|V^{2}\left(y_{s}\right) \mathbf{x}_{s, t}^{2}\right|^{\frac{p}{2}}+\left(\|V\|_{\mathcal{C}_{b}^{2}}\|\mathbf{x}\|_{p \text {-var; }[s, t]}\right)^{\frac{\gamma p}{2}}\right) \\
& \leq C_{p}\left(\|V\|_{\mathcal{C}_{b}^{2}}^{p}\|\mathbf{x}\|_{p \text {-var; }[s, t]}^{p}+\|V\|_{\mathcal{C}_{b}^{2}}^{\frac{\gamma p}{2}}\|\mathbf{x}\|_{p-\operatorname{var} ;[s, t]}^{\frac{\gamma p}{2}}\right),
\end{aligned}
$$

and thus

$$
\left\|R^{y}\right\|_{\frac{p}{2}-\operatorname{var} ;[0, T]} \leq C_{p}\left(\|V\|_{\mathcal{C}_{b}^{2}}^{2}\|\mathbf{x}\|_{p-\operatorname{var} ;[0, T]}^{2} \vee\|V\|_{\mathcal{C}_{b}^{2}}^{\gamma}\|\mathbf{x}\|_{p \text {-var; }[0, T]}^{\gamma}\right),
$$

from the super-additivity of the right-hand side of (48). We will choose $\gamma$ to be in the interval $(p, 3)$, and since

$$
\|V(y)\|_{p-\operatorname{var} ;[0, T]} \leq\|V\|_{\mathcal{C}_{b}^{2}}\|y\|_{p-\operatorname{var} ;[0, T]}
$$

and

$$
\|y\|_{p \text {-var; }[0, T]} \leq C_{p}\left(\|V\|_{\mathcal{C}_{b}^{2}}\|\mathbf{x}\|_{p \text {-var; [0,T] }} \vee\|V\|_{\mathcal{C}_{b}^{2}}^{p}\|\mathbf{x}\|_{p \text {-var;[0,T] }}^{p}\right),
$$

we obtain (46).

From Proposition 5 in [15], we have

$$
\left\|J^{\mathbf{x}}\right\|_{p \text {-var;[0,T] }} \leq \exp \left(C_{\left.p,\|V\|_{\mathcal{C}_{b}^{3}}\left(N_{1 ;[0, T]}^{\mathbf{x}}+1\right)\right)}\right.
$$

which gives us

$$
\left\|J^{\mathbf{x}}\right\|_{\infty} \leq 1+\exp \left(C_{p,\|V\|_{\mathcal{C}_{b}^{3}}}\left(N_{1 ;[0, T]}^{\mathbf{x}}+1\right)\right)=: 1+M
$$

For each $i=1, \ldots, d$, we can construct $U_{i} \in \mathcal{C}_{b}^{3}\left(\mathbb{R}^{e} \times \mathbb{R}^{e^{2}} ; \mathbb{R}^{e} \otimes \mathbb{R}^{d}\right)$ which is equal to the vector field $(y, z) \mapsto \nabla V_{i}(y) z$ on the set $\mathcal{W}_{1}=\left\{z \in \mathbb{R}^{e^{2}}|| z \mid \leq M+1\right\}$ and 
vanishes outside the set $\mathcal{W}_{2}=\left\{z \in \mathbb{R}^{e^{2}}|| z \mid<M+2\right\}$. Hence, we have

$$
\begin{aligned}
\left\|U_{i}\right\|_{\mathcal{C}_{b}^{3}} & \leq \sup _{z \in \mathcal{W}_{2}}\left\|\nabla V_{i}(\cdot) z\right\|_{\infty}+\left\|\nabla V_{i}(\cdot)\right\|_{\infty} \\
& =\|V\|_{\mathcal{C}_{b}^{3}}(M+3), \quad i=1, \ldots, d .
\end{aligned}
$$

Then the solution to

$$
\begin{aligned}
\mathrm{d} y_{t} & =V\left(y_{t}\right) \circ \mathrm{d} \mathbf{x}_{t}, \quad y_{0}=a \\
\mathrm{~d} J_{t}^{\mathbf{x}} & =U\left(y_{t}, J_{t}^{\mathbf{x}}\right) \circ \mathrm{d} \mathbf{x}_{t}, \quad J_{0}^{\mathbf{x}}=\mathcal{I}_{e},
\end{aligned}
$$

where $U=\left(U_{1}, \ldots, U_{d}\right)$, will be the same as the solution to the original system on $\mathbb{R}^{e} \times \mathcal{W}_{1}$, and it can be rewritten as

$$
\mathrm{d} \tilde{y}_{t}=\tilde{V}\left(\tilde{y}_{t}\right) \circ \mathrm{d} \mathbf{x}_{t}, \quad \tilde{y}_{0}=\left(a, \mathcal{I}_{e}\right),
$$

where $\tilde{y}=\left(y, J^{\mathbf{x}}\right) \in \mathbb{R}^{e} \times \mathbb{R}^{e^{2}}$ and $\|\tilde{V}\|_{\mathcal{C}_{b}^{3}} \leq\|V\|_{\mathcal{C}_{b}^{3}}(M+3)$.

Hence, we can apply (46) to obtain

$$
\|\tilde{y}\|_{p \text {-cvar }} \leq C_{p}\left(1+\|V\|_{\mathcal{C}_{b}^{3}}(M+3)\right)^{4}\left(1+\|\mathbf{x}\|_{p \text {-var; }[0, T]}\right)^{3},
$$

and since $J^{\mathbf{x}}$ is a component of $\tilde{y}$, we obtain (47).

3.1. Upper bounds on the high-order directional derivatives. We now use the preceding theorem as well as results on controlled rough paths from Section 2.4 to obtain upper bounds on the directional derivative

$$
\mathcal{D}_{g_{1}, \ldots, g_{n}}^{n} y_{t}:=\left.\frac{\partial^{n}}{\partial \varepsilon_{1} \cdots \partial \varepsilon_{n}} y_{t}^{\varepsilon_{1}, \ldots \varepsilon_{n}}\right|_{\varepsilon_{1}=\cdots=\varepsilon_{n}=0},
$$

where the driving rough path $\mathbf{x}$ is perturbed in the directions $g_{1}, \ldots, g_{n}$ which are now assumed to be paths having complementary regularity with $\mathbf{x}$. To condense the notation, we will write $\mathcal{D}_{A}^{|A|} y_{t}$, for any subset $A$ of $\left\{g_{1}, \ldots, g_{n}\right\}$, noting that the symmetry of the derivative ensures this is well defined. For $i \in\{1, \ldots, n\}$, we then let $A_{i}^{n}(\cdot):[0, T] \rightarrow\left(\mathbb{R}^{e}\right)^{\otimes i}$ be defined by

$$
A_{i}^{n}(t):=\sum_{\pi=\left\{\pi_{1}, \ldots, \pi_{i}\right\} \in \mathcal{P}\left(\left\{g_{1}, \ldots, g_{n}\right\}\right)} \mathcal{D}_{\pi_{1}}^{\left|\pi_{1}\right|} y_{t} \tilde{\otimes} \cdots \tilde{\otimes} \mathcal{D}_{\pi_{i}}^{\left|\pi_{i}\right|} y_{t}, \quad t \in[0, T] .
$$

Here, $\tilde{\otimes}$ denotes the symmetric tensor product, and the summation is over the set of all partitions of $\left\{g_{1}, \ldots, g_{n}\right\}$ containing exactly $i$ elements. For all $i \in\{1, \ldots, n-$ $1\}$ and $j \in\{1, \ldots, n\}$, we also let $B_{i, j}^{n}(\cdot):[0, T] \rightarrow\left(\mathbb{R}^{e}\right)^{\otimes i}$ be defined by

$$
B_{i, j}^{n}(t):=\sum_{\pi=\left\{\pi_{1}, \ldots, \pi_{i}\right\} \in \mathcal{P}\left(\left\{g_{1}, \ldots, g_{j-1}, g_{j+1}, \ldots, g_{n}\right\}\right)} \mathcal{D}_{\pi_{1}}^{\left|\pi_{1}\right|} y_{t} \tilde{\otimes} \cdots \tilde{\otimes} \mathcal{D}_{\pi_{i}}^{\left|\pi_{i}\right|} y_{t} .
$$

The following result gives an integral equation for the formula for $\mathcal{D}_{g_{1}, \ldots, g_{n}}^{n} y_{t}$ in terms of these paths (cf. [8, 21, 23]). 
THEOREM 3.2. Let $p \geq 1$ and $q \geq 1$ be such that $1 / p+1 / q>1$, and let $n \in \mathbb{N}$ such that $n \geq 2$. Assume $\mathbf{x} \in \mathcal{C}^{0, \bar{p}-\mathrm{var}}\left([0, T] ; G^{\lfloor p\rfloor}\left(\mathbb{R}^{d}\right)\right)$ and suppose $y$ is the path-level solution to the RDE

$$
\mathrm{d} y_{t}=V\left(y_{t}\right) \circ \mathrm{d} \mathbf{x}_{t}, \quad y_{0} \in \mathbb{R}^{e} \text { given, }
$$

where $V \in \mathcal{C}_{b}^{\lfloor p\rfloor+n}\left(\mathbb{R}^{e} ; \mathbb{R}^{e} \otimes \mathbb{R}^{d}\right)$. Suppose that $g_{1}, \ldots, g_{n} \in C^{q-\operatorname{var}}\left([0, T] ; \mathbb{R}^{d}\right)$. Then the nth-order directional derivative (50) satisfies the $R D E$

$$
\begin{aligned}
\mathrm{d} \mathcal{D}_{g_{1}, \ldots, g_{n}}^{n} y_{t}= & \sum_{i=1}^{n} \nabla^{i} V\left(y_{t}\right) A_{i}^{n}(t) \circ \mathrm{d} \mathbf{x}_{t} \\
& +\sum_{i=1}^{n-1} \sum_{j=1}^{n} \nabla^{i} V\left(y_{t}\right) B_{i, j}^{n}(t) \mathrm{d} g_{j}(t), \\
\mathcal{D}_{g_{1}, \ldots, g_{n}}^{n} y_{0}= & 0,
\end{aligned}
$$

where $A_{i}^{n}$ and $B_{i, j}^{n}$ are respectively defined by (51) and (52).

REMARK 3.3. The symmetry of the higher-order derivatives of $V$ ensures that we may simplify $\nabla^{i} V\left(y_{t}\right) A_{i}^{n}(t)$ in (51) by replacing the symmetric tensor product with the usual tensor product to give

$$
\nabla^{i} V\left(y_{t}\right) A_{i}^{n}(t)=\sum_{\pi=\left\{\pi_{1}, \ldots, \pi_{i}\right\} \in \mathcal{P}\left(\left\{g_{1}, \ldots, g_{n}\right\}\right)} \nabla^{i} V\left(y_{t}\right) \mathcal{D}_{\pi_{1}}^{\left|\pi_{1}\right|} y_{t} \otimes \cdots \otimes \mathcal{D}_{\pi_{i}}^{\left|\pi_{i}\right|} y_{t} .
$$

The terms $\nabla^{i} V\left(y_{t}\right) B_{i, j}^{n}(t)$ may also be simplified similarly. For this reason it is sufficient to prove (54) for paths $A_{i}^{n}$ and $B_{i, j}^{n}$ whose symmetrizations coincide with the right-hand sides of (51) and (52), respectively.

PROOF. We begin with the case $n=2$. Taking the directional derivative of $\mathcal{D}_{g_{1}} y_{t}$ [see (41)] in the direction of $g_{2}$, we see that $\mathcal{D}_{g_{1}, g_{2}}^{2} y_{t}$ solves the RDE

$$
\begin{aligned}
\mathrm{d} \mathcal{D}_{g_{1}, g_{2}}^{2} y_{t}= & \nabla V\left(y_{t}\right)\left(\mathcal{D}_{g_{1}, g_{2}}^{2} y_{t}\right) \circ \mathrm{d} \mathbf{x}_{t}+\nabla^{2} V\left(y_{t}\right)\left(\mathcal{D}_{g_{1}} y_{t}, \mathcal{D}_{g_{2}} y_{t}\right) \circ \mathrm{d} \mathbf{x}_{t} \\
& +\nabla V\left(y_{t}\right)\left(\mathcal{D}_{g_{2}} y_{t}\right) \mathrm{d} g_{1}(t)+\nabla V\left(y_{t}\right)\left(\mathcal{D}_{g_{1}} y_{t}\right) \mathrm{d} g_{2}(t) .
\end{aligned}
$$

The proof is completed by induction. Assuming (54) is true for $n=2, \ldots, k$, one can take the directional derivative of $\mathcal{D}_{g_{1}, \ldots, g_{k}} y_{t}$ in the direction $g_{k+1}$ to obtain the identity

$$
\begin{aligned}
\mathcal{D}_{g_{1}, \ldots, g_{k+1}}^{k+1} y_{t}= & \sum_{i=1}^{k} \mathcal{D}_{g_{k+1}} \int_{0}^{t} \nabla^{i} V\left(y_{s}\right) A_{i}^{k}(s) \circ \mathrm{d} \mathbf{x}_{s} \\
& +\sum_{i=1}^{k-1} \sum_{j=1}^{k} \mathcal{D}_{g_{k+1}} \int_{0}^{t} \nabla^{i} V\left(y_{s}\right) B_{i, j}^{k}(s) \mathrm{d} g_{j}(s)
\end{aligned}
$$




$$
\begin{aligned}
= & \sum_{i=1}^{k+1} \int_{0}^{t} \nabla^{i} V\left(y_{s}\right) \tilde{A}_{i}^{k+1}(s) \circ \mathrm{d} \mathbf{x}_{s} \\
& +\sum_{i=1}^{k} \sum_{j=1}^{k+1} \int_{0}^{t} \nabla^{i} V\left(y_{s}\right) \tilde{B}_{i, j}^{k+1}(s) \mathrm{d} g_{j}(s),
\end{aligned}
$$

where the coefficients $\tilde{A}_{i}^{k+1}$ and $\tilde{B}_{i}^{k+1}$ are the $\left(\mathbb{R}^{e}\right)^{\otimes i}$-valued paths defined for $t \in$ $[0, T]$ by

$$
\tilde{A}_{i}^{k+1}(t)= \begin{cases}\mathcal{D}_{g_{k+1}} A_{1}^{k}(t), & i=1, \\ \mathcal{D}_{g_{k+1}} A_{i}^{k}(t)+A_{i-1}^{k}(t) \otimes \mathcal{D}_{g_{k+1}} y_{t}, & i=2, \ldots, k, \\ A_{k}^{k}(t) \otimes \mathcal{D}_{g_{k+1}} y_{t}, & i=k+1,\end{cases}
$$

and

$$
\tilde{B}_{i, j}^{k+1}(t)= \begin{cases}\mathcal{D}_{g_{k+1}} B_{1, j}^{k}(t), & i=1, j=1, \ldots, k, \\ \mathcal{D}_{g_{k+1}} B_{i, j}^{k}(t) & \\ \quad+B_{i-1, j}^{k}(t) \otimes \mathcal{D}_{g_{k+1}} y_{t}, & i=2, \ldots, k-1, j=1, \ldots, k, \\ B_{i-1, j}^{k}(t) \otimes \mathcal{D}_{g_{k+1}} y_{t}, & i=k, j=1, \ldots, k, \\ A_{i}^{k}(t), & i=1, \ldots, k, j=k+1 .\end{cases}
$$

To finish the inductive step, we first show that for every $t \in[0, T]$,

$$
\tilde{A}_{i}^{k+1}(t) \stackrel{=}{=} A_{i}^{k+1}(t) \quad \forall i=1, \ldots, k+1,
$$

where $a \tilde{=} b$ means that the symmetrizations of the tensors $a$ and $b$ are equal. From this, it immediately follows that $\nabla^{i} V\left(y_{t}\right) \tilde{A}_{i}^{k+1}(t)=\nabla^{i} V\left(y_{t}\right) A_{i}^{k+1}(t)$ for all $i=$ $1, \ldots, k+1$. We check (58) for the boundary cases first. For $i=1$, the induction hypothesis gives at once that

$$
\tilde{A}_{1}^{k+1}(t)=\mathcal{D}_{g_{1}, \ldots, g_{k+1}}^{k+1} y_{t},
$$

whereas the case $i=k+1$ follows from

$$
\begin{aligned}
\tilde{A}_{k+1}^{k+1}(t) & =A_{k}^{k}(t) \otimes \mathcal{D}_{g_{k+1}} y_{t} \\
& \tilde{=} A_{k}^{k}(t) \tilde{\otimes} \mathcal{D}_{g_{k+1}} y_{t} \\
& =\mathcal{D}_{g_{1}} y_{t} \tilde{\otimes} \cdots \tilde{\otimes} \mathcal{D}_{g_{k+1}} y_{t}=A_{k+1}^{k+1}(t) .
\end{aligned}
$$

For the remaining cases $i=2, \ldots, k$, we note that any partition of $\left\{g_{1}, \ldots, g_{k+1}\right\}$ of size $i$ can be formed from a partition $\pi$ of $\left\{g_{1}, \ldots, g_{k}\right\}$ in one of two ways. The first way is that $\pi=\left\{\pi_{1}, \ldots, \pi_{i}\right\}$ itself has size $i$ and $g_{k}$ is assigned to one of the subsets $\pi_{1}, \ldots, \pi_{i}$. The second way is that $\pi=\left\{\pi_{1}, \ldots, \pi_{i-1}\right\}$ has size $i-1$ and $\left\{g_{k+1}\right\}$ is adjoined as a singleton to give $\left\{\pi_{1}, \ldots, \pi_{i-1},\left\{g_{k+1}\right\}\right\}$. The two terms in (56) obtained by differentiation and the tensor product respectively correspond to 
these operations. By the induction hypothesis, $A_{i}^{k}$ (resp., $A_{i-1}^{k}$ ) includes a summation over all partitions of $\left\{g_{1}, \ldots, g_{n}\right\}$ of size $i$ (resp., $\left.i-1\right)$, thus every partition of $\left\{g_{1}, \ldots, g_{k+1}\right\}$ of size $i$ is accounted for in (56). It follows immediately that

$$
\tilde{A}_{i}^{k+1}(t) \stackrel{\sim}{=} A_{i}^{k+1}(t) .
$$

Finally, we show that for every $t \in[0, T]$,

$$
\tilde{B}_{i, j}^{k+1}(t) \stackrel{\sim}{=} B_{i, j}^{k+1}(t) \quad \forall i=1, \ldots, k, j=1, \ldots, k+1 .
$$

Again we treat the boundary cases separately. For $j=k+1$, from the definition of $\tilde{B}$ and $A$, we have

$$
\tilde{B}_{i, k+1}^{k+1}(t)=A_{i}^{k}(t)=B_{i, k+1}^{k+1}(t) \quad \forall i=1, \ldots, k .
$$

For $i=1$, we have $\forall j=1, \ldots, k$,

$$
\begin{aligned}
\tilde{B}_{1, j}^{k+1}(t) & =\mathcal{D}_{g_{k+1}} B_{1, j}^{k}(t) \\
& =\mathcal{D}_{g_{k+1}} \mathcal{D}_{g_{1}, \ldots, g_{j-1}, g_{j+1}, \ldots, g_{k}}^{k-1} y_{t} \\
& =\mathcal{D}_{g_{1}, \ldots, g_{j-1}, g_{j+1}, \ldots, g_{k+1}}^{k} y_{t}=B_{1, j}^{k+1}(t) .
\end{aligned}
$$

The remaining terms are dealt with by exactly the same argument used for the nonboundary $\tilde{A}$ terms, and the induction is thereby complete.

The following corollary makes explicit the expression given in Proposition 11.5 of [18].

COROLLARY 3.4. Under the conditions of the preceding theorem,

$$
\begin{aligned}
\mathcal{D}_{g_{1}, \ldots, g_{n}}^{n} y_{t}= & \sum_{i=2}^{n} \int_{0}^{t} J_{t}^{\mathbf{x}}\left(J_{s}^{\mathbf{x}}\right)^{-1} \nabla^{i} V\left(y_{s}\right) A_{i}^{n}(s) \circ \mathrm{d} \mathbf{x}_{s} \\
& +\sum_{i=1}^{n-1} \sum_{j=1}^{n} \int_{0}^{t} J_{t}^{\mathbf{x}}\left(J_{s}^{\mathbf{x}}\right)^{-1} \nabla^{i} V\left(y_{s}\right) B_{i, j}^{n}(s) \mathrm{d} g_{j}(s)
\end{aligned}
$$

for all $n \geq 2$.

PROOF. From formula (54) and the fact that

$$
A_{1}^{n}(s)=\mathcal{D}_{g_{1}, \ldots, g_{n}}^{n} y_{s},
$$

(59) can be recovered using Duhamel's principle.

We now arrive at the main result of this section. 
Proposition 3.5. Let $p \in[2,3), q:=\frac{p}{2}$ and $n \in \mathbb{N}$. Let $y$ be the solution to the $R D E$

$$
\mathrm{d} y_{t}=V\left(y_{t}\right) \circ \mathrm{d} \mathbf{x}_{t}, \quad y_{0} \in \mathbb{R}^{e} \text { given, }
$$

where $\mathbf{x} \in \mathcal{C}^{0, p \text {-var }}\left([0, T] ; G^{2}\left(\mathbb{R}^{d}\right)\right)$ and $V \in \mathcal{C}_{b}^{2+n}\left(\mathbb{R}^{e} ; \mathbb{R}^{e} \otimes \mathbb{R}^{d}\right)$. Then there exists a polynomial $P_{d(n)}: \mathbb{R}_{+} \times \mathbb{R}_{+} \rightarrow \mathbb{R}_{+}$of finite degree $d(n)$ for which

$$
\begin{aligned}
& \left\|\mathcal{D}_{g_{1}, \ldots, g_{n}}^{n} y \cdot\right\|_{\mathcal{V} p ;[0, T]} \\
& \quad \leq P_{d(n)}\left(\|\mathbf{x}\|_{p \text {-var; }[0, T]}, \exp \left(C N_{1 ;[0, T]}^{\mathbf{x}}\right)\right) \prod_{i=1}^{n}\left\|g_{i}\right\|_{q \text {-var; }[0, T]},
\end{aligned}
$$

for any $g_{1}, \ldots, g_{n} \in C^{q-\operatorname{var}}\left([0, T] ; \mathbb{R}^{d}\right)$. Here, $N_{1}^{\mathbf{x}}$ is defined as in (44), and the constant $C$ as well as the coefficients of $P_{d(n)}$ depend only on $\|V\|_{\mathcal{C}_{b}^{2+n}}$ and $p$.

PROOF. The proof will proceed by induction. First, we denote

$$
F_{t}^{i}:=\left(J_{t}^{\mathbf{x}}\right)^{-1} \nabla^{i} V\left(y_{t}\right), \quad i=0,1, \ldots
$$

Applying Theorem 3.1 together with Proposition 2.21 to $\nabla^{i} V(y)$, we see that there exists an integer $k_{1}$ such that

$$
\left\|\nabla^{i} V(y)\right\|_{p-\mathrm{cvar}} \leq C_{1}\left(1+\|\mathbf{x}\|_{p \text {-var; }[0, T]}\right)^{k_{1}}
$$

[note from (15) that $\left\|\mathbf{x}^{k}\right\| \frac{p}{k}$-var; [0,T] $\leq C\|\mathbf{x}\|_{p \text {-var;[0,T] }}^{k}$ for all $\left.k\right]$ and again from Theorem 3.1, we know that there exist $k_{2}$ and $l$ such that

$$
\begin{aligned}
& \left\|J^{\mathbf{x}}\right\|_{p \text {-cvar }},\left\|\left(J^{\mathbf{x}}\right)^{-1}\right\|_{p \text {-cvar }} \\
& \quad \leq C_{2}\left(1+\exp \left(C_{3} N_{1 ;[0, T]}^{\mathbf{x}}\right)\right)^{k_{2}}\left(1+\|\mathbf{x}\|_{p \text {-var; }[0, T]}\right)^{l} .
\end{aligned}
$$

Now applying Leibniz rule, that is, Proposition 2.22, we have, for some integer $k$,

$$
\left\|F^{i}\right\|_{p \text {-cvar }} \leq C_{1}\left(1+\exp \left(C_{2} N_{1 ;[0, T]}^{\mathbf{x}}\right)\right)^{k}\left(1+\|\mathbf{x}\|_{p \text {-var; }[0, T]}\right)^{l},
$$

where $C_{1}$ and $C_{2}$ depend only on $p$ and $\|V\|_{\mathcal{C}_{b}^{2+i}}$.

We now begin with the base case $n=1$. Let $\phi_{t}$ denote $J_{t}^{\mathbf{x}}$ and $\psi_{t}$ denote $\int_{0}^{t}\left(J_{s}^{\mathbf{x}}\right)^{-1} V\left(y_{s}\right) \mathrm{d} g(s)$. Then $\mathcal{D}_{g} y_{t}=\phi_{t} \psi_{t}$ and applying Young's inequality to $\psi_{t}$, we obtain

$$
\begin{aligned}
& \left\|\int_{0}^{t}\left(J_{s}^{\mathbf{x}}\right)^{-1} V\left(y_{s}\right) \mathrm{d} g(s)\right\|_{q \text {-var; }[0, T]} \\
& \quad \leq C_{p}\left\|\left(J^{\mathbf{x}}\right)^{-1} V(y)\right\|_{\mathcal{V}^{p} ;[0, T]}\|g\|_{q \text {-var; }[0, T]} .
\end{aligned}
$$

Continuing, the second part of Proposition 2.22 tells us that $\mathcal{D}_{g} y$ is controlled by $\mathbf{x}$, and from the bounds (63) and (62), we have

$$
\left\|\mathcal{D}_{g} y \cdot\right\|_{p-\mathrm{cvar}} \leq C_{3}\left(1+\exp \left(C N_{1 ;[0, T]}^{\mathbf{x}}\right)\right)^{k}\left(1+\|\mathbf{x}\|_{p \text {-var; }[0, T]}\right)^{l}\|g\|_{q-\mathrm{var} ;[0, T]}
$$


The cases $n \geq 2$ are proved in the same manner. Let $z_{t}^{n}$ denote

$$
z_{t}^{n}:=\mathcal{D}_{g_{1}, \ldots, g_{n}}^{n} y_{t}
$$

where $\left\{g_{i}\right\}_{i=1}^{n}$ is an arbitrary subset of $C^{q-v a r}\left([0, T] ; \mathbb{R}^{d}\right)$, and the induction hypothesis is as follows:

Assume that for all $n=1, \ldots, k, z^{n}$ is controlled by $\mathbf{x}$, and that

$$
\left\|z^{n}\right\|_{p-\mathrm{cvar}} \leq P_{d(n)}\left(\|\mathbf{x}\|_{p-\operatorname{var} ;[0, T]}, \exp \left(C N_{1 ;[0, T]}^{\mathbf{x}}\right)\right) \prod_{i=1}^{n}\left\|g_{i}\right\|_{q-\operatorname{var} ;[0, T]} .
$$

To show the result for $n=k+1$, first recall from Theorem 3.2 that $z_{t}^{k+1}=$ $\mathcal{D}_{g_{1}, \ldots, g_{k+1}}^{k+1} y_{t}$ equals

$$
J_{t}^{\mathbf{x}}\left(H_{t}^{k+1}+G_{t}^{k+1}\right)
$$

where

$$
H_{t}^{k+1}:=\int_{0}^{t} \sum_{i=2}^{k+1} F_{s}^{i} A_{i}^{k+1}(s) \circ \mathrm{d} \mathbf{x}_{s}
$$

and

$$
G_{t}^{k+1}:=\int_{0}^{t} \sum_{i=1}^{k} \sum_{j=1}^{k+1} F_{s}^{i} B_{i, j}^{k+1}(s) \mathrm{d} g_{j}(s) .
$$

From the induction hypothesis and Leibniz rule, for a partition $\pi=\left\{\pi_{1}, \ldots, \pi_{i}\right\}$ in $\mathcal{P}\left(\left\{g_{1}, \ldots, g_{k+1}\right\}\right)$, we have the bound

$$
\left\|\mathcal{D}_{\pi_{1}}^{\left|\pi_{1}\right|} y \cdot \tilde{\otimes} \cdots \tilde{\otimes} \mathcal{D}_{\pi_{i}}^{\left|\pi_{i}\right|} y \cdot\right\|_{p \text {-cvar }}
$$

$$
\leq \prod_{l=1}^{i} P_{d\left(\left|\pi_{l}\right|\right)}\left(\|\mathbf{x}\|_{p-\operatorname{var} ;[0, T]}, \exp \left(C N_{1 ;[0, T]}^{\mathbf{x}}\right)\right) \prod_{m=1}^{k+1}\left\|g_{m}\right\|_{q-\operatorname{var} ;[0, T]} .
$$

Similarly, for a partition $\pi=\left\{\pi_{1}, \ldots, \pi_{i}\right\} \in \mathcal{P}\left(\left\{g_{1}, \ldots, g_{j-1}, g_{j+1}, \ldots, g_{k+1}\right\}\right)$ we have the bound

$$
\begin{aligned}
& \left\|\mathcal{D}_{\pi_{1}}^{\left|\pi_{1}\right|} y \cdot \tilde{\otimes} \cdots \tilde{\otimes} \mathcal{D}_{\pi_{i}}^{\left|\pi_{i}\right|} y \cdot\right\|_{p \text {-cvar }} \\
& \quad \leq \prod_{l=1}^{i} P_{d\left(\left|\pi_{l}\right|\right)}\left(\|\mathbf{x}\|_{p \text {-var; }[0, T]}, \exp \left(C N_{1 ;[0, T]}^{\mathbf{x}}\right)\right) \prod_{m=1, m \neq j}^{k+1}\left\|g_{m}\right\|_{q \text {-var; }[0, T]} .
\end{aligned}
$$

Recalling the definition of $A_{i}^{k+1}(s)$ in (51), we use (66) together with bound (63) and apply Leibniz rule. After summing over $i$ and invoking Theorem 2.20, we see that $H^{k+1}$ is controlled by $\mathbf{x}$, and there exists a polynomial $\tilde{P}_{1}$ such that

$$
\left\|H^{k+1}\right\|_{p \text {-cvar }} \leq \tilde{P}_{1}\left(\|\mathbf{x}\|_{p \text {-var; }[0, T]}, \exp \left(C N_{1 ;[0, T]}^{\mathbf{x}}\right)\right) \prod_{i=1}^{k+1}\left\|g_{i}\right\|_{q \text {-var; }[0, T]} .
$$


For $G^{k+1}$, we will show that its $q$-variation is bounded similarly, and then add it to the remainder term of $H^{k+1}$ to make $H^{k+1}+G^{k+1}$ a controlled rough path (with the appropriate bounds). Fixing $i$ and $j$, from Young's inequality we have

$$
\begin{aligned}
& \left\|\int_{0} F_{s}^{i} B_{i, j}^{k+1}(s) \mathrm{d} g_{j}(s)\right\|_{q \text {-var;[0,T] }} \\
& \quad \leq C_{p}\left\|F^{i}\right\|_{\mathcal{V}^{p} ;[0, T]}\left\|B_{i, j}^{k+1}\right\|_{\mathcal{V}^{p} ;[0, T]}\left\|g_{j}\right\|_{q \text {-var. }}
\end{aligned}
$$

Now if we recall the definition of $B_{i, j}^{k+1}(s)$ in (52) and use (67) in the above expression, after summing over all $i$ and $j$, we obtain some polynomial $\tilde{P}_{2}$ such that

$$
\left\|G^{k+1}\right\|_{q-\operatorname{var} ;[0, T]} \leq \tilde{P}_{2}\left(\|\mathbf{x}\|_{p-\operatorname{var} ;[0, T]}, \exp \left(C N_{1 ;[0, T]}^{\mathbf{x}}\right)\right) \prod_{i=1}^{k+1}\left\|g_{i}\right\|_{q-\operatorname{var} ;[0, T]} .
$$

Finally, applying Leibniz rule to $z_{t}^{k+1}=J_{t}^{\mathbf{x}}\left(H_{t}^{k+1}+G_{t}^{k+1}\right)$ shows us that $z^{k+1}$ is controlled by $\mathbf{x}$ with the bound

$$
\left\|z^{k+1}\right\|_{p \text {-cvar }} \leq P_{d(k+1)}\left(\|\mathbf{x}\|_{p-\operatorname{var} ;[0, T]}, \exp \left(C N_{1 ;[0, T]}^{\mathbf{x}}\right)\right) \prod_{i=1}^{k+1}\left\|g_{i}\right\|_{q-\operatorname{var} ;[0, T]}
$$

REMARK 3.6. Our main use of this result will be in the stochastic setting where $\mathbf{X}$ is a Gaussian rough path zero mean continuous Gaussian process with i.i.d. components, and a covariance function $R$ of finite $2 \mathrm{D} \rho$-variation for some $\rho \in\left[1, \frac{3}{2}\right)$. Under these assumptions, Proposition 3.5 can be applied with $g_{i}:=$ $\Phi\left(h_{i}\right)$ for any collection $\left\{h_{i}\right\} \subset \mathcal{H}_{1}^{d}$. Note also in this section we abuse the notation by using $\mathcal{D}_{g_{i}} F$ rather than $\mathbf{D}_{g_{i}} F$ (see Remark 2.16); in later sections, the subscript will be elements in $\mathcal{H}_{1}^{d}$ rather than $\mathcal{H}^{d}$.

4. An isomorphism and dense subspace of the Cameron-Martin space. In this section, we will identify a dense subspace of the Cameron-Martin space which will be of importance later. The motivation is as follows: let $Y$ be a solution to RDE (39). We would like to show that $Y \in \mathbb{D}^{1,2}\left(\mathcal{H}_{1}^{d}\right)$, which in turn implies that $Y$ is Skorohod integrable. To do so, consider a partition $\pi=\left\{r_{i}\right\}$ of $[0, T]$, and observe that

$$
Y^{\pi}(t):=\sum_{i} Y_{r_{i}} \mathbb{1}_{\left[r_{i}, r_{i+1}\right)}(t)
$$

is almost surely an element of $\mathcal{H}_{1}^{d}$. Using Itô-Skorohod isometry, we have

$$
\mathbb{E}\left[\delta^{X}\left(Y^{\pi}-Y\right)^{2}\right] \leq \mathbb{E}\left[\left\|Y^{\pi}-Y\right\|_{\mathcal{H}_{1}^{d}}^{2}\right]+\mathbb{E}\left[\left\|\mathcal{D} Y^{\pi}-\mathcal{D} Y\right\|_{\mathcal{H}_{1}^{d} \otimes \mathcal{H}_{1}^{d}}^{2}\right] .
$$

Thus if we can show that almost surely, $\left\|Y^{\pi}-Y\right\|_{\mathcal{H}_{1}^{d}}$ and $\left\|\mathcal{D} Y^{\pi}-\mathcal{D} Y\right\|_{\mathcal{H}_{1}^{d} \otimes \mathcal{H}_{1}^{d}}$ both vanish as $\|\pi\| \rightarrow 0$, then with further integrability assumptions one can apply dominated convergence to show that $\delta^{X}\left(Y^{\pi}\right)$ converges to $\delta^{X}(Y)$ in $L^{2}(\Omega)$. 
We will investigate the (almost sure) regularity required of $Y$ to identify it as an element of $\mathcal{H}_{1}^{d}$ and to have $\left\|Y^{\pi}-Y\right\|_{\mathcal{H}_{1}^{d}} \rightarrow 0$. We first note the following lemma, which is a direct consequence of Theorem 2.12.

LEMMA 4.1. Let $f \in \mathcal{C}_{\mathrm{pw}}^{p \text {-var }}\left([0, T] ; \mathbb{R}^{e}\right)$ and $R$ be continuous and of finite $\rho$-variation where we assume that $\frac{1}{p}+\frac{1}{\rho}>1$. For any partition $\pi=\left\{r_{i}\right\}$ of $[0, T]$, let $f^{\pi}$ denote

$$
f^{\pi}(t):=\sum_{i} f\left(r_{i}\right) \mathbb{1}_{\left[r_{i}, r_{i+1}\right)}(t)
$$

Then

$$
\lim _{\|\pi\| \rightarrow 0}\left|\int_{[0, T]^{2}}\left\langle f_{s}^{\pi}-f_{s}, f_{t}^{\pi}-f_{t}\right\rangle_{\mathbb{R}^{e}} \mathrm{~d} R(s, t)\right|=0 .
$$

ProOF. Since $f \in \mathcal{C}_{\mathrm{pw}}^{p \text {-var }}\left([0, T] ; \mathbb{R}^{e}\right)$, we can partition $[0, T]^{2}$ into finite subregions $\left[x_{i}, x_{i+1}\right] \times\left[x_{j}, x_{j+1}\right]$, where $\left\langle f_{s}, f_{t}\right\rangle_{\mathbb{R}^{e}}$ is continuous in the interior of each such subregion. We will show (69) in each subregion, and the case for $[0, T]^{2}$ will follow from summing the Young integral over all the subregions.

By assumption $f$ has finite $p$-variation over $[0, T]$ and it is therefore a regulated function, that is, it has finite right and left limits everywhere in $(0, T)$ and a finite right (resp., left) limit at the left (resp., right) end point. Hence, for all $i$, there exists a unique continuous function $F_{i}:\left[x_{i}, x_{i+1}\right] \rightarrow \mathbb{R}^{e}$ which agrees with $f$ on $\left(x_{i}, x_{i+1}\right)$.

To avoid the discontinuities on the boundary, we will bound the integral over the subset $A_{i, j}^{\pi}:=\left[r_{\bar{k}(i)}, r_{\underline{k}(i+1)}\right] \times\left[r_{\bar{k}(j)}, r_{\underline{k}(j+1)}\right]$ of $A_{i, j}:=\left[x_{i}, x_{i+1}\right] \times\left[x_{j}, x_{j+1}\right]$, where

$$
r_{\bar{k}(l)}:=\inf \left\{r_{k} \mid r_{k}>x_{l}\right\}, \quad r_{\underline{k}(l)}:=\sup \left\{r_{k} \mid r_{k}<x_{l}\right\} .
$$

From Young's inequality (24) and the fact that $\omega_{R, \rho+\varepsilon}$ is a control, the integral over the region $A_{i, j}-A_{i, j}^{\pi}$ will be arbitrarily small as $\|\pi\|$ tends to zero. Let $\omega_{i, j}$ denote the control

$$
\omega_{i, j}([s, t] \times[u, v]):=\left\|F_{i}\right\|_{p \text {-var; }[s, t]}^{p}\left\|F_{j}\right\|_{p \text {-var; }[u, v]}^{p}, \quad(s, t) \times(u, v) \in A_{i, j} .
$$

Continuing,

$$
\omega([s, t] \times[u, v]):=\omega_{i, j}^{\frac{1}{\theta p}}([s, t] \times[u, v]) \omega_{R, \rho+\varepsilon}^{\frac{1}{\theta(\rho+\varepsilon)}}([s, t] \times[u, v]),
$$

is again a control, where $\varepsilon$ is chosen such that $\theta:=\frac{1}{p}+\frac{1}{\rho+\varepsilon}>1$.

Now we have

$$
\begin{aligned}
& \quad\left|\int_{A_{i, j}^{\pi}}\left\langle f_{s}^{\pi}-f_{s}, f_{t}^{\pi}-f_{t}\right\rangle_{\mathbb{R}^{e}} \mathrm{~d} R(s, t)\right| \\
& \quad \leq \sum_{k, l}\left|\int_{r_{k}}^{r_{k+1}} \int_{r_{l}}^{r_{l+1}}\left\langle f_{s}-f_{r_{k}}, f_{t}-f_{r_{l}}\right\rangle_{\mathbb{R}^{e}} \mathrm{~d} R(s, t)\right|,
\end{aligned}
$$


where the sum is taken over the partition points in $A_{i, j}^{\pi}$. By Young's inequality (24) and the fact that $\left\langle f_{s}-f_{r_{k}}, f_{t}-f_{r_{l}}\right\rangle_{\mathbb{R}^{e}}$ vanishes at all points $\left(r_{k}, r_{l}\right)$ in $A_{i, j}^{\pi}$, the expression above is bounded by

$$
\begin{aligned}
C_{p, \rho} & \sum_{k, l}\left\|F_{i}\right\|_{p-\operatorname{var} ;\left[r_{k}, r_{k+1}\right]}\left\|F_{j}\right\|_{p-\operatorname{var} ;\left[r_{l}, r_{l+1}\right]}\|R\|_{\rho+\varepsilon-\operatorname{var} ;\left[r_{k}, r_{k+1}\right] \times\left[r_{l}, r_{l+1}\right]} \\
& \leq C_{p, \rho} \sum_{k, l} \omega^{\theta}\left(\left[r_{k}, r_{k+1}\right] \times\left[r_{l}, r_{l+1}\right]\right) \\
& \leq C_{p, \rho} \max _{k, l} \omega^{\theta-1}\left(\left[r_{k}, r_{k+1}\right] \times\left[r_{l}, r_{l+1}\right]\right) \omega\left(A_{i, j}\right),
\end{aligned}
$$

which tends to zero as the mesh of the partition goes to zero.

4.1. A dense subspace of $\mathcal{H}_{1}^{d}$. We now give a novel characterization of a subspace of $\mathcal{H}_{1}^{d}$ using Young-Stieltjes integrals. Let $R$ be of finite 2D $\rho$-variation, where $\rho \in[1,2)$. We define

$$
\mathcal{W}_{\rho}^{d}:=\bigcup_{p<\frac{\rho}{\rho-1}} \mathcal{C}_{\mathrm{pw}}^{p-\operatorname{var}}\left([0, T] ; \mathbb{R}^{d}\right)
$$

and equip it with the inner product

$$
\langle f, g\rangle_{\mathcal{W}_{\rho}^{d}}:=\int_{[0, T]^{2}}\left\langle f_{s}, g_{t}\right\rangle_{\mathbb{R}^{d}} \mathrm{~d} R(s, t) .
$$

One can check that $\langle\cdot, \cdot\rangle_{\mathcal{W}_{\rho}^{d}}$ defines a semi-inner product; it is bilinear due to the linearity of the Young-Stieltjes integral, and positive semi-definite as well as symmetric because the covariance function $R$ is positive semi-definite and symmetric. We will identify $f$ and $g$ to be in the same equivalence class if $\langle f-g, f-g\rangle_{\mathcal{W}_{\rho}^{d}}=0$, and quotient $\mathcal{W}_{\rho}^{d}$ with respect to these classes. This then makes $\langle\cdot, \cdot\rangle_{\mathcal{W}_{\rho}^{d}}$ a proper inner product.

Proposition 4.2. $\quad \mathcal{W}_{\rho}^{d}$ is a dense subspace of $\mathcal{H}_{1}^{d}$, and the inclusion map $i:\left(\mathcal{W}_{\rho}^{d},\langle\cdot, \cdot\rangle_{\mathcal{W}_{\rho}^{d}}\right) \rightarrow\left(\mathcal{H}_{1}^{d},\langle\cdot, \cdot\rangle_{\mathcal{H}_{1}^{d}}\right)$ is an isometry.

ProOF. Let $f \in \mathcal{W}_{\rho}^{d}$ and let $\pi(n)=\left\{r_{i}^{(n)}\right\}$ be a sequence of partitions whose mesh vanishes as $n \rightarrow \infty$. As usual, we denote

$$
f^{\pi(n)}:=\sum_{i} f\left(r_{i}^{(n)}\right) \mathbb{1}_{\left[r_{i}^{(n)}, r_{i+1}^{(n)}\right)}(t) .
$$


Now the key point to note is that for each $n, f^{\pi(n)}$ is in $\mathcal{W}_{\rho}^{d} \cap \mathcal{H}_{1}^{d}$; moreover,

$$
\begin{aligned}
\left\|f^{\pi(n)}\right\|_{\mathcal{H}_{1}^{d}}^{2} & =\sum_{i, j}\left\langle f_{r_{i}^{(n)}}, f_{r_{j}^{(n)}}\right\rangle_{\mathbb{R}^{d}}\left\langle\mathbb{1}_{\left[r_{i}^{(n)}, r_{i+1}^{(n)}\right)}, \mathbb{1}_{\left[r_{j}^{(n)}, r_{j+1}^{(n)}\right)}\right\rangle \mathcal{H}_{1} \\
& =\sum_{i, j}\left\langle f_{r_{i}^{(n)}}, f_{r_{j}^{(n)}}\right\rangle_{\mathbb{R}^{d}} R\left(\begin{array}{ll}
r_{i}^{(n)} & r_{i+1}^{(n)} \\
r_{j}^{(n)} & r_{j+1}^{(n)}
\end{array}\right) \\
& =\left\|f^{\pi(n)}\right\|_{\mathcal{W}_{\rho}^{d}}^{2} .
\end{aligned}
$$

From Lemma 4.1, $\left\|f^{\pi(n)}-f\right\|_{\mathcal{W}_{\rho}^{d}} \rightarrow 0$, which means that $f^{\pi(n)}$ is Cauchy and from (72) and the completeness of $\mathcal{H}_{1}^{d}, \lim _{n \rightarrow \infty} f^{\pi(n)}$ exists in $\mathcal{H}_{1}^{d}$. We identify $f$ with this limit and under this identification we have

$$
\|f\|_{\mathcal{H}_{1}^{d}}^{2}=\int_{[0, T]^{2}}\left\langle f_{s}, f_{t}\right\rangle_{\mathbb{R}^{d}} \mathrm{~d} R(s, t) .
$$

Since $\mathcal{W}_{\rho}^{d}$ contains all the generating functions $\left\{\mathbb{1}_{[0, t)}^{(u)}(\cdot)\right\}$ of $\mathcal{H}_{1}^{d}$, its completion, and hence closure, is all of $\mathcal{H}_{1}^{d}$.

REMARK 4.3. We recall the following nondegeneracy condition on Gaussian processes which is featured in [7]. We say that $R$ (or equivalently, $X$ ) is nondegenerate on $[0, T]$ if the following implication holds:

$$
\int_{[0, T]^{2}}\left\langle f_{s}, f_{t}\right\rangle_{\mathbb{R}^{d}} \mathrm{~d} R(s, t)=0 \Rightarrow f=0 \quad \text { a.e. }
$$

Under this condition, each equivalence class of $\mathcal{W}_{\rho}^{d}$ would then consist of functions which agree almost everywhere.

4.2. The Malliavin derivative and convergence in the tensor norm. We will now extend the results of the last section to the tensor space $\mathcal{H}_{1}^{d} \otimes \mathcal{H}_{1}^{d}$. Let $\left.\left.\mathbf{X} \in \mathcal{C}^{0, p-\operatorname{var}}([0, T] ; G\rfloor p\right\rfloor\left(\mathbb{R}^{d}\right)\right)$ and assume that for all $h \in \mathcal{H}_{1}^{d}, \Phi(h)$ can be embedded in $\mathcal{C}^{q-\operatorname{var}}\left([0, T] ; \mathbb{R}^{d}\right)$ where $\frac{1}{p}+\frac{1}{q}>1$. Then the Malliavin derivative of $Y$ satisfying

$$
\mathrm{d} Y_{t}=V\left(Y_{t}\right) \circ \mathrm{d} \mathbf{X}_{t}, \quad Y_{0}=y_{0},
$$

is given by

$$
\begin{aligned}
\mathcal{D}_{h} Y_{t} & =\int_{0}^{t} J_{t}^{\mathbf{X}}\left(J_{s}^{\mathbf{X}}\right)^{-1} V\left(Y_{s}\right) \mathrm{d} \Phi(h)(s) \\
& =\int_{0}^{T} \mathbb{1}_{[0, t)}(s) J_{t}^{\mathbf{X}}\left(J_{s}^{\mathbf{X}}\right)^{-1} V\left(Y_{s}\right) \mathrm{d} \Phi(h)(s) .
\end{aligned}
$$


Using $g(s, t)$ to denote the Malliavin derivative,

$$
g(s, t):=\mathcal{D}_{s} Y_{t}=\mathbb{1}_{[0, t)}(s) J_{t}^{\mathbf{X}}\left(J_{s}^{\mathbf{X}}\right)^{-1} V\left(Y_{s}\right),
$$

then with respect to any partition $\pi=\left\{r_{i}\right\}$ of $[0, T]$, we also have

$$
\begin{aligned}
\mathcal{D}_{s} Y_{t}^{\pi} & =\sum_{i} \mathcal{D}_{s} Y_{r_{i}} \mathbb{1}_{\left[r_{i}, r_{i+1}\right)}(t) \\
& =\sum_{i} g\left(s, r_{i}\right) \mathbb{1}_{\left[r_{i}, r_{i+1}\right)}(t) .
\end{aligned}
$$

We will proceed to show that $\mathcal{D} Y^{\pi}$ lies in $\mathcal{H}_{1}^{d} \otimes \mathcal{H}_{1}^{d}$ almost surely, and under suitable regularity assumptions on $\mathcal{D} Y$, we have $\left\|\mathcal{D} Y^{\pi}-\mathcal{D} Y\right\|_{\mathcal{H}_{1}^{d} \otimes \mathcal{H}_{1}^{d}} \rightarrow 0$ as $\|\pi\| \rightarrow 0$. Coupled with the results in the previous section, this will mean that $Y^{\pi}$ converges to $Y$ in $\mathbb{D}^{1,2}\left(\mathcal{H}_{1}^{d}\right)$, and $\delta^{X}(Y)$ is then the $L^{2}(\Omega)$ limit of $\delta^{X}\left(Y^{\pi}\right)$.

Proposition 4.4. Let $g:[0, T]^{2} \rightarrow \mathbb{R}^{e} \otimes \mathbb{R}^{d}$ be of the form $g(s, t)=$ $\mathbb{1}_{[0, t)}(s) \tilde{g}_{1}(t) \tilde{g}_{2}(s)$, where $\tilde{g}_{1} \in \mathcal{C}_{\mathrm{pw}}^{p \text {-var }}\left([0, T] ; \mathbb{R}^{e} \otimes \mathbb{R}^{e}\right)$ and $\tilde{g}_{2} \in \mathcal{C}_{\mathrm{pw}}^{p \text {-var }}([0, T]$; $\left.\mathbb{R}^{e} \otimes \mathbb{R}^{d}\right)$. Let $R$ be continuous and of finite $2 D \rho$-variation, $\rho \in\left[1, \frac{3}{2}\right)$, and we assume that $\frac{1}{p}+\frac{1}{\rho}>1$. For any partition $\pi=\left\{r_{i}\right\}$ of $[0, T]$, let $g^{\pi}:[0, T]^{2} \rightarrow$ $\mathbb{R}^{e} \otimes \mathbb{R}^{d}$ denote

$$
g^{\pi}(s, t):=\sum_{i} g\left(s, r_{i}\right) \mathbb{1}_{\left[r_{i}, r_{i+1}\right)}(t)
$$

Then

$$
\begin{aligned}
\int_{[0, T]^{2}} & \left(\int_{[0, T]^{2}}\left\langle\left(g^{\pi}-g\right)(u, s),\left(g^{\pi}-g\right)(v, t)\right\rangle_{\mathbb{R}^{e} \otimes \mathbb{R}^{d}} \mathrm{~d} R(u, v)\right) \mathrm{d} R(s, t) \\
\rightarrow & \rightarrow 0 .
\end{aligned}
$$

REMARK 4.5. Here and henceforth, we canonically identify 2-tensors with matrices, and $\tilde{g}_{1}(t) \tilde{g}_{2}(s)$ denotes matrix multiplication of $\tilde{g}_{1}(t)$ with $\tilde{g}_{2}(s)$.

PROOF. Similar to the proof of Lemma 4.1, we will first partition $[0, T]^{2} \times$ $[0, T]^{2}$ into subregions $A \times B=\left(\left[a_{1}, a_{2}\right] \times\left[a_{3}, a_{4}\right]\right) \times\left(\left[b_{1}, b_{2}\right] \times\left[b_{3}, b_{4}\right]\right)$ on which the integrand is continuous (shrinking each region if necessary to deal with discontinuities at the boundaries), then sum the results to obtain the full proof.

Under the conditions imposed on $g$, we will show that the 4D-integral in (76) can be written as an iterated 2D-integral and

$$
\begin{gathered}
\int_{A}\left(\int_{B}\left\langle\left(g-g^{\pi}\right)(u, s),\left(g-g^{\pi}\right)(v, t)\right\rangle_{\mathbb{R}^{e} \otimes \mathbb{R}^{d}} \mathrm{~d} R(u, v)\right) \mathrm{d} R(s, t) \\
=\sum_{i, j} \int_{\left[r_{i}, r_{i+1}\right] \times\left[r_{j}, r_{j+1}\right]} I^{i, j}(s, t) \mathrm{d} R(s, t) \stackrel{\|\pi\| \rightarrow 0}{\longrightarrow} 0,
\end{gathered}
$$


where

$$
I^{i, j}(s, t):=\int_{B}\left\langle g(u, s)-g\left(u, r_{i}\right), g(v, t)-g\left(v, r_{j}\right)\right\rangle_{\mathbb{R}^{e} \otimes \mathbb{R}^{d}} \mathrm{~d} R(u, v) .
$$

First, observe that for any $r \leq s$,

$$
\begin{aligned}
g(u, s)-g(u, r) & =\mathbb{1}_{[0, s)}(u) \tilde{g}_{1}(s) \tilde{g}_{2}(u)-\mathbb{1}_{[0, r)}(u) \tilde{g}_{1}(r) \tilde{g}_{2}(u) \\
& =\mathbb{1}_{[0, r)}(u)\left(\tilde{g}_{1}(s)-\tilde{g}_{1}(r)\right) \tilde{g}_{2}(u)+\mathbb{1}_{[r, s)}(u) \tilde{g}_{1}(s) \tilde{g}_{2}(u) .
\end{aligned}
$$

Thus for $(s, t) \in\left[r_{i}, r_{i+1}\right] \times\left[r_{j}, r_{j+1}\right] \subset A$,

$$
I^{i, j}(s, t)=I_{1}^{i, j}(s, t)+I_{2}^{i, j}(s, t)+I_{3}^{i, j}(s, t)+I_{4}^{i, j}(s, t),
$$

where

$$
\begin{aligned}
I_{1}^{i, j}(s, t):= & \sum_{l, m, n, k}\left(\tilde{g}_{1}(s)-\tilde{g}_{1}\left(r_{i}\right)\right)_{l m}\left(\tilde{g}_{1}(t)-\tilde{g}_{1}\left(r_{j}\right)\right)_{l n} \\
& \times \int_{\left[b_{1}, r_{i}\right] \times\left[b_{3}, r_{j}\right]}\left(\tilde{g}_{2}(u)\right)_{m k}\left(\tilde{g}_{2}(v)\right)_{n k} \mathrm{~d} R(u, v), \\
I_{2}^{i, j}(s, t):= & \sum_{l, m, n, k}\left(\tilde{g}_{1}(s)\right)_{l m}\left(\tilde{g}_{1}(t)-\tilde{g}_{1}\left(r_{j}\right)\right)_{l n} \\
& \times \int_{\left[r_{i}, s\right] \times\left[b_{3}, r_{j}\right]}\left(\tilde{g}_{2}(u)\right)_{m k}\left(\tilde{g}_{2}(v)\right)_{n k} \mathrm{~d} R(u, v), \\
I_{3}^{i, j}(s, t):= & \sum_{l, m, n, k}\left(\tilde{g}_{1}(s)-\tilde{g}_{1}\left(r_{i}\right)\right)_{l m}\left(\tilde{g}_{1}(t)\right)_{l n} \\
& \times \int_{\left[b_{1}, r_{i}\right] \times\left[r_{j}, t\right]}\left(\tilde{g}_{2}(u)\right)_{m k}\left(\tilde{g}_{2}(v)\right)_{n k} \mathrm{~d} R(u, v), \\
I_{4}^{i, j}(s, t):= & \sum_{l, m, n, k}\left(\tilde{g}_{1}(s)\right)_{l m}\left(\tilde{g}_{1}(t)\right)_{l n} \\
& \times \int_{\left[r_{i}, s\right] \times\left[r_{j}, t\right]}\left(\tilde{g}_{2}(u)\right)_{m k}\left(\tilde{g}_{2}(v)\right)_{n k} \mathrm{~d} R(u, v),
\end{aligned}
$$

and $(g)_{l m}$ denotes the $(l, m)$ th entry of the matrix $g$. Note also that it is possible that $r_{i} \leq b_{1}$ or $r_{j} \leq b_{3}$, in which case we define the integral to be zero.

For $k=1,2,3$, since the summands in $I_{k}^{i, j}(s, t)$ are products of $1 \mathrm{D}$ functions, we have the following bounds on the $2 \mathrm{D} p$ th-variation of $I_{k}^{i, j}(s, t)$ in $\left[r_{i}, r_{i+1}\right] \times$ 
$\left[r_{j}, r_{j+1}\right]:$

$$
\begin{aligned}
\left\|I_{1}^{i, j}\right\|_{p \text {-var; }\left[r_{i}, r_{i+1}\right] \times\left[r_{j}, r_{j+1}\right]} & \\
\leq & C_{p, \rho, d}\left\|\tilde{g}_{2}\right\|_{\mathcal{V} p ;[0, T]}^{2}\|R\|_{\rho \text {-var; }[0, T]^{2}} \| \\
& \times \tilde{g}_{1}\left\|_{p \text {-var; }\left[r_{i}, r_{i+1}\right]}\right\| \tilde{g}_{1} \|_{p \text {-var; }\left[r_{j}, r_{j+1}\right]}, \\
\left\|I_{2}^{i, j}\right\|_{p \text {-var; }\left[r_{i}, r_{i+1}\right] \times\left[r_{j}, r_{j+1}\right]} & \leq C_{d}\left\|\tilde{g}_{1}\right\|_{p \text {-var; }\left[r_{j}, r_{j+1}\right]} \\
& \times\left(\left\|\tilde{g}_{1}\right\|_{p \text {-var; }\left[r_{i}, r_{i+1}\right]}\left\|h_{1}\right\|_{\infty}+\left\|\tilde{g}_{1}\right\|_{\infty}\left\|h_{1}\right\|_{p \text {-var; }\left[r_{i}, r_{i+1}\right]}\right), \\
\left\|I_{3}^{i, j}\right\|_{p \text {-var; }\left[r_{i}, r_{i+1}\right] \times\left[r_{j}, r_{j+1}\right]} & \\
\leq & C_{d}\left\|\tilde{g}_{1}\right\|_{p \text {-var; }\left[r_{i}, r_{i+1}\right]} \\
& \times\left(\left\|\tilde{g}_{1}\right\|_{p \text {-var; }\left[r_{j}, r_{j+1}\right]}\left\|h_{2}\right\|_{\infty}+\left\|\tilde{g}_{1}\right\|_{\infty}\left\|h_{2}\right\|_{p \text {-var; }\left[r_{j}, r_{j+1}\right]}\right) .
\end{aligned}
$$

Here, $h_{1}$ and $h_{2}$ denote the functions (suppressing the dependence on $m, n$ and $k$ in the notation since the bounds are independent of them)

$$
\begin{aligned}
h_{1}(s) & :=\int_{\left[r_{i}, s\right] \times\left[b_{3}, r_{j}\right]}\left(\tilde{g}_{2}(u)\right)_{m k}\left(\tilde{g}_{2}(v)\right)_{n k} \mathrm{~d} R(u, v), \\
h_{2}(t) & :=\int_{\left[b_{1}, r_{i}\right] \times\left[r_{j}, t\right]}\left(\tilde{g}_{2}(u)\right)_{m k}\left(\tilde{g}_{2}(v)\right)_{n k} \mathrm{~d} R(u, v) .
\end{aligned}
$$

Choosing $\varepsilon$ sufficiently small such that $\rho+\varepsilon<p$ and $\frac{1}{p}+\frac{1}{\rho+\varepsilon}>1$, we have

$$
\begin{aligned}
\left\|h_{1}\right\|_{p \text {-var; }\left[r_{i}, r_{i+1}\right]} & \leq\left\|h_{1}\right\|_{\rho+\varepsilon \text {-var; }\left[r_{i}, r_{i+1}\right]} \\
& \leq C_{p, \rho}\left\|\tilde{g}_{2}\right\|_{\mathcal{V}^{p} ;[0, T]}^{2} \omega_{R, \rho+\varepsilon}\left(\left[r_{i}, r_{i+1}\right] \times[0, T]\right)^{\frac{1}{\rho+\varepsilon}},
\end{aligned}
$$

(78) $\left\|h_{2}\right\|_{p \text {-var; }\left[r_{j}, r_{j+1}\right]} \leq\left\|h_{2}\right\|_{\rho+\varepsilon \text {-var; }\left[r_{j}, r_{j+1}\right]}$

$$
\begin{aligned}
& \leq C_{p, \rho}\left\|\tilde{g}_{2}\right\|_{\mathcal{V} p^{p}[0, T]}^{2} \omega_{R, \rho+\varepsilon}\left([0, T] \times\left[r_{j}, r_{j+1}\right]\right)^{\frac{1}{\rho+\varepsilon}}, \\
\left\|h_{1}\right\|_{\infty},\left\|h_{2}\right\|_{\infty} & \leq C_{p, \rho}\left\|\tilde{g}_{2}\right\|_{\mathcal{V}^{p} ;[0, T]}^{2}\|R\|_{\rho-\operatorname{var} ;[0, T]^{2}}
\end{aligned}
$$

From (77) and (78), we see that the 2D pth-variations of $I_{1}^{i, j}, I_{2}^{i, j}$ and $I_{3}^{i, j}$ over $[s, t] \times[u, v] \subset\left[a_{1}, a_{2}\right] \times\left[a_{3}, a_{4}\right]$ are controlled respectively by

$$
\begin{aligned}
& \omega_{I_{1}}([s, t] \times[u, v]) \\
& \quad:=C_{1}\left\|\tilde{g}_{2}\right\|_{\mathcal{V}^{p} ;[0, T]}^{2 p}\left\|\tilde{g}_{1}\right\|_{p \text {-var; }[s, t]}^{p}\left\|\tilde{g}_{1}\right\|_{p \text {-var; }[u, v]}^{p}\|R\|_{\rho \text {-var; }[0, T]^{2}}^{p}, \\
& \omega_{I_{2}}([s, t] \times[u, v])
\end{aligned}
$$




$$
\begin{aligned}
:= & C_{2}\left\|\tilde{g}_{2}\right\|_{\mathcal{V}^{p} ;[0, T]}^{2 p}\left\|\tilde{g}_{1}\right\|_{p-\operatorname{var} ;[u, v]}^{p} \\
& \times\left(\left\|\tilde{g}_{1}\right\|_{p^{-\operatorname{var} ;[s, t]}}^{p}\|R\|_{\rho \text {-var; }[0, T]^{2}}^{p}+\left\|\tilde{g}_{1}\right\|_{\infty}^{p} \omega_{R, \rho+\varepsilon}([s, t] \times[0, T])^{\frac{p}{\rho+\varepsilon}}\right), \\
\omega_{I_{3}}([s, t] \times[u, v]) & \\
:= & C_{3}\left\|\tilde{g}_{2}\right\|_{\mathcal{V}^{p} ;[0, T]}^{2 p}\left\|\tilde{g}_{1}\right\|_{p-\operatorname{var} ;[s, t]}^{p} \\
& \times\left(\left\|\tilde{g}_{1}\right\|_{p^{-\operatorname{var} ;[u, v]}}^{p}\|R\|_{\rho-\operatorname{var} ;[0, T]^{2}}^{p}+\left\|\tilde{g}_{1}\right\|_{\infty}^{p} \omega_{R, \rho+\varepsilon}([0, T] \times[u, v])^{\frac{p}{\rho+\varepsilon}}\right) .
\end{aligned}
$$

For $I_{4}^{i, j}(s, t)$, if we let

$$
h_{3}(s, t):=\int_{\left[r_{i}, s\right] \times\left[r_{j}, t\right]}\left(\tilde{g}_{2}(u)\right)_{m k}\left(\tilde{g}_{2}(v)\right)_{n k} \mathrm{~d} R(u, v),
$$

we have

$$
\begin{aligned}
\left\|h_{3}\right\|_{p-\operatorname{var} ;\left[r_{i}, r_{i+1}\right] \times\left[r_{j}, r_{j+1}\right]} & \leq\left\|h_{3}\right\|_{\rho+\varepsilon-\operatorname{var} ;\left[r_{i}, r_{i+1}\right] \times\left[r_{j}, r_{j+1}\right]} \\
& \leq C_{p, \rho}\left\|\tilde{g}_{2}\right\|_{\mathcal{V} p ;[0, T]}^{2} \omega_{R, \rho+\varepsilon}\left(\left[r_{i}, r_{i+1}\right] \times\left[r_{j}, r_{j+1}\right]\right)^{\frac{1}{\rho+\varepsilon}} .
\end{aligned}
$$

From Lemma 4.6, we then conclude that the 2D $p$ th-variation of $I_{4}^{i, j}$ over $[s, t] \times$ $[u, v]$ is controlled by

$$
\begin{aligned}
\omega_{I_{4}}([s, t] \times[u, v]):= & C\left\|\tilde{g}_{2}\right\|_{\mathcal{V} p ;[0, T]}^{2 p} \omega_{R, \rho+\varepsilon}([s, t] \times[u, v])^{\frac{p}{\rho+\varepsilon}} \\
& \times\left(\left\|\tilde{g}_{1}\right\|_{\infty}^{p}+\left\|\tilde{g}_{1}\right\|_{p \text {-var; }[s, t]}^{p}\right)\left(\left\|\tilde{g}_{1}\right\|_{\infty}^{p}+\left\|\tilde{g}_{1}\right\|_{p \text {-var; }[u, v]}^{p}\right) .
\end{aligned}
$$

Now define $\omega$ as

$$
\omega([s, t] \times[u, v])=\omega_{I}^{\frac{1}{\theta p}}([s, t] \times[u, v]) \omega_{R, \rho+\varepsilon}^{\frac{1}{\theta(\rho+\varepsilon)}}([s, t] \times[u, v]),
$$

where $\omega_{I}$ denotes the control $\omega_{I_{1}}+\omega_{I_{2}}+\omega_{I_{3}}+\omega_{I_{4}}$ and $\theta=\frac{1}{p}+\frac{1}{\rho+\varepsilon}$. Then observing that $I^{i, j}\left(r_{i}, \cdot\right)=I^{i, j}\left(\cdot, r_{j}\right)=0$ for all $i, j$, we use Young's inequality (24) to obtain

$$
\begin{aligned}
& \sum_{i, j} \int_{\left[r_{i}, r_{i+1}\right] \times\left[r_{j}, r_{j+1}\right]} I^{i, j}(s, t) \mathrm{d} R(s, t) \\
& \quad \leq \sum_{i, j} \omega^{\theta}\left(\left[r_{i}, r_{i+1}\right] \times\left[r_{j}, r_{j+1}\right]\right) \rightarrow 0 .
\end{aligned}
$$

The following lemma was used in Proposition 4.4.

LEMMA 4.6. Let $g_{1} \in \mathcal{C}^{p \text {-var }}\left(\left[s_{1}, s_{2}\right] ; \mathbb{R}\right)$ and $g_{2} \in \mathcal{C}^{p \text {-var }}\left(\left[t_{1}, t_{2}\right] ; \mathbb{R}\right)$. Given a $2 D$ control $\omega$, let $f \in \mathcal{C}^{p \text {-var }}\left(\left[s_{1}, s_{2}\right] \times\left[t_{1}, t_{2}\right] ; \mathbb{R}\right)$ have finite $2 D$ p-variation controlled by $\omega$. In addition, assume that $f\left(s_{1}, t\right)=f\left(s, t_{1}\right)=0$ for all $s, t$ in 
$\left[s_{1}, s_{2}\right] \times\left[t_{1}, t_{2}\right]$. Then the $2 D$ pth-variation of $f(u, v) g_{1}(u) g_{2}(v)$ over $\left[s_{1}, s_{2}\right] \times$ $\left[t_{1}, t_{2}\right]$ is controlled by

$$
4^{p-1} \omega\left(\left[s_{1}, s_{2}\right] \times\left[t_{1}, t_{2}\right]\right)\left(\left\|g_{1}\right\|_{\infty}^{p}+\left\|g_{1}\right\|_{p-\operatorname{var} ;\left[s_{1}, s_{2}\right]}^{p}\right)\left(\left\|g_{2}\right\|_{\infty}^{p}+\left\|g_{2}\right\|_{p \text {-var; }\left[t_{1}, t_{2}\right]}^{p}\right) .
$$

PROOF. Let $\left\{\left(u_{i}, v_{j}\right)\right\}$ be any partition of $\left[s_{1}, s_{2}\right] \times\left[t_{1}, t_{2}\right]$. We have

$$
\begin{aligned}
\sum_{i, j} \mid f & \left.g_{1}\left(\begin{array}{cc}
u_{i} & u_{i+1} \\
v_{j} & v_{j+1}
\end{array}\right)\right|^{p} \\
= & \sum_{i, j} \mid\left(f\left(u_{i}, v_{j}\right)-f\left(u_{i}, v_{j+1}\right)\right) g_{1}\left(u_{i}\right) \\
& +\left.\left(f\left(u_{i+1}, v_{j+1}\right)-f\left(u_{i+1}, v_{j}\right)\right) g_{1}\left(u_{i+1}\right)\right|^{p} \\
\leq & 2^{p-1}\left[\sum_{i, j}\left|f\left(\begin{array}{ll}
u_{i} & u_{i+1} \\
v_{j} & v_{j+1}
\end{array}\right) g_{1}\left(u_{i}\right)\right|^{p}\right. \\
& \left.+\sum_{i}\left|g_{1}\left(u_{i+1}\right)-g_{1}\left(u_{i}\right)\right|^{p} \sum_{j}\left|f\left(\begin{array}{ll}
s_{1} & u_{i+1} \\
v_{j} & v_{j+1}
\end{array}\right)\right|^{p}\right] \\
\leq & 2^{p-1}\left[\left\|g_{1}\right\|_{\infty}^{p}\|f\|_{p-\mathrm{var}}^{p}+\|f\|_{p \text {-var }}^{p} \sum_{i}\left|g_{1}\left(u_{i+1}\right)-g_{1}\left(u_{i}\right)\right|^{p}\right],
\end{aligned}
$$

which tells us that the 2D pth-variation of $f g_{1}$ is controlled by

$$
2^{p-1} \omega\left(\left[s_{1}, s_{2}\right] \times\left[t_{1}, t_{2}\right]\right)\left(\left\|g_{1}\right\|_{\infty}^{p}+\left\|g_{1}\right\|_{p-\operatorname{var}\left[\left[s_{1}, s_{2}\right]\right.}^{p}\right) .
$$

Repeating the same procedure with $f g_{1}$ [controlled by (80)] in place of $f$ and $g_{2}$ in place of $g_{1}$ completes the proof.

PROPOSITION 4.7. Let $g(s, t)=\mathbb{1}_{[0, t)}(s) \tilde{g}_{1}(t) \tilde{g}_{2}(s)$, where $\tilde{g}_{1}, \tilde{g}_{2} \in$ $\mathcal{C}_{\mathrm{pw}}^{p \text {-var }}\left([0, T] ; \mathbb{R}^{d} \otimes \mathbb{R}^{d}\right)$, and let $g^{\pi}$ be defined as in $(75)$. Let $R$ be of finite $2 D$ $\rho$-variation, $\rho \in\left[1, \frac{3}{2}\right)$, and we assume that $\frac{1}{p}+\frac{1}{\rho}>1$. Then $g \in \mathcal{H}_{1}^{d} \otimes \mathcal{H}_{1}^{d}$, with norm given by

$$
\|g\|_{\mathcal{H}_{1}^{d} \otimes \mathcal{H}_{1}^{d}}=\sqrt{\int_{[0, T]^{2}}\left(\int_{[0, T]^{2}}\langle g(u, s), g(v, t)\rangle_{\mathbb{R}^{d} \otimes \mathbb{R}^{d}} \mathrm{~d} R(u, v)\right) \mathrm{d} R(s, t),}
$$

and

$$
\left\|g^{\pi}-g\right\|_{\mathcal{H}_{1}^{d} \otimes \mathcal{H}_{1}^{d}} \rightarrow 0
$$

as $\|\pi\| \rightarrow 0$. 
Proof. Given a $d$-by- $d$ matrix function $A(s)$, let $a_{j}^{(i)}(s)$ denote the $(i, j)$ th entry of $A(s)$. Using the canonical identification,

$$
A(s) \mathbb{1}_{[a, b)}(t) \simeq \sum_{j=1}^{d} \sum_{k=1}^{d} a_{j}^{(k)}(s) e_{k} \otimes \mathbb{1}_{[a, b)}^{(j)}(t), \quad a, b \in[0, T],
$$

we see that $g^{\pi}$ is a member of $\mathcal{W}_{\rho}^{d} \otimes \mathcal{H}_{1}^{d}$, and thus lies in $\mathcal{H}_{1}^{d} \otimes \mathcal{H}_{1}^{d}$ by Proposition 4.2. Furthermore, we can compute the square of its norm

$$
\begin{aligned}
\left\|g^{\pi}\right\|_{\mathcal{H}_{1}^{d} \otimes \mathcal{H}_{1}^{d}}^{2} & =\sum_{k, l} \int_{[0, T]^{2}} \sum_{j=1}^{d}\left\langle g_{j}\left(u, r_{k}\right), g_{j}\left(v, r_{l}\right)\right\rangle_{\mathbb{R}^{d}} \mathrm{~d} R(u, v) R\left(\begin{array}{cc}
r_{k} & r_{k+1} \\
r_{l} & r_{l+1}
\end{array}\right) \\
& =\int_{[0, T]^{2}}\left(\int_{[0, T]^{2}}\left\langle g^{\pi}(u, s), g^{\pi}(v, t)\right\rangle_{\mathbb{R}^{d} \otimes \mathbb{R}^{d}} \mathrm{~d} R(u, v)\right) \mathrm{d} R(s, t) .
\end{aligned}
$$

Taking any sequence of partitions $\pi(n)$ with vanishing mesh, we know that $g^{\pi(n)}$ is Cauchy as $n \rightarrow \infty$ by Proposition 4.4 , and we identify $g$ with its limit in $\mathcal{H}_{1}^{d} \otimes \mathcal{H}_{1}^{d}$. Invoking Proposition 4.4 again gives us (81) and (82).

\subsection{The Itô-Skorohod isometry revisited.}

THEOREM 4.8. Let $X$ be a continuous, centered Gaussian process in $\mathbb{R}^{d}$ with i.i.d. components, and assume that its continuous covariance function satisfies $\|R\|_{\rho \text {-var; }[0, T]^{2}}<\infty$ for some $\rho \in\left[1, \frac{3}{2}\right)$. Given $p$ satisfying $\frac{1}{p}+\frac{1}{\rho}>1$, let $Y$ be a random variable which satisfies, almost surely:

(i) $Y \in \mathcal{C}_{\mathrm{pw}}^{p \text {-var }}\left([0, T] ; \mathbb{R}^{d}\right)$,

(ii) $\mathcal{D} Y:[0, T]^{2} \rightarrow \mathbb{R}^{d} \otimes \mathbb{R}^{d}$ is of the form $\mathbb{1}_{[0, t)}(s) \tilde{g}_{1}(t) \tilde{g}_{2}(s)$, where $\tilde{g}_{1}, \tilde{g}_{2}$ are both in $\mathcal{C}_{\mathrm{pw}}^{p \text {-var }}\left([0, T] ; \mathbb{R}^{d} \otimes \mathbb{R}^{d}\right)$.

Then $\lim _{\|\pi\| \rightarrow 0} Y^{\pi}=Y$ in $\mathbb{D}^{1,2}\left(\mathcal{H}_{1}^{d}\right)$ if and only if

$$
\mathbb{E}\left[\int_{[0, T]^{2}}\left\langle Y_{s}^{\pi}-Y_{s}, Y_{t}^{\pi}-Y_{t}\right\rangle_{\mathbb{R}^{d}} \mathrm{~d} R(s, t)\right] \rightarrow 0
$$

and

$$
\mathbb{E}\left[\int_{[0, T]^{2}}\left(\int_{[0, T]^{2}}\left\langle\mathcal{D}_{r}\left(Y_{t}^{\pi}-Y_{t}\right), \mathcal{D}_{q}\left(Y_{s}^{\pi}-Y_{s}\right)\right\rangle_{\mathbb{R}^{d} \otimes \mathbb{R}^{d}} \mathrm{~d} R(r, q)\right) \mathrm{d} R(s, t)\right] \rightarrow 0
$$

as $\|\pi\| \rightarrow 0$, in which case $\lim _{\|\pi\| \rightarrow 0} \mathbb{E}\left[\delta^{X}\left(Y^{\pi}-Y\right)^{2}\right]=0$ and $\mathbb{E}\left[\delta^{X}(Y)^{2}\right]$ is equal to

$$
\mathbb{E}\left[\int_{[0, T]^{2}}\left\langle Y_{s}, Y_{t}\right\rangle_{\mathbb{R}^{d}} \mathrm{~d} R(s, t)\right]+\mathbb{E}\left[\int_{[0, T]^{4}} \operatorname{tr}\left(\mathcal{D}_{r} Y_{t} \mathcal{D}_{q} Y_{S}\right) \mathrm{d} R(s, r) \mathrm{d} R(t, q)\right] .
$$


PROOF. For a Malliavin-smooth real-valued random variable $F$, we will write

$$
\mathcal{D} F=\left(\mathcal{D}^{(1)} F, \ldots, \mathcal{D}^{(d)} F\right) \in \mathcal{H}_{1}^{d},
$$

which means that $\mathcal{D}_{s}^{(j)} Y_{t}^{(i)}$ will denote the $(i, j)$ th-entry of the matrix $\mathcal{D}_{s} Y_{t}$.

From Propositions 4.2 and 4.7,

$$
\mathbb{E}\left[\left\|Y^{\pi}-Y\right\|_{\mathcal{H}_{1}^{d}}^{2}\right]=\mathbb{E}\left[\int_{[0, T]^{2}}\left\langle Y_{s}^{\pi}-Y_{s}, Y_{t}^{\pi}-Y_{t}\right\rangle_{\mathbb{R}^{d}} \mathrm{~d} R(s, t)\right],
$$

and $\mathbb{E}\left[\left\|\mathcal{D} Y^{\pi}-\mathcal{D} Y\right\|_{\mathcal{H}_{1}^{d} \otimes \mathcal{H}_{1}^{d}}^{2}\right]$ is equal to

$$
\mathbb{E}\left[\int_{[0, T]^{2}}\left(\int_{[0, T]^{2}}\left\langle\mathcal{D}_{r}\left(Y_{t}^{\pi}-Y_{t}\right), \mathcal{D}_{q}\left(Y_{s}^{\pi}-Y_{s}\right)\right\rangle_{\mathbb{R}^{d} \otimes \mathbb{R}^{d}} \mathrm{~d} R(r, q)\right) \mathrm{d} R(s, t)\right] .
$$

Furthermore, Itô-Skorohod isometry (see [29]) gives us

$$
\begin{aligned}
\mathbb{E}\left[\delta^{X}(Y)^{2}\right] & =\mathbb{E}\left[\|Y\|_{\mathcal{H}_{1}^{d}}^{2}\right]+\mathbb{E}[\operatorname{trace}(\mathcal{D} Y \circ \mathcal{D} Y)] \\
& =\lim _{\|\pi\| \rightarrow 0} \mathbb{E}\left[\left\|Y^{\pi}\right\|_{\mathcal{H}_{1}^{d}}^{2}\right]+\lim _{\|\pi\| \rightarrow 0} \mathbb{E}\left[\operatorname{trace}\left(\mathcal{D} Y^{\pi} \circ \mathcal{D} Y^{\pi}\right)\right],
\end{aligned}
$$

since

$$
\mathbb{E}\left[\delta^{X}\left(Y^{\pi}-Y\right)^{2}\right] \leq \mathbb{E}\left[\left\|Y^{\pi}-Y\right\|_{\mathcal{H}_{1}^{d}}^{2}\right]+\mathbb{E}\left[\left\|\mathcal{D} Y^{\pi}-\mathcal{D} Y\right\|_{\mathcal{H}_{1}^{d} \otimes \mathcal{H}_{1}^{d}}^{2}\right] .
$$

Recall that the trace term is given by

$$
\operatorname{trace}(\mathcal{D} Y \circ \mathcal{D} Y)=\sum_{m=1}^{\infty}\left\langle\mathcal{D} Y\left(h_{m}\right),(\mathcal{D} Y)^{*}\left(h_{m}\right)\right\rangle_{\mathcal{H}_{1}^{d}},
$$

where $\left\{h_{m}\right\}$ denotes any orthonormal basis for $\mathcal{H}_{1}^{d}$ and

$$
\begin{aligned}
\mathcal{D} Y\left(h_{m}\right)(r) & =\sum_{k=1}^{d}\left[\left\langle\mathcal{D} . Y_{r}^{(k)}, h_{m}(\cdot)\right\rangle_{\mathcal{H}_{1}^{d}}\right] e_{k}, \\
(\mathcal{D} Y)^{*}\left(h_{m}\right)(r) & =\sum_{k=1}^{d}\left[\left\langle\mathcal{D}_{r}^{(k)} Y ., h_{m}(\cdot)\right\rangle_{\mathcal{H}_{1}^{d}}\right] e_{k}, \quad r \in[0, T], m=1, \ldots
\end{aligned}
$$

For the first term, we have

$$
\begin{aligned}
\lim _{\|\pi\| \rightarrow 0} \mathbb{E}\left[\left\|Y^{\pi}\right\|_{\mathcal{H}_{1}^{d}}^{2}\right] & =\lim _{\|\pi\| \rightarrow 0} \mathbb{E}\left[\sum_{j, k}\left\langle Y_{t_{j}}, Y_{t_{k}}\right\rangle_{\mathbb{R}^{d}} R\left(\begin{array}{cc}
t_{j} & t_{j+1} \\
t_{k} & t_{k+1}
\end{array}\right)\right] \\
& =\mathbb{E}\left[\int_{[0, T]^{2}}\left\langle Y_{s}, Y_{t}\right\rangle_{\mathbb{R}^{d}} \mathrm{~d} R(s, t)\right],
\end{aligned}
$$

and for the second term, we need to compute

$$
\mathbb{E}\left[\operatorname{trace}\left(\mathcal{D} Y^{\pi} \circ \mathcal{D} Y^{\pi}\right)\right]=\mathbb{E}\left[\sum_{m=1}^{\infty}\left\langle\mathcal{D} Y^{\pi}\left(h_{m}\right),\left(\mathcal{D} Y^{\pi}\right)^{*}\left(h_{m}\right)\right\rangle_{\mathcal{H}_{1}^{d}}\right] .
$$


We have

$$
\begin{aligned}
& \mathcal{D} Y^{\pi}\left(h_{m}\right)(r)=\sum_{k=1}^{d}\left[\sum_{i}\left\langle\mathcal{D} . Y_{t_{i}}^{(k)}, h_{m}(\cdot)\right\rangle_{\mathcal{H}_{1}^{d}} \mathbb{1}_{\Delta_{i}}(r)\right] e_{k} \quad \text { and } \\
& \left(\mathcal{D} Y^{\pi}\right)^{*}\left(h_{m}\right)(r)=\sum_{k=1}^{d}\left[\sum_{j}\left\langle\mathcal{D}_{r}^{(k)} Y_{t_{j}} \mathbb{1}_{\Delta_{j}}(\cdot), h_{m}(\cdot)\right\rangle_{\mathcal{H}_{1}^{d}}\right] e_{k}, \\
& r \in[0, T], m=1, \ldots,
\end{aligned}
$$

which yields

$$
\begin{aligned}
& \mathbb{E}\left[\sum_{m=1}^{\infty}\left\langle\mathcal{D} Y^{\pi} h_{m},\left(\mathcal{D} Y^{\pi}\right)^{*} h_{m}\right\rangle_{\mathcal{H}_{1}^{d}}\right] \\
& =\mathbb{E}\left[\sum_{i, j} \sum_{k=1}^{d} \sum_{m=1}^{\infty}\left\langle\left\langle\mathcal{D}_{r}^{(k)} Y_{t_{j}} \mathbb{1}_{\Delta_{j}}(\cdot), h_{m}(\cdot)\right\rangle_{\mathcal{H}_{1}^{d}},\left\langle\mathcal{D} \cdot Y_{t_{i}}^{(k)}, h_{m}(\cdot)\right\rangle_{\mathcal{H}_{1}^{d}} \mathbb{1}_{\Delta_{i}}(r)\right\rangle_{\mathcal{H}_{1}}\right] \\
& =\mathbb{E}\left[\sum_{i, j} \sum_{k=1}^{d}\left\langle\sum_{m=1}^{\infty}\left\langle\mathcal{D}_{r}^{(k)} Y_{t_{j}} \mathbb{1}_{\Delta_{j}}(\cdot), h_{m}(\cdot)\right\rangle_{\mathcal{H}_{1}^{d}}\left\langle\mathcal{D} \cdot Y_{t_{i}}^{(k)}, h_{m}(\cdot)\right\rangle_{\mathcal{H}_{1}^{d}}, \mathbb{1}_{\Delta_{i}}(r)\right\rangle_{\mathcal{H}_{1}}\right] \\
& =\mathbb{E}\left[\sum_{i, j} \sum_{k=1}^{d}\left\langle\left\langle\mathcal{D}_{r}^{(k)} Y_{t_{j}} \mathbb{1}_{\Delta_{j}}(\cdot), \mathcal{D} \cdot Y_{t_{i}}^{(k)}\right\rangle_{\mathcal{H}_{1}^{d}}, \mathbb{1}_{\Delta_{i}}(r)\right\rangle_{\mathcal{H}_{1}}\right],
\end{aligned}
$$

where $r$ is the variable for the outer $\mathcal{H}_{1}$-inner product.

Since

$$
\begin{aligned}
\left\langle\mathcal{D}_{r}^{(k)} Y_{t_{j}} \mathbb{1}_{\Delta_{j}}(\cdot), \mathcal{D} . Y_{t_{i}}^{(k)}\right\rangle_{\mathcal{H}_{1}^{d}} & =\sum_{l=1}^{d}\left\langle\mathcal{D}_{r}^{(k)} Y_{t_{j}}^{(l)} \mathbb{1}_{\Delta_{j}}(\cdot), \mathcal{D}^{(l)} Y_{t_{i}}^{(k)}\right\rangle_{\mathcal{H}_{1}} \\
& =\sum_{l=1}^{d} \mathcal{D}_{r}^{(k)} Y_{t_{j}}^{(l)}\left\langle\mathbb{1}_{\Delta_{j}}(\cdot), \mathcal{D}^{(l)} Y_{t_{i}}^{(k)}\right\rangle_{\mathcal{H}_{1}},
\end{aligned}
$$

with $R\left(\Delta_{i}, \mathrm{~d} r\right)$ denoting $R\left(t_{i+1}, \mathrm{~d} r\right)-R\left(t_{i}, \mathrm{~d} r\right)$ [cf. (21)], we obtain $\mathbb{E}\left[\operatorname{trace}\left(\mathcal{D} Y^{\pi} \circ \mathcal{D} Y^{\pi}\right)\right]$

$$
\begin{aligned}
& =\mathbb{E}\left[\sum_{i, j} \sum_{k, l=1}^{d}\left\langle\mathcal{D}^{(k)} Y_{t_{j}}^{(l)}, \mathbb{1}_{\Delta_{i}}(\cdot)\right\rangle_{\mathcal{H}_{1}}\left\langle\mathcal{D}^{(l)} Y_{t_{i}}^{(k)}, \mathbb{1}_{\Delta_{j}}(\cdot)\right\rangle_{\mathcal{H}_{1}}\right] \\
& =\mathbb{E}\left[\sum_{i, j} \sum_{k, l=1}^{d} \int_{0}^{T} \mathcal{D}_{r}^{(k)} Y_{t_{j}}^{(l)} R\left(\Delta_{i}, \mathrm{~d} r\right) \int_{0}^{T} \mathcal{D}_{q}^{(l)} Y_{t_{i}}^{(k)} R\left(\Delta_{j}, \mathrm{~d} q\right)\right] \\
& \rightarrow \mathbb{E}\left[\int_{[0, T]^{4}} \operatorname{tr}\left(\mathcal{D}_{r} Y_{t} \mathcal{D}_{q} Y_{s}\right) \mathrm{d} R(s, r) \mathrm{d} R(t, q)\right] \text { as }\|\pi\| \rightarrow 0 .
\end{aligned}
$$


REMARK 4.9. In the case of standard Brownian motion, if we use the fact that $\mathrm{d} R(s, t)=\delta_{\{s=t\}} \mathrm{d} s \mathrm{~d} t$ in Theorem 4.8, we recover the usual Itô-Skorohod isometry

$$
\mathbb{E}\left[\delta^{X}(Y)^{2}\right]=\mathbb{E}\left[\int_{0}^{T}\left|Y_{t}\right|^{2} \mathrm{~d} t\right]+\mathbb{E}\left[\int_{[0, T]^{2}} \operatorname{tr}\left(\mathcal{D}_{t} Y_{s} \mathcal{D}_{s} Y_{t}\right) \mathrm{d} s \mathrm{~d} t\right] .
$$

\subsection{Riemann sum approximation to the Skorohod integral.}

Proposition 4.10. Let $X$ be a continuous, centered Gaussian process in $\mathbb{R}^{d}$ with i.i.d. components, and assume that its continuous covariance function satisfies $\|R\|_{\rho \text {-var; }[0, T]^{2}}<\infty$ for some $\rho \in\left[1, \frac{3}{2}\right)$. For $p \in[1,3)$, let $\mathbf{X} \in$ $\mathcal{C}^{0, p-\operatorname{var}}\left([0, T] ; G^{\lfloor p\rfloor}\left(\mathbb{R}^{d}\right)\right)$ denote the geometric rough path constructed from the limit of the piecewise-linear approximations of $X$.

Furthermore, let $Y \in \mathcal{C}^{p-\operatorname{var}}\left([0, T] ; \mathbb{R}^{d}\right)$ denote the path-level solution to

$$
\mathrm{d} Y_{t}=V\left(Y_{t}\right) \circ \mathrm{d} \mathbf{X}_{t}, \quad Y_{0}=y_{0},
$$

where $V \in \mathcal{C}_{b}^{\lfloor p\rfloor+1}\left(\mathbb{R}^{d} ; \mathbb{R}^{d} \otimes \mathbb{R}^{d}\right)$. Then $Y \in \mathbb{D}^{1,2}\left(\mathcal{H}_{1}^{d}\right)$ and

$$
\begin{aligned}
& \int_{0}^{T} Y_{r} \mathrm{~d} X_{r} \\
& \quad=\lim _{\left\|\pi=\left\{r_{i}\right\}\right\| \rightarrow 0} \sum_{i}\left[Y_{r_{i}}\left(X_{r_{i}, r_{i+1}}\right)-\int_{0}^{r_{i}} \operatorname{tr}\left[J_{r_{i} \leftarrow s}^{\mathbf{X}} V\left(Y_{S}\right)\right] R\left(\Delta_{i}, \mathrm{~d} s\right)\right],
\end{aligned}
$$

where the limit is taken in $L^{2}(\Omega)$.

PROOF. Using integration-by-parts, we have

$$
\begin{aligned}
\delta^{X}\left(Y^{\pi}\right) & =\sum_{i}\left[\left\langle Y_{r_{i}}, X_{r_{i}, r_{i+1}}\right\rangle_{\mathbb{R}^{d}}-\sum_{k=1}^{d}\left\langle\mathcal{D} \cdot Y_{r_{i}}^{(k)}, \mathbb{1}_{\left[r_{i}, r_{i+1}\right)}^{(k)}(\cdot)\right\rangle_{\mathcal{H}_{1}^{d}}\right] \\
& =\sum_{i}\left[\left\langle Y_{r_{i}}, X_{r_{i}, r_{i+1}}\right\rangle_{\mathbb{R}^{d}}-\int_{0}^{r_{i}} \operatorname{tr}\left[J_{r_{i} \leftarrow s}^{\mathbf{X}} V\left(Y_{s}\right)\right] R\left(\Delta_{i}, \mathrm{~d} s\right)\right] .
\end{aligned}
$$

From Propositions 4.2 and 4.7, we have $Y \in \mathcal{H}_{1}^{d}$ and $\mathcal{D} Y=\mathbb{1}_{[0, t)}(s) J_{t \leftarrow s}^{\mathbf{X}} V\left(Y_{s}\right) \in$ $\mathcal{H}_{1}^{d} \otimes \mathcal{H}_{1}^{d}$ almost surely. So in light of Theorem 4.8, we need to show that

$$
\mathbb{E}\left[\int_{[0, T]^{2}}\left\langle Y_{s}^{\pi}-Y_{s}, Y_{t}^{\pi}-Y_{t}\right\rangle_{\mathbb{R}^{d}} \mathrm{~d} R(s, t)\right] \rightarrow 0,
$$

and

$$
\begin{aligned}
& \mathbb{E}\left[\int_{[0, T]^{2}}\left(\int_{[0, T]^{2}}\left\langle\mathcal{D}_{u}\left(Y_{s}^{\pi}-Y_{s}\right), \mathcal{D}_{v}\left(Y_{t}^{\pi}-Y_{t}\right)\right\rangle_{\mathbb{R}^{d} \otimes \mathbb{R}^{d}} \mathrm{~d} R(u, v)\right) \mathrm{d} R(s, t)\right] \\
& \quad \rightarrow 0
\end{aligned}
$$

as $\|\pi\| \rightarrow 0$. 
For (86), we have

$$
\begin{aligned}
& \mathbb{E}\left[\int_{[0, T]^{2}}\left\langle Y_{s}^{\pi}-Y_{s}, Y_{t}^{\pi}-Y_{t}\right\rangle_{\mathbb{R}^{d}} \mathrm{~d} R(s, t)\right] \\
& \quad=\mathbb{E}\left[\sum_{i, j} \int_{r_{i}}^{r_{i+1}} \int_{r_{j}}^{r_{j+1}}\left\langle Y_{s}-Y_{r_{i}}, Y_{t}-Y_{r_{j}}\right\rangle_{\mathbb{R}^{d}} \mathrm{~d} R(s, t)\right],
\end{aligned}
$$

and from Lemma 4.1 with $\omega$ and $\theta$ defined as in (70),

$$
\begin{gathered}
\sum_{i, j}\left|\int_{r_{i}}^{r_{i+1}} \int_{r_{j}}^{r_{j+1}}\left\langle Y_{s}-Y_{r_{i}}, Y_{t}-Y_{r_{j}}\right\rangle_{\mathbb{R}^{d}} \mathrm{~d} R(s, t)\right| \\
\leq C_{p, \rho} \sum_{i, j} \omega^{\theta}\left(\left[r_{i}, r_{i+1}\right] \times\left[r_{j}, r_{j+1}\right]\right),
\end{gathered}
$$

which tends to zero almost surely as the mesh of the partition goes to zero and is also bounded above uniformly for all partitions by the random variable (up to multiplication by a nonrandom constant)

$$
\|Y\|_{p \text {-var; }[0, T]}^{2}\|R\|_{\rho \text {-var; }[0, T]^{2}} .
$$

This is in $L^{1}(\Omega)$ by Theorem 2.25 , and thus the limit of (88) vanishes by dominated convergence theorem.

We will use Proposition 4.4 to show (87). We have

$$
\begin{aligned}
\int_{[0, T]^{2}} & \left(\int_{[0, T]^{2}}\left\langle\mathcal{D}_{u}\left(Y_{s}^{\pi}-Y_{s}\right), \mathcal{D}_{v}\left(Y_{t}^{\pi}-Y_{t}\right)\right\rangle_{\mathbb{R}^{d} \otimes \mathbb{R}^{d}} \mathrm{~d} R(u, v)\right) \mathrm{d} R(s, t) \\
= & \sum_{i, j} \int_{U_{i, j}} \int_{[0, T]^{2}}\left\langle\mathcal{D}_{u} Y_{s}-\mathcal{D}_{u} Y_{r_{i}}, \mathcal{D}_{v} Y_{t}-\mathcal{D}_{v} Y_{r_{j}}\right\rangle_{\mathbb{R}^{d} \otimes \mathbb{R}^{d}} \mathrm{~d} R(u, v) \mathrm{d} R(s, t),
\end{aligned}
$$

where $U_{i, j}:=\left[r_{i}, r_{i+1}\right] \times\left[r_{j}, r_{j+1}\right]$. With $g(s, t):=\mathcal{D}_{s} Y_{t}=\mathbb{1}_{[0, t)}(s) \tilde{g}_{1}(t) \tilde{g}_{2}(s)$, where

$$
\tilde{g}_{1}(t):=J_{t}^{\mathbf{X}}, \quad \tilde{g}_{2}(s):=\left(J_{s}^{\mathbf{X}}\right)^{-1} V\left(Y_{s}\right),
$$

we see that $g$ satisfies the conditions of Proposition 4.4 almost surely. Hence, from (79), the expression in (89) vanishes almost surely as the mesh of the partition goes to zero.

Furthermore, it is bounded above uniformly for all partitions by the random variable (up to multiplication by a nonrandom constant)

$$
\left\|\tilde{g}_{1}\right\|_{\mathcal{V}^{p} ;[0, T]}^{2}\left\|\tilde{g}_{2}\right\|_{\mathcal{V}^{p} ;[0, T]}^{2}\|R\|_{\rho \text {-var; }[0, T]^{2}}^{2} .
$$

As with the case of $\|Y\|_{p \text {-var }},\left\|J^{\mathbf{X}}\right\|_{p \text {-var }}$ and $\left\|\left(J^{\mathbf{X}}\right)^{-1}\right\|_{p \text {-var }}$ have finite moments of all orders by Theorem 2.27. This in conjunction with the fact that $V(Y)$ is bounded almost surely ensures that (90) is integrable, and thus we can apply dominated convergence theorem again to complete the proof. 
5. Appending the Riemann sum approximation to the Skorohod integral. The main purpose of this section is to show that the usual Riemann-sum approximation to the Skorohod integral can be augmented with suitably corrected secondlevel rough path terms which vanish in $L^{2}(\Omega)$ as the mesh of the partition goes to zero.

We will use $\pi(n):=\left\{t_{i}^{n}\right\}$ to denote the $n$th dyadic partition of $[0, T]$, that is, $t_{i}^{n}=\frac{i T}{2^{n}}$ for $i=0, \ldots, 2^{n}$, and $\Delta_{i}^{n}$ to denote the interval $\left[t_{i}^{n}, t_{i+1}^{n}\right]$.

In addition, $\rho^{\prime}$ will denote the Hölder conjugate of $\rho$, that is, $\frac{1}{\rho}+\frac{1}{\rho^{\prime}}=1$.

Proposition 5.1. Let $X$ be a continuous, centered Gaussian process in $\mathbb{R}^{d}$ with i.i.d. components, and for $p \in[2,4)$, let $\mathbf{X} \in \mathcal{C}^{0, p-\operatorname{var}}\left([0, T] ; G^{\lfloor p\rfloor}\left(\mathbb{R}^{d}\right)\right)$ denote the geometric rough path constructed from the limit of the piecewise-linear approximations of $X$.

Let $\rho$ and $q$ be such that $\rho \in[1,2)$ and $\frac{1}{p}+\frac{1}{q}>1$. We assume that the covariance function of $X$ satisfies:

(a) $\|R\|_{\rho \text {-var; }[0, T]^{2}}<\infty$,

(b) $\|R(t, \cdot)-R(s, \cdot)\|_{q-\operatorname{var} ;[0, T]} \leq C|t-s|^{\frac{1}{\rho}}$, for all $s, t \in[0, T]$.

Now let $\psi: \Omega \times[0, T] \rightarrow \mathbb{R}^{d} \otimes \mathbb{R}^{d}$ be a stochastic process satisfying $\psi_{t}=$ $\sum_{a, b=1}^{d} \psi_{t}^{(a, b)} \mathrm{d} e_{a} \otimes \mathrm{d} e_{b} \in \mathbb{D}^{4,2}\left(\mathbb{R}^{d} \otimes \mathbb{R}^{d}\right)$ for all $t \in[0, T]$. Furthermore, assume there exists $C<\infty$ such that for all $s, t \in[0, T]$ and $a, b=1, \ldots, d$, we have

$$
\left|\mathbb{E}\left[\psi_{s}^{(a, b)} \psi_{t}^{(a, b)}\right]\right| \leq C,
$$

and for $k=2,4$, we have

$$
\left|\mathbb{E}\left[\mathcal{D}_{h_{1}, \ldots, h_{k}}^{k}\left(\psi_{s}^{(a, b)} \psi_{t}^{(a, b)}\right)\right]\right| \leq C \prod_{i=1}^{k}\left\|\Phi\left(h_{i}\right)\right\|_{q-\operatorname{var} ;[0, T]},
$$

for all $h_{1}, \ldots, h_{k} \in \mathcal{H}_{1}^{d}$.

Then

$$
\lim _{n \rightarrow \infty}\left\|\sum_{i=0}^{2^{n}-1} \psi_{t_{i}^{n}}\left(\mathbf{X}_{t_{i}^{n}, t_{i+1}^{n}}^{2}-\frac{1}{2} \sigma^{2}\left(t_{i}^{n}, t_{i+1}^{n}\right) \mathcal{I}_{d}\right)\right\|_{L^{2}(\Omega)}=0 .
$$

ProOF. First, note that

$$
\begin{gathered}
\left\|\sum_{i=0}^{2^{n}-1} \psi_{t_{i}^{n}}\left(\mathbf{X}_{t_{i}^{n}, t_{i+1}^{n}}^{2}-\frac{1}{2} \sigma^{2}\left(t_{i}^{n}, t_{i+1}^{n}\right) \mathcal{I}_{d}\right)\right\|_{L^{2}(\Omega)} \\
\leq\left\|\sum_{i=0}^{2^{n}-1} \psi_{t_{i}^{n}}\left(\left(\mathbf{X}_{t_{i}^{n}, t_{i+1}^{n}}^{2}\right)^{S}-\frac{1}{2} \sigma^{2}\left(t_{i}^{n}, t_{i+1}^{n}\right) \mathcal{I}_{d}\right)\right\|_{L^{2}(\Omega)} \\
+\left\|\sum_{i=0}^{2^{n}-1} \psi_{t_{i}^{n}}\left(\left(\mathbf{X}_{t_{i}^{n}, t_{i+1}^{n}}^{2}\right)^{A}\right)\right\|_{L^{2}(\Omega)},
\end{gathered}
$$


where $\left(\mathbf{X}^{2}\right)^{S}$ denotes the symmetric part of $\mathbf{X}^{2}$ and $\left(\mathbf{X}^{2}\right)^{A}$ denotes the antisymmetric part. The two parts will be tackled separately, and since

$$
\left\|\sum_{i=0}^{2^{n}-1} \psi_{t_{i}^{n}}\left(\left(\mathbf{X}_{t_{i}^{n}, t_{i+1}^{n}}^{2}\right)^{A}\right)\right\|_{L^{2}(\Omega)} \leq \sum_{a, b=1}^{d}\left\|\sum_{i=0}^{2^{n}-1} \psi_{t_{i}^{n}}^{(a, b)}\left(\left(\mathbf{X}_{t_{i}^{n}, t_{i+1}^{n}}^{2}\right)^{A}\right)^{(a, b)}\right\|_{L^{2}(\Omega)},
$$

and similarly for the symmetric part, we can study the convergence of each fixed $(a, b)$ th tensor component individually. For simplicity, we will henceforth suppress the notation for the component in the superscript of $\psi$.

Let $h_{1}, h_{2}, g_{1}, g_{2} \in \mathcal{H}_{1}^{d}$ be such that $\left\langle h_{i}, g_{j}\right\rangle_{\mathcal{H}_{1}^{d}}=0$ for all $i, j=1,2$. Then from the product formula (29), we have the following identities:

$$
I_{1}\left(h_{1}\right) I_{1}\left(h_{2}\right)=I_{2}\left(h_{1} \tilde{\otimes} h_{2}\right)+\left\langle h_{1}, h_{2}\right\rangle_{\mathcal{H}_{1}^{d}}
$$

(95b) $I_{2}\left(h_{1} \tilde{\otimes} h_{2}\right) I_{2}\left(g_{1} \tilde{\otimes} g_{2}\right)=I_{4}\left(h_{1} \tilde{\otimes} h_{2} \tilde{\otimes} g_{1} \tilde{\otimes} g_{2}\right) \quad$ and

(95c) $\quad I_{2}\left(h_{1} \otimes h_{1}\right) I_{2}\left(h_{2} \otimes h_{2}\right)$

$$
=I_{4}\left(h_{1} \otimes h_{1} \tilde{\otimes} h_{2} \otimes h_{2}\right)+4 I_{2}\left(h_{1} \tilde{\otimes} h_{2}\right)\left\langle h_{1}, h_{2}\right\rangle_{\mathcal{H}_{1}^{d}}+2\left\langle h_{1}, h_{2}\right\rangle_{\mathcal{H}_{1}^{d}}^{2} .
$$

Following [28], the idea of the proof is to rewrite (94) in such a way that the summands take the form

$$
\mathbb{E}\left[\psi_{t_{i}^{n}} \psi_{t_{j}^{n}} X_{u_{1}, u_{2}}^{(a)} X_{u_{3}, u_{4}}^{(b)} X_{v_{1}, v_{2}}^{(a)} X_{v_{3}, v_{4}}^{(b)}\right]
$$

where $\left[u_{1}, u_{2}\right],\left[u_{3}, u_{4}\right] \subset\left[t_{i}^{n}, t_{i+1}^{n}\right]$ and $\left[v_{1}, v_{2}\right],\left[v_{3}, v_{4}\right] \subset\left[t_{j}^{n}, t_{j+1}^{n}\right]$, or

$$
\mathbb{E}\left[\psi_{t_{i}^{n}} \psi_{t_{j}^{n}}\left(\left(X_{t_{i}^{n}, t_{i+1}^{n}}^{(a)}\right)^{2}-\sigma^{2}\left(t_{i}^{n}, t_{i+1}^{n}\right)\right)\left(\left(X_{t_{j}^{n}, t_{j+1}^{n}}^{(a)}\right)^{2}-\sigma^{2}\left(t_{j}^{n}, t_{j+1}^{n}\right)\right)\right]
$$

as appearing in the symmetric part. After applying the identities in (95) and using the duality formula (28), (92) will be used to bound the summands.

(a) For the symmetric part of the second level rough path, we have

$$
\left(\left(\mathbf{X}_{t_{i}^{n}, t_{i+1}^{n}}^{2}\right)^{S}-\frac{1}{2} \sigma^{2}\left(t_{i}^{n}, t_{i+1}^{n}\right) \mathcal{I}_{d}\right)^{(a, b)}=\frac{1}{2}\left(X_{t_{i}^{n}, t_{i+1}^{n}}^{(a)} X_{t_{i}^{n}, t_{i+1}^{n}}^{(b)}-\delta_{a b} \sigma^{2}\left(t_{i}^{n}, t_{i+1}^{n}\right)\right) .
$$

In the case where $a=b$, we need to estimate

$$
\begin{aligned}
\mathbb{E}\left[\left(\sum_{i=0}^{2} \psi_{t_{i}^{n}}\left(\left(X_{t_{i}, t_{i+1}}^{(a)}\right)^{2}-\sigma^{2}\left(t_{i}^{n}, t_{i+1}^{n}\right)\right)\right)^{2}\right] \\
=\sum_{i, j=0}^{2^{n}-1} \mathbb{E}\left[\psi _ { t _ { i } ^ { n } } \psi _ { t _ { j } ^ { n } } \left(\left(X_{t_{i}^{n}, t_{i+1}^{n}}^{(a)}\right)^{2}\right.\right. \\
\left.\left.\quad-\sigma^{2}\left(t_{i}^{n}, t_{i+1}^{n}\right)\right)\left(\left(X_{t_{j}^{n}, t_{j+1}^{n}}^{(a)}\right)^{2}-\sigma^{2}\left(t_{j}^{n}, t_{j+1}^{n}\right)\right)\right] \\
=\sum_{i, j=0}^{2^{n}-1} \mathbb{E}\left[\psi_{t_{i}^{n}} \psi_{t_{j}^{n}} I_{2}\left(\mathbb{1}_{\Delta_{i}^{n}}^{(a)} \otimes \mathbb{1}_{\Delta_{i}^{n}}^{(a)}\right) I_{2}\left(\mathbb{1}_{\Delta_{j}^{n}}^{(a)} \otimes \mathbb{1}_{\Delta_{j}^{n}}^{(a)}\right)\right],
\end{aligned}
$$


where the last line follows from (95a). Using (95c) with $h_{1}=\mathbb{1}_{\Delta_{i}^{n}}^{(a)}(\cdot)$ and $h_{2}=$ $\mathbb{1}_{\Delta_{j}^{n}}^{(a)}(\cdot)$ and applying the duality formula (28), the expression above is equal to

$$
\begin{aligned}
& \sum_{i, j=0}^{2^{n}-1} \mathbb{E}\left[\mathcal{D}_{h_{1}, h_{1}, h_{2}, h_{2}}^{4} \psi_{t_{i}^{n}} \psi_{t_{j}^{n}}\right]+4 \mathbb{E}\left[\mathcal{D}_{h_{1}, h_{2}}^{2} \psi_{t_{i}^{n}} \psi_{t_{j}^{n}}\right] R\left(\begin{array}{cc}
t_{i}^{n} & t_{i+1}^{n} \\
t_{j}^{n} & t_{j+1}^{n}
\end{array}\right) \\
& \quad+2 \mathbb{E}\left[\psi_{t_{i}^{n}} \psi_{t_{j}^{n}}\right] R\left(\begin{array}{cc}
t_{i}^{n} & t_{i+1}^{n} \\
t_{j}^{n} & t_{j+1}^{n}
\end{array}\right) .
\end{aligned}
$$

For the first term, we have

$$
\begin{aligned}
& \sum_{i, j=0}^{2^{n}-1} \mathbb{E}\left[\mathcal{D}_{h_{1}, h_{1}, h_{2}, h_{2}}^{4} \psi_{t_{i}^{n}} \psi_{t_{j}^{n}}\right] \\
& \quad \leq C \sum_{i, j=0}^{2^{n}-1}\left\|R\left(\Delta_{i}^{n}, \cdot\right)\right\|_{q-\operatorname{var} ;[0, T]}^{2}\left\|R\left(\Delta_{j}^{n}, \cdot\right)\right\|_{q-\operatorname{var} ;[0, T]}^{2} \\
& \quad \leq \frac{C}{2^{2 n\left(\frac{2}{\rho}-1\right)}} \rightarrow 0
\end{aligned}
$$

since $\rho<2$.

For the second term, we have

$$
\sum_{i, j=0}^{2^{n}-1} \mathbb{E}\left[\mathcal{D}_{h_{1}, h_{2}}^{2} \psi_{t_{i}^{n}} \psi_{t_{j}^{n}}\right] R\left(\begin{array}{cc}
t_{i}^{n} & t_{i+1}^{n} \\
t_{j}^{n} & t_{j+1}^{n}
\end{array}\right)
$$

$$
\begin{aligned}
& \leq\left(\sum_{i, j=0}^{2^{n}-1} \mathbb{E}\left[\mathcal{D}_{h_{1}, h_{2}}^{2} \psi_{t_{i}^{n}} \psi_{t_{j}^{n}}\right]^{\rho^{\prime}}\right)^{\frac{1}{\rho^{\prime}}}\left(\sum_{i, j=0}^{2^{n}-1}\left|R\left(\begin{array}{cc}
t_{i}^{n} & t_{i+1}^{n} \\
t_{j}^{n} & t_{j+1}^{n}
\end{array}\right)\right|^{\rho}\right)^{\frac{1}{\rho}} \\
& \leq C 2^{-2 n\left(\frac{1}{\rho}-\frac{1}{\rho^{\prime}}\right)}\|R\|_{\rho \text {-var; }[0, T]^{2}}=C 2^{-2 n\left(\frac{2}{\rho}-1\right)}\|R\|_{\rho \text {-var; }[0, T]^{2}},
\end{aligned}
$$

which also vanishes as $n$ tends to infinity.

For the third term, we have

$$
\begin{aligned}
& \sum_{i, j=0}^{2^{n}-1} \mathbb{E}\left[\psi_{t_{i}^{n}} \psi_{t_{j}^{n}}\right] R\left(\begin{array}{cc}
t_{i}^{n} & t_{i+1}^{n} \\
t_{j}^{n} & t_{j+1}^{n}
\end{array}\right){ }^{2} \\
& \quad \leq C \sum_{i, j=0}^{2^{n}-1}\left|R\left(\begin{array}{cc}
t_{i}^{n} & t_{i+1}^{n} \\
t_{j}^{n} & t_{j+1}^{n}
\end{array}\right)\right|^{2-\rho}\left|R\left(\begin{array}{cc}
t_{i}^{n} & t_{i+1}^{n} \\
t_{j}^{n} & t_{j+1}^{n}
\end{array}\right)\right|^{\rho} \\
& \leq \frac{C}{2^{\frac{n(2-\rho)}{\rho}}}\|R\|_{\rho \text {-var; }[0, T]^{2}}^{\rho},
\end{aligned}
$$

which vanishes as $n \rightarrow \infty$ since $\rho<2$. 
In the case where $a \neq b$, we let

$$
h_{1}:=\mathbb{1}_{\Delta_{i}^{n}}^{(a)}(\cdot), \quad h_{2}:=\mathbb{1}_{\Delta_{j}^{n}}^{(a)}(\cdot), \quad g_{1}:=\mathbb{1}_{\Delta_{i}^{n}}^{(b)}(\cdot) \quad \text { and } \quad g_{2}:=\mathbb{1}_{\Delta_{j}^{n}}^{(b)}(\cdot) .
$$

We obtain

$$
\begin{aligned}
\mathbb{E}\left[\psi_{t_{i}^{n}}\right. & \left.\psi_{t_{j}^{n}} X_{\Delta_{i}^{n}}^{(a)} X_{\Delta_{i}^{n}}^{(b)} X_{\Delta_{j}^{n}}^{(a)} X_{\Delta_{j}^{n}}^{(b)}\right] \\
= & \mathbb{E}\left[\psi_{t_{i}^{n}} \psi_{t_{j}^{n}}\left(I_{2}\left(h_{1} \tilde{\otimes} h_{2}\right)+\left\langle h_{1}, h_{2}\right\rangle_{\mathcal{H}_{1}^{d}}\right)\left(I_{2}\left(g_{1} \tilde{\otimes} g_{2}\right)+\left\langle g_{1}, g_{2}\right\rangle_{\mathcal{H}_{1}^{d}}\right)\right] \\
= & \mathbb{E}\left[\psi_{t_{i}^{n}} \psi_{t_{j}^{n}} I_{4}\left(h_{1} \tilde{\otimes} h_{2} \tilde{\otimes} g_{1} \tilde{\otimes} g_{2}\right)\right]+\mathbb{E}\left[\psi_{t_{i}^{n}} \psi_{t_{j}^{n}} I_{2}\left(h_{1} \tilde{\otimes} h_{2}\right)\right]\left\langle g_{1}, g_{2}\right\rangle_{\mathcal{H}_{1}^{d}} \\
& +\mathbb{E}\left[\psi_{t_{i}^{n}} \psi_{t_{j}^{n}} I_{2}\left(g_{1} \tilde{\otimes} g_{2}\right)\right]\left\langle h_{1}, h_{2}\right\rangle_{\mathcal{H}_{1}^{d}}+\mathbb{E}\left[\psi_{t_{i}^{n}} \psi_{t_{j}^{n}}\right]\left\langle h_{1}, h_{2}\right\rangle_{\mathcal{H}_{1}^{d}}\left\langle g_{1}, g_{2}\right\rangle_{\mathcal{H}_{1}^{d}} \\
= & \mathbb{E}\left[\mathcal{D}_{h_{1}, h_{2}, g_{1}, g_{2}}^{4} \psi_{t_{i}^{n}} \psi_{t_{j}^{n}}\right]+\mathbb{E}\left[\psi_{t_{i}^{n}} \psi_{t_{j}^{n}}\right] R\left(\begin{array}{cc}
t_{i}^{n} & t_{i+1}^{n} \\
t_{j}^{n} & t_{j+1}^{n}
\end{array}\right)^{2} \\
& +\left(\mathbb{E}\left[\mathcal{D}_{h_{1}, h_{2}}^{2} \psi_{t_{i}^{n}} \psi_{t_{j}^{n}}\right]+\mathbb{E}\left[\mathcal{D}_{g_{1}, g_{2}}^{2} \psi_{t_{i}^{n}} \psi_{t_{j}^{n}}\right]\right) R\left(\begin{array}{cc}
t_{i}^{n} & t_{i+1}^{n} \\
t_{j}^{n} & t_{j+1}^{n}
\end{array}\right) .
\end{aligned}
$$

Similar to the case where $a=b$, the sum over all $i, j$ of the first, second and third terms in the above expression can be bounded by (97), (99) and (98) respectively, and hence vanish as $n \rightarrow \infty$.

(b) We will now handle the antisymmetric part. We will use $\left(\mathbf{X}_{s, t}^{2}\right)^{A}(\pi(k))$ to denote the Lévy area of $X^{\pi(k)}$, the piecewise linear approximation of $X$ over $\pi(k)$, that is,

$$
\left(\mathbf{X}_{s, t}^{2}\right)^{A}(\pi(k))=\pi_{2}\left(\log \left(S_{2}\left(X^{\pi(k)}\right)_{s, t}\right)\right),
$$

where $\pi_{2}$ denotes projection onto the second level. Next, we define

$$
\left(\mathbf{X}_{s, t}^{2}\right)^{A}\left(\Delta_{l+1}\right):=\left(\mathbf{X}_{s, t}^{2}\right)^{A}(\pi(l+1))-\left(\mathbf{X}_{s, t}^{2}\right)^{A}(\pi(l)),
$$

and noticing that $\left(\mathbf{X}_{t_{i}^{n}, t_{i+1}^{n}}^{2}\right)^{A}(\pi(n))=0$, we can use Theorem 2.13 to see that

$$
\begin{aligned}
\left(\mathbf{X}_{t_{i}^{n}, t_{i+1}^{n}}^{2}\right)^{A}= & \lim _{m \rightarrow \infty} \sum_{k=1}^{m}\left(\mathbf{X}_{t_{i}^{n}, t_{i+1}^{n}}^{2}\right)^{A}\left(\Delta_{n+k}\right) \\
& \text { for every } n \in \mathbb{N} \text { and } i=0,1, \ldots, 2^{n}-1,
\end{aligned}
$$

where the limit is taken in $L^{2}(\Omega)$.

We want to show that

$$
\left\|\sum_{i=0}^{2^{n}-1} \psi_{t_{i}^{n}}\left(\left(\mathbf{X}_{t_{i}^{n}, t_{i+1}^{n}}^{2}\right)^{A}(\pi(n+m))\right)^{(a, b)}\right\|_{L^{2}(\Omega)} \rightarrow 0
$$

uniformly for all $m$ as $n \rightarrow \infty$. To begin, let

$$
s_{u}^{k, i}:=t_{i}^{n}+\frac{u}{2^{n+k}}=t_{u+i 2^{k}}^{n+k},
$$


and we will denote the intervals

$$
\begin{aligned}
\Delta_{u^{L}}^{i} & :=\left[s_{2 u}^{k, i}, s_{2 u+1}^{k, i}\right], \quad \Delta_{u^{R}}^{i}:=\left[s_{2 u+1}^{k, i}, s_{2 u+2}^{k, i}\right], \\
\Delta_{u}^{i} & :=\Delta_{u^{L}}^{i} \cup \Delta_{u^{R}}^{i} \subseteq\left[t_{i}^{n}, t_{i+1}^{n}\right] \quad \forall u=0, \ldots, 2^{k-1}-1 .
\end{aligned}
$$

Note that we suppress the dependence on $k$ and $n$ in the notation for the variables on the left, and we will also use $X_{[s, t]}$ and $X_{s, t}(s, t \in[0, T])$ interchangeably. Continuing, we have

$$
\begin{aligned}
\bigotimes_{u=0}^{2^{k-1}-1} & \exp \left(X_{\Delta_{u}^{i} L}\right) \otimes \exp \left(X_{\Delta_{u}^{i} R}\right)-\bigotimes_{u=0}^{2^{k-1}-1} \exp \left(X_{\Delta_{u}^{i}}\right) \\
= & \bigotimes_{u=0}^{2^{k-1}-1}\left(1, X_{\Delta_{u}^{i}}, \frac{\left(X_{\Delta_{u}^{i}}\right)^{\otimes 2}}{2}\right)+\left(0,0, \frac{1}{2}\left[X_{\Delta_{u}^{i}{ }_{u}}, X_{\Delta_{u^{i}}^{i}}\right]\right) \\
& -\bigotimes_{u=0}^{2^{k-1}-1}\left(1, X_{\Delta_{u}^{i}}, \frac{\left(X_{\Delta_{u}^{i}}\right)^{\otimes 2}}{2}\right) \\
= & \sum_{u=0}^{2^{k-1}-1}\left(0,0, \frac{1}{2}\left[X_{\Delta_{u}^{i}{ }^{i}}, X_{\Delta_{u}^{i}}\right]\right),
\end{aligned}
$$

which means that

$$
\left(\mathbf{X}_{t_{i}^{n}, t_{i+1}^{n}}^{2}\right)^{A}\left(\Delta_{n+k}\right)=\sum_{u=0}^{2^{k-1}-1} \frac{1}{2}\left[X_{\Delta_{u}^{i} L}, X_{\Delta_{u}^{i} R}\right]
$$

since only antisymmetric terms are left in the difference.

Thus, we obtain

$$
\begin{aligned}
\mathbb{E}\left[\left(\sum_{i=0}^{2^{n}-1} \psi_{t_{i}^{n}}\left(\left(\mathbf{X}_{t_{i}^{n}, t_{i+1}^{n}}^{2}\right)^{A}(\pi(n+m))\right)^{(a, b)}\right)^{2}\right] \\
=\mathbb{E}\left[\left(\sum_{i=0}^{2^{n}-1} \psi_{t_{i}^{n}} \sum_{k=1}^{m}\left(\left(\mathbf{X}_{t_{i}^{n}, t_{i+1}^{n}}^{2}\right)^{A}\left(\Delta_{n+k}\right)\right)^{(a, b)}\right)^{2}\right] \\
=\sum_{i, j=0}^{2^{n}-1} \mathbb{E}\left[\psi_{t_{i}^{n}} \psi_{t_{j}^{n}} \sum_{k=1}^{m}\left(\left(\mathbf{X}_{t_{i}^{n}, t_{i+1}^{n}}^{2}\right)^{A}\left(\Delta_{n+k}\right)\right)^{(a, b)}\right. \\
\left.\quad \times \sum_{l=1}^{m}\left(\left(\mathbf{X}_{t_{j}^{n}, t_{j+1}^{n}}^{2}\right)^{A}\left(\Delta_{n+l}\right)\right)^{(a, b)}\right] \\
=\frac{1}{4} \sum_{i, j=0}^{2^{n}-1} \sum_{k, l=1}^{m} \sum_{u, v} \mathbb{E}\left[\psi_{t_{i}^{n}} \psi_{t_{j}^{n}}\left[X_{\Delta_{u}^{i} L}, X_{\Delta_{u}^{i} R}\right]^{(a, b)}\left[X_{\Delta_{v^{L}}^{j}}, X_{\Delta_{v^{R}}^{j}}\right]^{(a, b)}\right] .
\end{aligned}
$$


Since the $(a, b)$ th entry of $\left[X_{\Delta_{u}^{i}{ }^{i}}, X_{\Delta_{u^{R}}^{i}}\right] \in s o(d)$, where $a \neq b$, is given by

$$
X_{\Delta_{{ }^{i} L}^{i}}^{(a)} X_{\Delta_{{ }^{i} R}^{i}}^{(b)}-X_{\Delta_{{ }^{i} L}^{i}}^{(b)} X_{\Delta_{{ }^{i} R}^{i}}^{(a)}
$$

each summand in the last line of (102) is of the form

$$
\begin{aligned}
& \mathbb{E}\left[\psi_{t_{i}^{n}} \psi_{t_{j}^{n}} X_{\Delta_{u^{L}}^{i}}^{(a)} X_{\Delta_{u^{i}}^{i}}^{(b)} X_{\Delta_{v^{j}}^{j}}^{(a)} X_{\Delta_{v^{j}}{ }^{j}}^{(b)}\right] \\
& -\mathbb{E}\left[\psi_{t_{i}^{n}} \psi_{t_{j}^{n}} X_{\Delta_{u^{L}}^{i}}^{(a)} X_{\Delta_{u^{i}}^{i}}^{(b)} X_{\Delta_{v^{j}}^{j}}^{(b)} X_{\Delta_{v^{j}}{ }^{j}}^{(a)}\right] \\
& -\mathbb{E}\left[\psi_{t_{i}^{n}} \psi_{t_{j}^{n}} X_{\Delta_{u^{L}}^{i}}^{(b)} X_{\Delta_{u^{i}}^{i}}^{(a)} X_{\Delta_{v^{j}}^{j}}^{(a)} X_{\Delta_{v}^{j}{ }^{j}}^{(b)}\right] \\
& +\mathbb{E}\left[\psi_{t_{i}^{n}} \psi_{t_{j}^{n}} X_{\Delta_{u^{i} L}^{(b)}}^{(b)} X_{\Delta_{u^{i} R}^{(a)}}^{(a)} X_{\Delta_{v^{L}}{ }^{j}}^{(b)} X_{\Delta_{v^{j}}^{j}}^{(a)}\right] .
\end{aligned}
$$

Proceeding, we will use the first term in the above expression for the proof and omit the other terms as the result remains the same with trivial modifications to the notation. We first denote

$$
\begin{aligned}
R_{\Delta_{u}^{i} \times \Delta_{v}^{j}}:= & \left|R\left(\begin{array}{cc}
s_{2 u}^{k, i} & s_{2 u+1}^{k, i} \\
l, j & l, j \\
s_{2 v} & s_{2 v+1}^{l, j}
\end{array}\right)\right|+\left|R\left(\begin{array}{cc}
s_{2 u+1}^{k, i} & s_{2 u+2}^{k, i} \\
l, j & l, j \\
s_{2 v}^{l, j} & s_{2 v+1}
\end{array}\right)\right| \\
& +\left|R\left(\begin{array}{cc}
s_{2 u}^{k, i} & s_{2 u+1}^{k, i} \\
s_{2 v+1}^{l, j} & s_{2 v+2}^{l, j}
\end{array}\right)\right|+\left|R\left(\begin{array}{cc}
s_{2 u+1}^{k, i} & s_{2 u+2}^{k, i} \\
l, j & l, j \\
s_{2 v+1}^{l, j} & s_{2 v+2}^{l, j}
\end{array}\right)\right|,
\end{aligned}
$$

and note that

$$
\sum_{i, j=0}^{2^{n}-1} \sum_{u, v} R_{\Delta_{u}^{i} \times \Delta_{v}^{j}}^{\rho} \leq 4^{\rho}\|R\|_{\rho \text {-var; }[0, T]^{2}}^{\rho} .
$$

Next, we let

$$
h_{1}:=\mathbb{1}_{\Delta_{u^{L}}^{i}}^{(a)}(\cdot), \quad h_{2}:=\mathbb{1}_{\Delta_{v^{L}}^{j}}^{(a)}(\cdot), \quad g_{1}:=\mathbb{1}_{\Delta_{{ }^{i} R}^{i}}^{(b)}(\cdot) \quad \text { and } \quad g_{2}:=\mathbb{1}_{\Delta_{v^{R}}^{j}}^{(b)}(\cdot),
$$

and applying (95b), we get

$$
\begin{aligned}
\mathbb{E}\left[\psi_{t_{i}^{n}}\right. & \left.\psi_{t_{j}^{n}} X_{\Delta_{u}^{i} L}^{(a)} X_{\Delta_{u}^{i} R}^{(b)} X_{\Delta_{v^{j}}^{j}}^{(a)} X_{\Delta_{v^{j}}^{j}}^{(b)}\right] \\
= & \mathbb{E}\left[\psi_{t_{i}^{n}} \psi_{t_{j}^{n}}\left(I_{2}\left(h_{1} \tilde{\otimes} h_{2}\right)+\left\langle h_{1}, h_{2}\right\rangle_{\mathcal{H}_{1}^{d}}\right)\left(I_{2}\left(g_{1} \tilde{\otimes} g_{2}\right)+\left\langle g_{1}, g_{2}\right\rangle_{\mathcal{H}_{1}^{d}}\right)\right] \\
= & \mathbb{E}\left[\psi_{t_{i}^{n}} \psi_{t_{j}^{n}} I_{4}\left(h_{1} \tilde{\otimes} h_{2} \tilde{\otimes} g_{1} \tilde{\otimes} g_{2}\right)\right]+\mathbb{E}\left[\psi_{t_{i}^{n}} \psi_{t_{j}^{n}} I_{2}\left(h_{1} \tilde{\otimes} h_{2}\right)\right]\left\langle g_{1}, g_{2}\right\rangle_{\mathcal{H}_{1}^{d}} \\
& +\mathbb{E}\left[\psi_{t_{i}^{n}} \psi_{t_{j}^{n}} I_{2}\left(g_{1} \tilde{\otimes} g_{2}\right)\right]\left\langle h_{1}, h_{2}\right\rangle_{\mathcal{H}_{1}^{d}}+\mathbb{E}\left[\psi_{t_{i}^{n}} \psi_{t_{j}^{n}}\right]\left\langle h_{1}, h_{2}\right\rangle_{\mathcal{H}_{1}^{d}}\left\langle g_{1}, g_{2}\right\rangle_{\mathcal{H}_{1}^{d}} \\
= & : A_{1}+A_{2}+A_{3}+A_{4} .
\end{aligned}
$$


(i) Terms of type $A_{1}$ :

$$
\begin{aligned}
&\left|\mathbb{E}\left[\mathcal{D}_{h_{1}, h_{2}, g_{1}, g_{2}}^{4}\left(\psi_{t_{i}^{n}} \psi_{t_{j}^{n}}\right)\right]\right| \\
& \leq C\left\|\Phi\left(h_{1}\right)\right\|_{q \text {-var; }[0, T]}\left\|\Phi\left(h_{2}\right)\right\|_{q \text {-var; }[0, T]} \\
& \times\left\|\Phi\left(g_{1}\right)\right\|_{q \text {-var; }[0, T]}\left\|\Phi\left(g_{2}\right)\right\|_{q \text {-var; }[0, T]} \\
& \leq C\left\|R\left(s_{2 u+1}^{k, i}, \cdot\right)-R\left(s_{2 u}^{k, i}, \cdot\right)\right\|_{q \text {-var; }[0, T]} \\
& \times\left\|R\left(s_{2 v+1}^{l, j}, \cdot\right)-R\left(s_{2 v}^{l, j}, \cdot\right)\right\|_{q \text {-var; }[0, T]} \\
& \times\left\|R\left(s_{2 u+2}^{k, i}, \cdot\right)-R\left(s_{2 u+1}^{k, i}, \cdot\right)\right\|_{q \text {-var; }[0, T]} \\
& \times\left\|R\left(s_{2 v+2}^{l, j}, \cdot\right)-R\left(s_{2 v+1}^{l, j}, \cdot\right)\right\|_{q \text {-var; }[0, T]} \\
& \leq C 2^{\frac{-2(n+k)}{\rho}} 2^{\frac{-2(n+l)}{\rho}},
\end{aligned}
$$

and thus we have

$$
\begin{aligned}
& \sum_{i, j=0}^{2^{n}-1} \sum_{k, l=1}^{m} \sum_{u, v}\left|\mathbb{E}\left[\mathcal{D}_{h_{1}, h_{2}, g_{1}, g_{2}}^{4}\left(\psi_{t_{i}^{n}} \psi_{t_{j}^{n}}\right)\right]\right| \\
& \leq C 2^{-2 n\left(\frac{2}{\rho}-1\right)} \sum_{k, l=1}^{\infty} 2^{-k\left(\frac{2}{\rho}-1\right)} 2^{-l\left(\frac{2}{\rho}-1\right)} \\
& \leq C 2^{-2 n\left(\frac{2}{\rho}-1\right)} \rightarrow 0 \quad \text { as } n \rightarrow \infty
\end{aligned}
$$

(ii) Terms of type $A_{2}$ and $A_{3}$ : We only detail the argument for the $A_{2}$ terms; the $A_{3}$ terms can be dealt with in the same way. Using Hölder's inequality and by exploiting the upper bound,

$$
\left|\mathbb{E}\left[\mathcal{D}_{h_{1}, h_{2}}^{2}\left(\psi_{t_{i}^{n}} \psi_{t_{j}^{n}}\right)\right]\right| \leq C\left|s_{2 u+1}^{k, i}-s_{2 u}^{k, i}\right|^{\frac{1}{\rho}}\left|s_{2 v+1}^{l, j}-s_{2 v}^{l, j}\right|^{\frac{1}{\rho}} \leq C 2^{-\frac{(n+k)}{\rho}} 2^{-\frac{(n+l)}{\rho}},
$$

we obtain

$$
\begin{aligned}
& \sum_{i, j=0}^{2^{n}-1} \sum_{k, l=1}^{m} \sum_{u, v} \mathbb{E}\left[\mathcal{D}_{h_{1}, h_{2}}^{2}\left(\psi_{t_{i}^{n}} \psi_{t_{j}^{n}}\right)\right] \mathbb{E}\left[X_{\Delta_{u}^{i} R}^{(b)} X_{\Delta_{v^{j}}^{j}}^{(b)}\right] \\
& \leq C \sum_{k, l=1}^{m}\left(\sum_{i, j=0}^{2^{n}-1} \sum_{u, v} \mid \mathbb{E}\left[\left.\mathcal{D}_{h_{1}, h_{2}}^{2}\left(\psi_{t_{i}^{n}} \psi_{\left.t_{j}^{n}\right)}\right]\right|^{\rho^{\prime}}\right)^{\frac{1}{\rho^{\prime}}}\left(\sum_{i, j=0}^{2^{n}-1} \sum_{u, v} R_{\Delta_{u}^{i} \times \Delta_{v}^{j}}^{\rho}\right)^{\frac{1}{\rho}}\right. \\
& \leq C \sum_{k, l=1}^{\infty} 2^{-2 n\left(\frac{1}{\rho}-\frac{1}{\rho^{\prime}}\right)} 2^{-k\left(\frac{1}{\rho}-\frac{1}{\rho^{\prime}}\right)} 2^{-l\left(\frac{1}{\rho}-\frac{1}{\rho^{\prime}}\right)}\|R\|_{\rho-\operatorname{var} ;[0, T]^{2}}
\end{aligned}
$$


Since $\frac{1}{\rho}-\frac{1}{\rho^{\prime}}=\frac{2}{\rho}-1>0$, we may sum over $k$ and $l$ to get

$$
\sum_{i, j=0}^{2^{n}-1} \sum_{k, l=1}^{m} \sum_{u, v} \mathbb{E}\left[\mathcal{D}_{h_{1}, h_{2}}^{2}\left(\psi_{t_{i}^{n}} \psi_{t_{j}^{n}}\right)\right] \mathbb{E}\left[X_{\Delta_{u}^{i} R}^{(b)} X_{\Delta_{v R}^{j}}^{(b)}\right] \leq C 2^{-2 n\left(\frac{2}{\rho}-1\right)}\|R\|_{\rho \text {-var; }[0, T]^{2},}
$$

which tends to zero as $n \rightarrow \infty$.

(iii) Terms of type $A_{4}$ : We have

$$
\begin{aligned}
& \mathbb{E}\left[\psi_{t_{i}^{n}} \psi_{t_{j}^{n}}\right]\left\langle h_{1}, h_{2}\right\rangle_{\mathcal{H}_{1}^{d}}\left\langle g_{1}, g_{2}\right\rangle_{\mathcal{H}_{1}^{d}} \\
& \quad=\mathbb{E}\left[\psi_{t_{i}^{n}} \psi_{t_{j}^{n}}\right] R\left(\begin{array}{cc}
s_{2 u}^{k, i} & s_{2 u+1}^{k, i} \\
l, j & l, j, j \\
s_{2 v} & s_{2 v+1}^{l, j}
\end{array}\right) R\left(\begin{array}{cc}
s_{2 u+1}^{k, i} & s_{2 u+2}^{k, i} \\
l, j & l, j \\
s_{2 v+1}^{l, j} & s_{2 v+2}^{l, j}
\end{array}\right) \\
& \leq C R_{\Delta_{u}^{i} \times \Delta_{v}^{j}}^{2} .
\end{aligned}
$$

Using the fact that

$$
\begin{aligned}
R_{\Delta_{u}^{i} \times \Delta_{v}^{j}} \leq & 2\left\|R\left(s_{2 u+1}^{k, i}, \cdot\right)-R\left(s_{2 u}^{k, i}, \cdot\right)\right\|_{q-\operatorname{var} ;[0, T]} \\
& +2\left\|R\left(s_{2 u+2}^{k, i}, \cdot\right)-R\left(s_{2 u+1}^{k, i}, \cdot\right)\right\|_{q \text {-var; }[0, T]}
\end{aligned}
$$

and

$$
\begin{aligned}
R_{\Delta_{u}^{i} \times \Delta_{v}^{j}} \leq & 2\left\|R\left(s_{2 v+1}^{l, j}, \cdot\right)-R\left(s_{2 v}^{l, j}, \cdot\right)\right\|_{q-\operatorname{var} ;[0, T]} \\
& +2\left\|R\left(s_{2 v+2}^{l, j}, \cdot\right)-R\left(s_{2 v+1}^{l, j}, \cdot\right)\right\|_{q-\operatorname{var} ;[0, T]}
\end{aligned}
$$

we have

$$
\begin{aligned}
\sum_{k, l=1}^{m} \sum_{i, j=0}^{2^{n}-1} \sum_{u, v} R_{\Delta_{u}^{i} \times \Delta_{v}^{j}}^{2} & \leq C \sum_{k, l=1}^{m} 2^{-(n+k) \frac{2-\rho}{2 \rho}} 2^{-(n+l) \frac{2-\rho}{2 \rho}} \sum_{i, j=0}^{2^{n}-1} \sum_{u, v} R_{\Delta_{u}^{i} \times \Delta_{v}^{j}}^{\rho} \\
& \leq C 2^{-2 n\left(\frac{1}{\rho}-\frac{1}{2}\right)} \sum_{k, l=1}^{\infty} 2^{-k\left(\frac{1}{\rho}-\frac{1}{2}\right)} 2^{-l\left(\frac{1}{\rho}-\frac{1}{2}\right)}\|R\|_{\rho-\operatorname{var} ;[0, T]^{2}}^{\rho},
\end{aligned}
$$

which converges to 0 since $\frac{1}{\rho}-\frac{1}{2}>0$.

Given the preceding proposition, the following corollary is straightforward.

Corollary 5.2. For $2 \leq p<4$, let $Y \in \mathcal{C}^{p \text {-var }}\left([0, T] ; \mathbb{R}^{d}\right)$ denote the pathlevel solution to

$$
\mathrm{d} Y_{t}=V\left(Y_{t}\right) \circ \mathrm{d} \mathbf{X}_{t}, \quad Y_{0}=y_{0},
$$

where $\mathbf{X} \in \mathcal{C}^{0, p-\operatorname{var}}\left([0, T] ; G^{\lfloor p\rfloor}\left(\mathbb{R}^{d}\right)\right)$ satisfies the same conditions as in Proposition 5.1. Then if $V \in \mathcal{C}_{b}^{\lfloor p\rfloor+4}\left(\mathbb{R}^{d} ; \mathbb{R}^{d} \otimes \mathbb{R}^{d}\right)$, we have

$$
\lim _{\|\pi(n)\| \rightarrow 0} \| \sum_{i} V\left(Y_{\left.t_{i}^{n}\right)}\left(\mathbf{X}_{t_{i}^{n}, t_{i+1}^{n}}^{2}-\frac{1}{2} \sigma^{2}\left(t_{i}^{n}, t_{i+1}^{n}\right) \mathcal{I}_{d}\right) \|_{L^{2}(\Omega)}=0 .\right.
$$


Proof. Since $V \in \mathcal{C}_{b}^{1},\left|\mathbb{E}\left[V_{s}^{(a, b)} V_{t}^{(a, b)}\right]\right|$ is bounded for all $s, t, a$ and $b$. Now we have to show that bound (92) in Proposition 5.1 is satisfied with

$$
\psi_{t}=V\left(Y_{t}\right) \in \mathbb{R}^{d} \otimes \mathbb{R}^{d},
$$

to show (105). To do so, recall Proposition 3.5, which states that almost surely we have

$$
\begin{aligned}
& \left\|\mathcal{D}_{h_{1}, \ldots, h_{n}}^{n} Y \cdot\right\|_{\infty} \\
& \quad \leq P_{d(n)}\left(\|\mathbf{X}\|_{p-\operatorname{var} ;[0, T]}, \exp \left(C N_{1 ;[0, T]}^{\mathbf{X}}\right)\right) \prod_{i=1}^{n}\left\|\Phi\left(h_{i}\right)\right\|_{q-\operatorname{var} ;[0, T]} .
\end{aligned}
$$

As both $\|\mathbf{X}\|_{p \text {-var; }[0, T]}$ and $\exp \left(C N_{1 ;[0, T]}^{\mathbf{X}}\right)$ belong to $\bigcap_{r>0} L^{r}(\Omega)$, we have

$$
\left\|\mathcal{D}_{h_{1}, \ldots, h_{n}}^{n} Y_{t}\right\|_{L^{r}(\Omega)} \leq C_{n, q} \prod_{i=1}^{n}\left\|\Phi\left(h_{i}\right)\right\|_{q-\operatorname{var} ;[0, T]}
$$

for any $r>0$. Now we simply use the product and chain rule of Malliavin differentiation in conjunction with the fact that $V$ has bounded derivatives up to the appropriate order.

6. Conversion formula. We are now ready to prove the main result of the paper. As before, $\pi(n):=\left\{t_{i}^{n}\right\}, t_{i}^{n}:=\frac{i T}{2^{n}}$, denotes the sequence of dyadic partitions on $[0, T]$.

THEOREM 6.1. For $1 \leq p<3$, let $Y \in \mathcal{C}^{p \text {-var }}\left([0, T] ; \mathbb{R}^{d}\right)$ denote the pathlevel solution to

$$
\mathrm{d} Y_{t}=V\left(Y_{t}\right) \circ \mathrm{d} \mathbf{X}_{t}, \quad Y_{0}=y_{0},
$$

where $\mathbf{X} \in \mathcal{C}^{0, p \text {-var }}\left([0, T] ; G^{\lfloor p\rfloor}\left(\mathbb{R}^{d}\right)\right)$ denotes the geometric rough path constructed from the limit of the piecewise-linear approximations of $X$, a continuous, centered Gaussian process in $\mathbb{R}^{d}$ with i.i.d. components and continuous covariance function satisfying $\|R\|_{\rho \text {-var; }[0, T]^{2}}<\infty$ for some $\rho \in\left[1, \frac{3}{2}\right)$. In addition, we have the following assumptions:

(i) If $1 \leq p<2$, assume $V \in \mathcal{C}_{b}^{2}\left(\mathbb{R}^{d} ; \mathbb{R}^{d} \otimes \mathbb{R}^{d}\right), \sigma^{2}(s, t) \leq C|t-s|^{\theta}$ for some $\theta>1$ and $\|R(\cdot)\|_{q-\operatorname{var} ;[0, T]}<\infty$, where $\frac{1}{p}+\frac{1}{q}>1$.

(ii) If $2 \leq p<3$, assume that $V \in \mathcal{C}_{b}^{6}\left(\mathbb{R}^{d} ; \mathbb{R}^{d} \otimes \mathbb{R}^{d}\right)$, and the covariance function satisfies

$$
\|R(t, \cdot)-R(s, \cdot)\|_{\rho \text {-var; }[0, T]} \leq C|t-s|^{\frac{1}{\rho}},
$$

for all $s, t \in[0, T]$. 
In either case, almost surely we have

$$
\begin{aligned}
\int_{0}^{T} Y_{t} \circ \mathrm{d} \mathbf{X}_{t}= & \int_{0}^{T} Y_{t} \mathrm{~d} X_{t}+\frac{1}{2} \int_{0}^{T} \operatorname{tr}\left[V\left(Y_{s}\right)\right] \mathrm{d} R(s) \\
& +\int_{[0, T]^{2}} \mathbb{1}_{[0, t)}(s) \operatorname{tr}\left[J_{t \leftarrow s}^{\mathbf{X}} V\left(Y_{s}\right)-V\left(Y_{t}\right)\right] \mathrm{d} R(s, t) .
\end{aligned}
$$

PROOF. Using regular Riemann-Stieltjes integration when $1 \leq p<2$ and Theorem 2.20 when $2 \leq p<3, \int_{0}^{T} Y_{t} \circ \mathrm{d} \mathbf{X}_{t}$ is equal almost surely to

$$
\lim _{n \rightarrow \infty} \sum_{i} \begin{cases}Y_{t_{i}^{n}}\left(X_{t_{i}^{n}, t_{i+1}^{n}}\right), & 1 \leq p<2, \\ Y_{t_{i}^{n}}\left(X_{t_{i}^{n}, t_{i+1}^{n}}\right)+V\left(Y_{t_{i}^{n}}\right)\left(\mathbf{X}_{t_{i}^{n}, t_{i+1}^{n}}^{2}\right), & 2 \leq p<3 .\end{cases}
$$

We now apply Proposition 4.10 in conjunction with Corollary 5.2. Upon extracting a subsequence (and reusing the index for notational simplicity), the Skorohod integral is given almost surely by

$$
\int_{0}^{T} Y_{t} \mathrm{~d} X_{t}=\lim _{n \rightarrow \infty} \sum_{i}\left[Y_{t_{i}^{n}}\left(X_{t_{i}^{n}, t_{i+1}^{n}}\right)-\int_{0}^{t_{i}^{n}} \operatorname{tr}\left[J_{t_{i}^{n} \leftarrow s}^{\mathbf{X}} V\left(Y_{s}\right)\right] R\left(\Delta_{i}^{n}, \mathrm{~d} s\right)+A^{(i)}\right],
$$

where

$$
A^{(i)}:= \begin{cases}V\left(Y_{t_{i}^{n}}\right)\left(-\frac{1}{2} \sigma^{2}\left(t_{i}^{n}, t_{i+1}^{n}\right) \mathcal{I}_{d}\right), & 1 \leq p<2 \\ V\left(Y_{t_{i}^{n}}\right)\left(\left(\mathbf{X}_{t_{i}^{n}, t_{i+1}^{n}}^{2}\right)-\frac{1}{2} \sigma^{2}\left(t_{i}^{n}, t_{i+1}^{n}\right) \mathcal{I}_{d}\right), & 2 \leq p<3\end{cases}
$$

Note that when $1 \leq p<2$, we can append $\sum_{i} V\left(Y_{t_{i}^{n}}\right)\left(-\frac{1}{2} \sigma^{2}\left(t_{i}^{n}, t_{i+1}^{n}\right) \mathcal{I}_{d}\right)$ to the Riemann sum approximants of the Skorohod integral because

$$
\sum_{i} \operatorname{tr}\left[V\left(Y_{t_{i}^{n}}\right)\right] \sigma^{2}\left(t_{i}^{n}, t_{i+1}^{n}\right) \leq C \sum_{i}\left|t_{i+1}^{n}-t_{i}^{n}\right|^{\theta}
$$

which vanishes as $n \rightarrow \infty$.

In both cases, subtracting the two integrals gives us

$$
\begin{aligned}
& \int_{0}^{T} Y_{t} \circ \mathrm{d} \mathbf{X}_{t}-\int_{0}^{T} Y_{t} \mathrm{~d} X_{t} \\
& \quad=\lim _{n \rightarrow \infty} \sum_{i} \int_{0}^{t_{i}^{n}} \operatorname{tr}\left[J_{t_{i}^{n} \leftarrow s}^{\mathbf{X}} V\left(Y_{S}\right)\right] R\left(\Delta_{i}^{n}, \mathrm{~d} s\right)+\frac{1}{2} \sigma^{2}\left(t_{i}^{n}, t_{i+1}^{n}\right) \operatorname{tr}\left[V\left(Y_{t_{i}^{n}}\right)\right] .
\end{aligned}
$$

Subtracting $\operatorname{tr}\left[V\left(Y_{t_{i}^{n}}\right)\right] R\left(\Delta_{i}^{n}, t_{i}^{n}\right)$ from the first term on the right of (110) and adding it to the second term gives us

$$
\int_{0}^{t_{i}^{n}} \operatorname{tr}\left[J_{t_{i}^{n} \leftarrow s}^{\mathbf{X}} V\left(Y_{s}\right)\right] R\left(\Delta_{i}^{n}, \mathrm{~d} s\right)+\frac{1}{2} \sigma^{2}\left(t_{i}^{n}, t_{i+1}^{n}\right) \operatorname{tr}\left[V\left(Y_{t_{i}^{n}}\right)\right]=F^{(i)}+G^{(i)},
$$


where

$$
\begin{aligned}
& F^{(i)}:=\int_{0}^{t_{i}^{n}} \operatorname{tr}\left[J_{t_{i}^{n} \leftarrow s}^{\mathbf{X}} V\left(Y_{s}\right)\right] R\left(\Delta_{i}^{n}, \mathrm{~d} s\right)-\operatorname{tr}\left[V\left(Y_{t_{i}^{n}}\right)\right] R\left(\Delta_{i}^{n}, t_{i}^{n}\right), \\
& G^{(i)}:=\frac{1}{2} \sigma^{2}\left(t_{i}^{n}, t_{i+1}^{n}\right) \operatorname{tr}\left[V\left(Y_{t_{i}^{n}}\right)\right]+\operatorname{tr}\left[V\left(Y_{t_{i}^{n}}\right)\right] R\left(\Delta_{i}^{n}, t_{i}^{n}\right) .
\end{aligned}
$$

We have

$$
\begin{aligned}
F^{(i)} & =\int_{0}^{t_{i}^{n}} \operatorname{tr}\left[J_{t_{i}^{n} \leftarrow s}^{\mathbf{X}} V\left(Y_{s}\right)-V\left(Y_{t_{i}^{n}}\right)\right] R\left(\Delta_{i}^{n}, \mathrm{~d} s\right) \\
& =\int_{0}^{T} h\left(s, t_{i}^{n}\right) R\left(\Delta_{i}^{n}, \mathrm{~d} s\right),
\end{aligned}
$$

where we denote

$$
h(s, t):=\mathbb{1}_{[0, t)}(s) \operatorname{tr}\left[J_{t \leftarrow s}^{\mathbf{X}} V\left(Y_{s}\right)-V\left(Y_{t}\right)\right] .
$$

Since $h(s, t)$ vanishes on the diagonal, it is continuous almost surely on $[0, T]^{2}$. Furthermore, we have complementary regularity since $\frac{1}{p}+\frac{1}{\rho}>1$, in which case Theorem 2.12 tells us that

$$
\int_{[0, T]^{2}} h(s, t) \mathrm{d} R(s, t)
$$

exists. Thus, we have some partition $\pi^{\prime}=\left\{s_{k}\right\} \times\left\{t_{i}^{n}\right\}$ such that

$$
\left|\int_{[0, T]^{2}} h(s, t) \mathrm{d} R(s, t)-\sum_{i, k} h\left(s_{k}, t_{i}^{n}\right) R\left(\begin{array}{cc}
s_{k} & s_{k+1} \\
t_{i}^{n} & t_{i+1}^{n}
\end{array}\right)\right|<\frac{\varepsilon}{2} .
$$

Refining $\left\{s_{k}\right\}$ if necessary, we also have for each $i$

$$
\left|\int_{0}^{T} h\left(s, t_{i}^{n}\right) R\left(\Delta_{i}^{n}, \mathrm{~d} s\right)-\sum_{k} h\left(s_{k}, t_{i}^{n}\right) R\left(\begin{array}{cc}
s_{k} & s_{k+1} \\
t_{i}^{n} & t_{i+1}^{n}
\end{array}\right)\right|<\frac{\varepsilon}{2}\left(\frac{1}{2^{n}}\right),
$$

and note that these estimates hold for all $\pi=\pi_{1} \times \pi_{2}$ where $\|\pi\| \leq\left\|\pi^{\prime}\right\|$ and $\left\|\pi_{2}\right\| \leq\|\pi(n)\|$. Thus

$$
\sum_{i} F^{(i)} \rightarrow \int_{[0, T]^{2}} h(s, t) \mathrm{d} R(s, t) .
$$

For the $G$ terms, we have

$$
\begin{aligned}
\sum_{i} G^{(i)} & =\sum_{i} \operatorname{tr}\left[V\left(Y_{t_{i}^{n}}\right)\right]\left(R\left(t_{i+1}^{n}, t_{i}^{n}\right)-R\left(t_{i}^{n}, t_{i}^{n}\right)+\frac{1}{2} \sigma^{2}\left(t_{i}^{n}, t_{i+1}^{n}\right)\right) \\
& =\frac{1}{2} \sum_{i} \operatorname{tr}\left[V\left(Y_{t_{i}^{n}}\right)\right]\left(R\left(t_{i+1}^{n}, t_{i+1}^{n}\right)-R\left(t_{i}^{n}, t_{i}^{n}\right)\right),
\end{aligned}
$$


which converges to $\frac{1}{2} \int_{0}^{T} \operatorname{tr}\left[V\left(Y_{t}\right)\right] \mathrm{d} R(t)$ as $Y$ and $R(\cdot)$ have complementary regularity.

The limit in (110) necessarily exists almost surely because it is the difference of almost sure convergent sequences. However, we add and subtract $\operatorname{tr}\left[V\left(Y_{t_{i}^{n}}\right)\right] R\left(\Delta_{i}^{n}, t_{i}^{n}\right)$ because in general, if considered separately, neither term can be expected to be a convergent sequence.

Consider the case when $R(s, t)$ is the covariance function of fractional Brownian motion where $\frac{1}{3}<H<\frac{1}{2}$. For the first term of (110), formally one would expect convergence to the Young integral

$$
\int_{[0, T]^{2}} \mathbb{1}_{[0, t)}(s) \operatorname{tr}\left[J_{t \leftarrow s}^{\mathbf{X}} V\left(Y_{S}\right)\right] \mathrm{d} R(s, t)
$$

since we have complementary regularity. However, the discontinuity of the integrand at the diagonal poses a problem, as can be illustrated by the following simple example; if we take the sequence of square partitions $\left\{\left(t_{i}^{n}, t_{j}^{n}\right)\right\}$, the RiemannStieltjes sums of $\int_{[0, T]^{2}} \mathbb{1}_{[0, t)}(s) \mathrm{d} R(s, t)$ are given by

$$
\sum_{j} \sum_{i<j} R\left(\Delta_{i}, \Delta_{j}\right)=\sum_{j} R\left(t_{j}^{n}, t_{j+1}^{n}\right)-R\left(t_{j}^{n}, t_{j}^{n}\right) \rightarrow-\infty
$$

and thus $\int_{[0, T]^{2}} \mathbb{1}_{[0, t)}(s) \mathrm{d} R(s, t)$ does not exist as a Young-Stieltjes integral. For the second term of (110), if $V$ is bounded from below, we have

$$
\sum_{i} \frac{1}{2} \sigma^{2}\left(t_{i}^{n}, t_{i+1}^{n}\right) \operatorname{tr}\left[V\left(Y_{t_{i}^{n}}\right)\right] \geq C \sum_{i}\left|t_{i+1}^{n}-t_{i}^{n}\right|^{2 H},
$$

which also diverges.

Now consider the following theorem from [12].

THEOREM 6.2. Let $X$ be fractional Brownian motion with Hurst parameter $H<\frac{1}{2}$.

If $u \in \mathbb{D}^{1,2}\left(I_{0^{+}}^{\frac{3}{2}-H}\left(L^{2}\right)\right)$, then $\delta^{X}\left(\mathcal{K}^{*} u\right)$ and trace $(\mathcal{D} u)$ are well defined, and the sequence

$$
\sum_{i} \frac{1}{t_{i+1}-t_{i}} \int_{t_{i}}^{t_{i+1}} u_{t} \mathrm{~d} t\left(X_{t_{i+1}}-X_{t_{i}}\right)
$$

converges in $L^{2}(\Omega)$ to $\delta^{X}\left(\mathcal{K}^{*} u\right)+\operatorname{trace}(\mathcal{D} u)$.

Formally, $\mathcal{K}^{*}$ is the operator $K^{*} \circ D_{T^{-}}^{1}$, where $D_{T^{-}}^{1}$ is the adjoint of the derivative operator; see [13]. It is well known that the Besov-Liouville space $I_{0^{+}}^{\frac{3}{2}-H}\left(L^{2}\right)$ can be embedded continuously in $\mathcal{C}^{0,1-H}$ (see $\left.[13,29,34]\right)$, the space of $(1-H)$ 
Hölder continuous paths starting at zero. This imposes a strong condition on the integrand as one essentially requires Young-complementary regularity of $u$ and $X$.

Thus, when the integrand solves an RDE, Theorem 6.1 extends this theorem to cases where the integrand and integrator do not have complementary regularity. Furthermore, when $1 \leq p<2$, although $\int_{0}^{t_{i}^{n}} \operatorname{tr}\left[J_{t_{i}^{n} \leftarrow s}^{\mathbf{X}} V\left(Y_{s}\right)\right] R\left(\Delta_{i}^{n}, \mathrm{~d} s\right)$ in general converges, by augmenting the Skorohod integral with $A_{i}$ and rebalancing the terms, we can identify the trace term in Theorem 6.2 more precisely.

6.1. Application of the correction formula to fractional Brownian motion. We now apply the correction formula to fractional Brownian motion with $H>\frac{1}{3}$.

THEOREM 6.3. For $1 \leq p<3$, let $Y \in \mathcal{C}^{p-\operatorname{var}}\left([0, T] ; \mathbb{R}^{d}\right)$ denote the pathlevel solution to

$$
\mathrm{d} Y_{t}=V\left(Y_{t}\right) \circ \mathrm{d} \mathbf{X}_{t}, \quad Y_{0}=y_{0} .
$$

We assume that $V \in \mathcal{C}_{b}^{k}\left(\mathbb{R}^{d} ; \mathbb{R}^{d} \otimes \mathbb{R}^{d}\right)$, with

$$
k= \begin{cases}2, & 1 \leq p<2, \\ 6, & 2 \leq p<3,\end{cases}
$$

and $\mathbf{X} \in \mathcal{C}^{0, p-\operatorname{var}}\left([0, T] ; G^{\lfloor p\rfloor}\left(\mathbb{R}^{d}\right)\right)$ is the geometric rough path constructed from the limit of the piecewise-linear approximations of fractional Brownian motion with Hurst parameter $H>\frac{1}{3}$, and covariance function

$$
R(s, t)=\frac{1}{2}\left(s^{2 H}+t^{2 H}-|t-s|^{2 H}\right) .
$$

Then almost surely, we have

$$
\begin{aligned}
\int_{0}^{T} Y_{t} \circ \mathrm{d} \mathbf{X}_{t}= & \int_{0}^{T} Y_{t} \mathrm{~d} X_{t}+H \int_{0}^{T} \operatorname{tr}\left[V\left(Y_{s}\right)\right] s^{2 H-1} \mathrm{~d} s \\
& +\int_{[0, T]^{2}} \mathbb{1}_{[0, t)}(s) \operatorname{tr}\left[J_{t \leftarrow s}^{\mathbf{X}} V\left(Y_{S}\right)-V\left(Y_{t}\right)\right] \mathrm{d} R(s, t) .
\end{aligned}
$$

PROOF. We will show that fractional Brownian motion fulfills all the requirements needed to apply Theorem 6.1 when $H>\frac{1}{3}$. Let $\rho:=\frac{1}{2 H}$ and $q:=\frac{1}{\rho} \vee 1$. The proof that $\|R\|_{\rho \text {-var; }[0, T]^{2}}<\infty$ can be found in [17]; see also [19]. Note also that $R(t)=t^{2 H}$ is of bounded variation, and thus has finite $q$-variation.

In the case $1 \leq p<2$, or $H>\frac{1}{2}$, the geometric rough path is simply $\left(1, B_{t}^{H}\right)$, and for $H \leq \frac{1}{2}$, one can invoke Theorem 2.13 to construct the geometric rough path.

Finally, it is proved in Example 1 of [19] that

$$
\|R(t, \cdot)-R(s, \cdot)\|_{\rho-\operatorname{var} ;[0, T]} \leq C|t-s|^{\frac{1}{\rho}} \quad \forall s, t \in[0, T] .
$$




\section{APPENDIX}

THEOREM 2.20. Let $\mathbf{x}=\left(1, x, \mathbf{x}^{2}\right) \in \mathcal{C}^{p-\operatorname{var}}\left([0, T] ; G^{2}\left(\mathbb{R}^{d}\right)\right)$, where $2 \leq$ $p<3$.

Let $\phi \in \mathcal{C}^{p-\operatorname{var}}\left([0, T] ; \mathcal{L}\left(\mathbb{R}^{d} ; \mathbb{R}^{e}\right)\right)$ and $\phi^{\prime} \in \mathcal{C}^{p-\operatorname{var}}\left([0, T] ; \mathcal{L}\left(\mathbb{R}^{d} ; \mathcal{L}\left(\mathbb{R}^{d} ; \mathbb{R}^{e}\right)\right)\right)$. If $\left(\phi, \phi^{\prime}\right)$ is controlled by $\mathbf{x}$, we can define the rough integral

$$
\int_{0}^{t} \phi_{r} \circ \mathrm{d} \mathbf{x}_{r}:=\lim _{\|\pi\| \rightarrow 0, \pi=\left\{0=r_{0}<\cdots<r_{n}=t\right\}} \sum_{i=0}^{n-1}\left(\phi_{r_{i}} x_{r_{i}, r_{i+1}}+\phi_{r_{i}}^{\prime} \mathbf{x}_{r_{i}, r_{i+1}}^{2}\right),
$$

where we have made use of the canonical identification $\mathcal{L}\left(\mathbb{R}^{d} ; \mathcal{L}\left(\mathbb{R}^{d} ; \mathbb{R}^{e}\right)\right) \simeq$ $\mathcal{L}\left(\mathbb{R}^{d} \otimes \mathbb{R}^{d} ; \mathbb{R}^{e}\right)$. Furthermore, denoting

$$
z_{t}:=\int_{0}^{t} \phi_{r} \circ \mathrm{d} \mathbf{x}_{r}, \quad z_{t}^{\prime}:=\phi_{t},
$$

$\left(z, z^{\prime}\right)$ is again controlled by $\mathbf{x}$, and we have the bound

$$
\|z\|_{p-\mathrm{cvar}} \leq C_{p}\|\phi\|_{p \text {-cvar }}\left(1+\|x\|_{p \text {-var; }[0, T]}+\left\|\mathbf{x}^{2}\right\|_{\frac{p}{2}-\operatorname{var} ;[0, T]}\right) .
$$

Proof. Let $0 \leq u<s<v \leq t$ and define

$$
\Xi_{u, v}:=\phi_{u} x_{u, v}+\phi_{u}^{\prime} \mathbf{x}_{u, v}^{2},
$$

which yields the defect of additivity,

$$
\left|\Xi_{u, s}+\Xi_{s, v}-\Xi_{u, v}\right| \leq\left|R_{u, s}^{\phi} x_{s, v}\right|+\left|\phi_{u, s}^{\prime} \mathbf{x}_{s, v}^{2}\right| .
$$

Now let $\theta:=\frac{3}{p}$. Then the following function

$$
\omega(u, v):=\left\|R^{\phi}\right\|_{\frac{p}{2}-\operatorname{var} ;[u, v]}^{\frac{1}{\theta}}\|x\|_{p-\operatorname{var} ;[u, v]}^{\frac{1}{\theta}}+\left\|\phi^{\prime}\right\|_{p \text {-var; }[u, v]}^{\frac{1}{\theta}}\left\|\mathbf{x}^{2}\right\|_{\frac{p}{2}-\operatorname{var} ;[u, v]}^{\frac{1}{\theta}}
$$

is a control by Lemma 2.6 as $\frac{3}{p} \geq 1$. Moreover, following the proof for Young integration (see [14]), for any partition $\pi=\left\{r_{i}\right\}$ of $[u, v]$ with $k$ subintervals, there necessarily exists some $r_{j} \in \pi$ such that

$$
\begin{aligned}
\left|\Xi_{r_{j-1}, r_{j}}+\Xi_{r_{j}, r_{j+1}}-\Xi_{r_{j-1}, r_{j+1}}\right| & \leq\left|R_{r_{j-1}, r_{j}}^{\phi} x_{r_{j}, r_{j+1}}\right|+\left|\phi_{r_{j-1}, r_{j}}^{\prime} \mathbf{x}_{r_{j}, r_{j+1}}^{2}\right| \\
& \leq 2 \omega\left(r_{j-1}, r_{j+1}\right)^{\theta} \leq 2\left(\frac{2}{k-1}\right)^{\theta} \omega(u, v)^{\theta} .
\end{aligned}
$$

Extracting $r_{j}$ leaves one with $k-1$ subintervals, and we can repeat this procedure until only $[u, v]$ remains. Since $\theta>1$, we obtain the submaximal inequality (cf. $[14,25])$

$$
\left|\int_{\pi} \phi_{r} \circ \mathrm{d} \mathbf{x}_{r}-\left(\phi_{u} x_{u, v}+\phi_{u}^{\prime} \mathbf{x}_{u, v}^{2}\right)\right| \leq C \zeta(\theta) \omega(u, v)^{\theta}
$$


where $\zeta$ is the Riemann zeta function and

$$
\int_{\pi} \phi_{r} \circ \mathrm{d} \mathbf{x}_{r}:=\sum_{i} \phi_{r_{i}} x_{r_{i}, r_{i+1}}+\phi_{r_{i}}^{\prime} \mathbf{x}_{r_{i}, r_{i+1}}^{2} .
$$

Proving (32) is equivalent to showing that

$$
\sup _{\|\pi\| \vee\left\|\pi^{\prime}\right\|<\varepsilon}\left|\int_{\pi} \phi_{r} \circ \mathrm{d} \mathbf{x}_{r}-\int_{\pi^{\prime}} \phi_{r} \circ \mathrm{d} \mathbf{x}_{r}\right| \rightarrow 0 \quad \text { as } \varepsilon \rightarrow 0,
$$

where the supremum is taken over all partitions of $[0, t]$. Without loss of generality, we can assume $\pi^{\prime}$ refines $\pi$, in which case $\|\pi\| \vee\left\|\pi^{\prime}\right\|=\|\pi\|$ and

$$
\begin{aligned}
\left|\int_{\pi} \phi_{r} \circ \mathrm{d} \mathbf{x}_{r}-\int_{\pi^{\prime}} \phi_{r} \circ \mathrm{d} \mathbf{x}_{r}\right| & =\left|\sum_{[u, v] \in \pi}\left(\phi_{u} x_{u, v}+\phi_{u}^{\prime} \mathbf{x}_{u, v}^{2}-\int_{\pi^{\prime} \cap[u, v]} \phi_{r} \circ \mathrm{d} \mathbf{x}_{r}\right)\right| \\
& \leq C_{p} \sum_{[u, v] \in \pi} \omega(u, v)^{\theta},
\end{aligned}
$$

which vanishes as $\|\pi\| \rightarrow 0$.

Continuing, we define

$$
R_{s, t}^{z}:=\int_{s}^{t} \phi_{r} \circ \mathrm{d} x_{r}-\phi_{s} x_{s, t}
$$

and using (112), we obtain

$$
\begin{aligned}
\left|z_{s, t}\right|^{p},\left|z_{s, t}^{\prime}\right|^{p} \leq & C_{p}\left(\|\phi\|_{\mathcal{V}^{p} ;[0, T]}+\left\|\phi^{\prime}\right\|_{\mathcal{V}^{p} ;[0, T]}+\left\|R^{\phi}\right\|_{\frac{p}{2}-\operatorname{var} ;[0, T]}\right)^{p} \\
& \times\left(\|x\|_{p \text {-var; }[s, t]}^{p}+\left\|\mathbf{x}^{2}\right\|_{\frac{p}{2}-\operatorname{var} ;[s, t]}^{p}\right) \\
\left|R_{s, t}^{z}\right|^{\frac{p}{2}} \leq & C_{p}\left(\left\|\phi^{\prime}\right\|_{\mathcal{V} p ;[0, T]}^{\frac{p}{2}}\left\|\mathbf{x}^{2}\right\|_{\frac{p}{2}-\operatorname{var} ;[s, t]}^{\frac{p}{2}}+\|x\|_{p-\operatorname{var} ;[0, T]}^{\frac{p}{2}}\left\|R^{\phi}\right\|_{\frac{p}{2}-\operatorname{var} ;[s, t]}^{\frac{p}{2}}\right) .
\end{aligned}
$$

From the super-additivity of the quantities on the right-hand side in the above expression, the fact that $\left(z, z^{\prime}\right)$ is controlled with norm (33) follows immediately.

Proposition 2.21. For $p \geq 2$, let

$$
\begin{aligned}
y & \in \mathcal{C}^{p-\operatorname{var}}([0, T] ; \mathcal{U}), \\
y^{\prime} & \in \mathcal{C}^{p-\operatorname{var}}\left([0, T] ; \mathcal{L}\left(\mathbb{R}^{d} ; \mathcal{U}\right)\right),
\end{aligned}
$$

and let $\phi$ be a $\mathcal{C}_{b}^{2}$ map from $\mathcal{U}$ to $\mathcal{V}$. 
Then $\phi(y) \in \mathcal{C}^{p-\operatorname{var}}([0, T] ; \mathcal{V})$ and $\nabla \phi(y) y^{\prime} \in \mathcal{C}^{p \text {-var }}\left([0, T] ; \mathcal{L}\left(\mathbb{R}^{d} ; \mathcal{V}\right)\right)$. Furthermore, if $\left(y, y^{\prime}\right)$ is controlled by $\mathbf{x} \in \mathcal{C}^{p-\operatorname{var}}\left([0, T] ; G^{2}\left(\mathbb{R}^{d}\right)\right)$, then $(\phi(y)$, $\left.\nabla \phi(y) y^{\prime}\right)$ is also controlled by $\mathbf{x}$ and we have

$$
\begin{aligned}
& \|\phi(y)\|_{p \text {-var; [0,T] }},\left\|\nabla \phi(y) y^{\prime}\right\|_{p \text {-var; [0,T] }} \\
& \quad \leq\|\phi\|_{\mathcal{C}_{b}^{2}}\|y\|_{\mathcal{V}^{p} ;[0, T]}\left(1+\left\|y^{\prime}\right\|_{\mathcal{V}^{p} ;[0, T]}\right)
\end{aligned}
$$

and

$$
\left\|R^{\phi(y)}\right\|_{\frac{p}{2}-\operatorname{var} ;[0, T]} \leq\|\phi\|_{\mathcal{C}_{b}^{2}}\left(\|y\|_{p \text {-var; }[0, T]}^{2}+\left\|R^{y}\right\|_{\frac{p}{2}-\operatorname{var} ;[0, T]}\right) .
$$

PROOF. See Lemma 7.3 in [14] for the proof in Hölder topology; the $p$ variation estimates will be derived similarly. Using the mean-value theorem, (34) can be obtained easily. To show (35) and that $\left(\phi(y), \nabla(y) y^{\prime}\right)$ is controlled by $\mathbf{x}$, we first use Taylor's theorem to obtain

$$
(\phi(y))_{s, t}=\nabla \phi\left(y_{s}\right) y_{s, t}+R_{s, t}^{\text {Taylor }}
$$

for all $s<t$ in $[0, T]$, where $\left|R_{s, t}^{\text {Taylor }}\right| \leq\|\phi\|_{\mathcal{C}_{b}^{2}}\left|y_{s, t}\right|^{2}$. From this, it follows that

$$
\left\|R^{\text {Taylor }}\right\|_{\frac{p}{2}-\operatorname{var} ;[0, T]} \leq\|\phi\|_{\mathcal{C}_{b}^{2}}\|y\|_{p \text {-var; }[s, t]}^{2} .
$$

We next use the fact that $\left(y, y^{\prime}\right)$ is controlled by $\mathbf{x}$ in equation (114), which yields

$$
(\phi(y))_{s, t}=\underbrace{\nabla \phi\left(y_{s}\right) y_{s}^{\prime}}_{=:(\phi(y))_{s}^{\prime}} x_{s, t}+\underbrace{\nabla \phi\left(y_{s}\right) R_{s, t}^{y}+R_{s, t}^{\text {Taylor }}}_{=: R_{s, t}^{\phi(y)}},
$$

and also gives

$$
\left\|R^{\phi(y)}\right\|_{\frac{p}{2}-\operatorname{var} ;[0, T]} \leq\|\nabla \phi(y)\|_{\infty}\left\|R^{y}\right\|_{\frac{p}{2}-\operatorname{var} ;[0, T]}+\left\|R^{\text {Taylor }}\right\|_{\frac{p}{2}-\operatorname{var} ;[0, T]} .
$$

Proposition 2.22 (Leibniz rule). For $p \geq 2$, let

$$
\begin{aligned}
\phi & \in \mathcal{C}^{p-\operatorname{var}}([0, T] ; \mathcal{L}(\mathcal{U} ; \mathcal{V})) \\
\phi^{\prime} & \in \mathcal{C}^{p-\operatorname{var}}\left([0, T] ; \mathcal{L}\left(\mathbb{R}^{d} ; \mathcal{L}(\mathcal{U} ; \mathcal{V})\right)\right)
\end{aligned}
$$

and we assume that $\left(\phi, \phi^{\prime}\right)$ is controlled by $\mathbf{x} \in \mathcal{C}^{p-\operatorname{var}}\left([0, T] ; G^{2}\left(\mathbb{R}^{d}\right)\right)$.

(i) Let $\psi \in \mathcal{C}^{p-\operatorname{var}}([0, T] ; \mathcal{U}), \psi^{\prime} \in \mathcal{C}^{p-\operatorname{var}}\left([0, T] ; \mathcal{L}\left(\mathbb{R}^{d} ; \mathcal{U}\right)\right)$, and suppose that $\left(\psi, \psi^{\prime}\right)$ is controlled by $\mathbf{x}$. Then the path $\phi \psi \in \mathcal{C}^{p-\operatorname{var}}([0, T] ; \mathcal{V})$ given by the composition of $\phi$ and $\psi$ is also controlled by $\mathbf{x}$, with derivative process $(\phi \psi)^{\prime}=$ $\phi^{\prime} \psi+\phi \psi^{\prime}$. In addition, we have the bound

$$
\|\phi \psi\|_{p \text {-cvar }} \leq 2\|\phi\|_{p \text {-cvar }}\|\psi\|_{p \text {-cvar }}
$$


(ii) Suppose that $\psi \in \mathcal{C}^{\frac{p}{2}-\operatorname{var}}([0, T] ; \mathcal{U})$. Then $\phi \psi \in \mathcal{C}^{p \text {-var }}([0, T] ; \mathcal{V})$ is also controlled by $\mathbf{x}$, with derivative process $(\phi \psi)^{\prime}=\phi^{\prime} \psi$. Moreover, we have the bound

$$
\|\phi \psi\|_{p-\mathrm{cvar}} \leq\|\phi\|_{p-\mathrm{cvar}}\|\psi\|_{\mathcal{V}^{\frac{p}{2} ;[0, T]}}
$$

PROOF. The statement can be seen as a corollary to the previous proposition if we consider the smooth map $\Phi(\phi, \psi)=\phi \psi$. However, we will prove it directly to get the precise bounds (36) and (37).

For the first part, it is trivial to see that $\|\phi \psi\|$ and $\left\|\phi^{\prime} \psi+\phi \psi^{\prime}\right\|_{p \text {-var; }[0, T]}$ are bounded by the right-hand side of (36). For the remainder term, we note that

$$
\begin{aligned}
(\phi \psi)_{s, t}-\left(\phi_{s}^{\prime} \psi_{s}+\phi_{s} \psi_{s}^{\prime}\right) x_{s, t} & \\
& =\phi_{t} \psi_{t}-\phi_{s} \psi_{s}-\phi_{s, t} \psi_{s}-\phi_{s} \psi_{s, t}+R_{s, t}^{\phi} \psi_{s}+\phi_{s} R_{s, t}^{\psi} \\
& =\phi_{t} \psi_{t}-\phi_{t} \psi_{s}-\phi_{s} \psi_{t}+\phi_{s} \psi_{s}+R_{s, t}^{\phi} \psi_{s}+\phi_{s} R_{s, t}^{\psi} \\
& =\phi_{s, t} \psi_{s, t}+R_{s, t}^{\phi} \psi_{s}+\phi_{s} R_{s, t}^{\psi}
\end{aligned}
$$

and thus

$$
\begin{aligned}
\left\|R^{\phi \psi}\right\|_{\frac{p}{2}-\operatorname{var} ;[0, T]} \leq & \|\phi\|_{p \text {-var; }[0, T]}\|\psi\|_{p \text {-var; }[0, T]}+\|\psi\|_{\infty}\left\|R^{\phi}\right\|_{\frac{p}{2}-\operatorname{var} ;[0, T]} \\
& +\|\phi\|_{\infty}\left\|R^{\psi}\right\|_{\frac{p}{2}-\operatorname{var} ;[0, T]} .
\end{aligned}
$$

For the second part, note that $\|\phi \psi\|$ and $\left\|\phi^{\prime} \psi\right\|_{p \text {-var; }[0, T]}$ are bounded by the righthand side of (37). Moreover, we have

$$
\begin{aligned}
(\phi \psi)_{s, t}-\left(\phi_{s}^{\prime} \psi_{s}\right) x_{s, t} & =\phi_{t} \psi_{t}-\phi_{s} \psi_{s}-\phi_{s, t} \psi_{s}+R_{s, t}^{\phi} \psi_{s} \\
& =\phi_{t} \psi_{s, t}+R_{s, t}^{\phi} \psi_{s}=: R_{s, t}^{\phi \psi}
\end{aligned}
$$

which gives

$$
\left\|R^{\phi \psi}\right\|_{\frac{p}{2}-\operatorname{var} ;[0, T]} \leq\|\phi\|_{\infty}\|\psi\|_{\frac{p}{2}-\operatorname{var} ;[0, T]}+\|\psi\|_{\infty}\left\|R^{\phi}\right\|_{\frac{p}{2}-\operatorname{var} ;[0, T]} .
$$

Acknowledgements. The authors wish to thank Samy Tindel for helpful discussion during the course of this work. Part of this work was undertaken on a trip of the first-named author to the National University of Singapore (NUS).

\section{REFERENCES}

[1] Alòs, E., Mazet, O. and Nualart, D. (2001). Stochastic calculus with respect to Gaussian processes. Ann. Probab. 29 766-801. MR1849177

[2] Bayer, C., Friz, P. and Gatheral, J. (2016). Pricing under rough volatility. Quant. Finance 16 887-904.

[3] BogacheV, V. I. (2010). Differentiable Measures and the Malliavin Calculus. Mathematical Surveys and Monographs 164. Amer. Math. Soc., Providence, RI. 
[4] Carmona, P., Coutin, L. and Montseny, G. (2003). Stochastic integration with respect to fractional Brownian motion. Ann. Inst. Henri Poincaré Probab. Stat. 39 27-68. MR1959841

[5] Cass, T., Driver, B. K. and Litterer, C. (2015). Constrained rough paths. Proc. Lond. Math. Soc. 111 1471-1518.

[6] CAss, T. and Friz, P. (2011). Malliavin calculus and rough paths. Bull. Sci. Math. $135542-$ 556.

[7] CASs, T., Friz, P. and Victoir, N. (2009). Non-degeneracy of Wiener functionals arising from rough differential equations. Trans. Amer. Math. Soc. 361 3359-3359.

[8] Cass, T., Hairer, M., LitTerer, C. and Tindel, S. (2015). Smoothness of the density for solutions to Gaussian rough differential equations. Ann. Probab. 43 188-239. MR3298472

[9] Cass, T., LitTerer, C. and Lyons, T. (2012). Rough paths on manifolds. In New Trends in Stochastic Analysis and Related Topics. A Volume in Honour of Professor K. D. Elworthy (H. Zhao and A. Truman, eds) 33-88. World Sci. Publ., Hackensack, NJ. MR2920195

[10] CAss, T., LitTerer, C. and Lyons, T. (2013). Integrability and tail estimates for Gaussian rough differential equations. Ann. Probab. 41 3026-3050. MR3112937

[11] Coutin, L. and QIAN, Z. (2002). Stochastic analysis, rough path analysis and fractional Brownian motions. Probab. Theory Related Fields 122 108-140. MR1883719

[12] Decreusefond, L. (2000). A Skorohod-Stratonovich integral for the fractional Brownian motion. In Proceedings of the 7th Workshop on Stochastic Analysis and Related Fields.

[13] Decreusefond, L. (2005). Stochastic integration with respect to Volterra processes. Ann. Inst. Henri Poincaré Probab. Stat. 41 123-149. MR2124078

[14] Friz, P. and HAIRER, M. (2014). A Course on Rough Paths. Springer, Cham.

[15] FRIZ, P. and RIEDEL, S. (2013). Integrability of (non-)linear rough differential equations and integrals. Stoch. Anal. Appl. 31 336-358. MR3021493

[16] Friz, P. and Victoir, N. (2006). A note on the notion of geometric rough paths. Probab. Theory Related Fields 136 395-416. MR2257130

[17] Friz, P. and Victoir, N. (2010). Differential equations driven by Gaussian signals. Ann. Inst. Henri Poincaré Probab. Stat. 46 369-413. MR2667703

[18] Friz, P. and Victoir, N. (2010). Multidimensional Stochastic Processes as Rough Paths: Theory and Applications, 1st ed. Cambridge Studies in Advanced Mathematics 120. Cambridge Univ. Press, Cambridge.

[19] Friz, P. and Victoir, N. (2011). A note on higher dimensional p-variation. Electron. J. Probab. 16 1880-1899. MR2842090

[20] Gubinelli, M. (2004). Controlling rough paths. J. Funct. Anal. 216 86-140. MR2091358

[21] Hairer, M. and PILLAI, N. S. (2013). Regularity of laws and ergodicity of hypoelliptic SDES driven by rough paths. Ann. Probab. 41 2544-2598. MR3112925

[22] Hu, Y., Jolis, M. and Tindel, S. (2013). On Stratonovich and Skorohod stochastic calculus for Gaussian processes. Ann. Probab. 41 1656-1693. MR3098687

[23] Inahama, Y. (2014). Malliavin differentiability of solutions of rough differential equations. J. Funct. Anal. 267 1566-1584. MR3229800

[24] Karatzas, I. and Shreve, S. (1991). Brownian Motion and Stochastic Calculus, 2nd ed. Springer, Berlin. MR1121940

[25] Lyons, T. (1998). Differential equations driven by rough signals. Rev. Mat. Iberoamericana 14 215-310. MR1654527

[26] Lyons, T. and QIAN, Z. (2003). System Control and Rough Paths. Oxford Mathematical Monographs. Oxford Univ. Press, London. MR2036784

[27] Lyons, T. J., Caruana, M. and LÉvy, T. (2007). Differential Equations Driven by Rough Paths. Lecture Notes in Math. 1908. Springer, Berlin. MR2314753 
[28] Nourdin, I., Nualart, D. and Tudor, C. A. (2010). Central and non-central limit theorems for weighted power variations of fractional Brownian motion. Ann. Inst. Henri Poincaré Probab. Stat. 46 1055-1079. MR2744886

[29] NualarT, D. (2006). The Malliavin Calculus and Related Topics, 2nd ed. Springer, Berlin. MR2200233

[30] Nualart, D. and OrTIZ-Latorre, S. (2011). Multidimensional Wick-Itô formula for Gaussian processes. In Stochastic Analysis, Stochastic Systems and Applications to Finance. World Scientific, Hackensack, NJ.

[31] Nualart, D., Rovira, C. and Tindel, S. (2003). Probabilistic models for vortex filaments based on fractional Brownian motion. Ann. Probab. 31 1862-1899. MR2016603

[32] Privault, N. (1998). Skorohod stochastic integration with respect to non-adapted processes on Wiener space. Stoch. Stoch. Rep. 65 13-39. MR1708428

[33] Revuz, D. and Yor, M. (2005). Continuous Martingales and Brownian Motion, 3rd ed. Springer, Berlin. MR1725357

[34] Samko, S. G., Kilbas, A. A. and Marichev, O. I. (1993). Fractional Integrals and Derivatives: Theory and Applications. Gordon and Breach Sci. Publishers, Yverdon.

[35] Sugita, H. (1985). On a characterization of the Sobolev spaces over an abstract Wiener space. J. Math. Kyoto Univ. 25 717-725. MR0810975

[36] Towghi, N. (2002). Multidimensional extension of L.C. Young's inequality. JIPAM. J. Inequal. Pure Appl. Math. 3 Article 22. MR1906391

DEPARTMENT OF MATHEMATICS

IMPERIAL COLLEGE LONDON

SOUTH Kensington CAMPUS

180 QUEEN'S GATE

LONDON SW7 2AZ

UNITED KINGDOM

E-MAIL: thomas.cass@imperial.ac.uk
ENGINEERING SYSTEMS AND DESIGN

Singapore University of TeChNOLOGY AND DESIGN

8 SOMAPAH ROAD

BUILDING 1, ROOM 1.502.02

SINGAPORE 487372

REPUBLIC OF SINGAPORE

E-MAIL: nengli_lim@sutd.edu.sg 